Portland State University

PDXScholar

$1-14-2020$

\title{
Applications of Triplet-Photosensitizers and Development of Photochemical Methods
}

Luke V. Lutkus

Portland State University

Follow this and additional works at: https://pdxscholar.library.pdx.edu/open_access_etds

Part of the Chemistry Commons

Let us know how access to this document benefits you.

\section{Recommended Citation}

Lutkus, Luke V., "Applications of Triplet-Photosensitizers and Development of Photochemical Methods" (2020). Dissertations and Theses. Paper 5417.

https://doi.org/10.15760/etd.7290

This Dissertation is brought to you for free and open access. It has been accepted for inclusion in Dissertations and Theses by an authorized administrator of PDXScholar. Please contact us if we can make this document more accessible: pdxscholar@pdx.edu. 
Applications of Triplet-Photosensitizers and Development of Photochemical Methods

by

Luke V. Lutkus

A dissertation submitted in partial fulfillment of the requirements for the degree of

Doctor of Philosophy

in

Chemistry

Dissertation Committee:

Theresa McCormick, Chair

Mark Woods

Rob Strongin

Erik Sanchez

Portland State University

2020 


\begin{abstract}
Photochemistry focuses on various aspects of the interaction of light with molecules. This work entails new methodology for fundamental measurements of photochemistry along with novel applications of triplet-photosenstizers. Herein described are two separate methodologies developed for the determination of the quantum yield. A method for the singlet oxygen quantum yield was developed that utilizes the reaction between singlet oxygen and dimethyl sulfoxide (DMSO) to produce dimethyl sulfone. The rate of the reaction is measured by the pressure decrease that results from the consumption of oxygen from the headspace of a sealed system. It was found that the rate of pressure decrease was directly related to the singlet oxygen quantum yield. Additionally, an alternative method for the potassium ferrioxalate actinometer was developed. $\mathrm{CO}_{2}$ production was measured two different way; by the pressure increase in a sealed system and the volume change by trapping the $\mathrm{CO}_{2}$. Both methods were considerably less laborious and showed agreeable results as compared to the traditional spectroscopic methods.
\end{abstract}

A major focus of this the thesis comprises research with photosensitizers that have significant triplet yields. Incorporating heavy atoms into chromophores typically increases the triplet yield. A predictive model for the singlet oxygen quantum yield (a lower bound triplet yield approximation) was developed for fluorescein type dyes with heavy atoms of different identities and positions. Triplet-photosensitizers were also explored for use as an aerobic catalyst and for the conversion of light to preform 
pressure-volume work. A tellurium containing triplet-photosenstizer, known as a tellurorhodamine, was used for the oxidation of thiols to disulfides. Upon irradiation the tellurium dye creates singlet oxygen and reacts with it to form a telluroxide which can act as an oxidant. Near quantitative yields were obtained for aromatic thiols; thiophenol and 2-naphthalenethiol, while aliphatic thiols resulted in degradation to the tellurorhodamine.

A reversible reaction of singlet oxygen with 1,4-dimethylnapthalene was explored for the conversion of light to pressure-volume work. Singlet oxygen, generated by a triplet-photosenstizer, was used to react with 1,4-dimethylnaphthalene to produce its corresponding endoperoxide. Over time it was shown that oxygen is consumed from the headspace of a sealed system and can result in a pressure decrease. In dark conditions, near room temperature, the endoperoxide is metastable and releases oxygen to which results in a pressure increase. 


\section{Acknowledgements}

My most sincere gratitude and appreciation is extended towards all the individuals who I

have been most fortunate to learn from over the last five years.

Specifically, I'd like to acknowledge all current and past members of the McCormick lab who have helped me so much and developed an accepting and collaborative lab culture.

I'd like to thank my family, who despite the distance, have continually found ways to show love and support throughout graduate school.

I would like to acknowledge my tortoise, Lola, for her cheerful disposition and constant belief in me.

I appreciate my graduate committee of Dr. Rob Strongin, Dr. Mark Woods, and Dr. Erik Sanchez for their valuable suggestions and insights.

Finally, I'd like to offer my most sincere gratitude to my advisor, Dr. Theresa McCormick, for her undying support and guidance over the years. 


\section{Contents}

Abstract

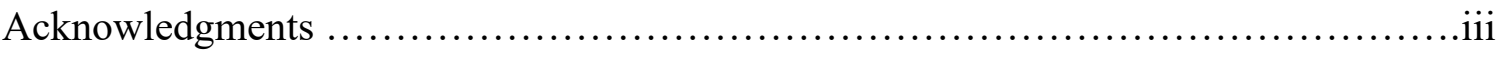

List of Tables $\quad$.................................................................

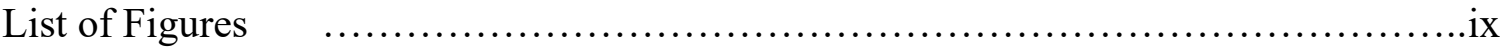

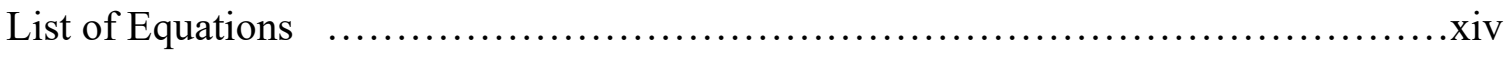

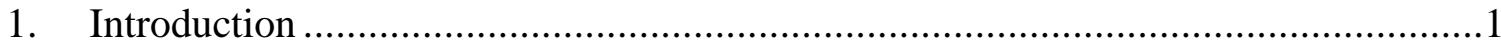

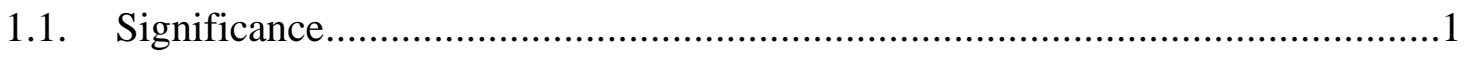

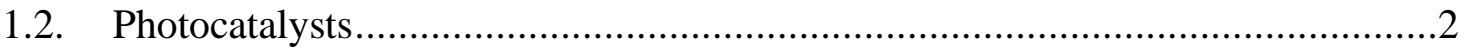

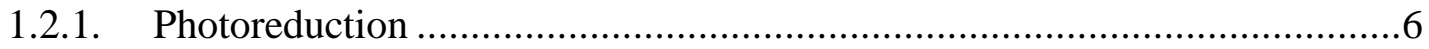

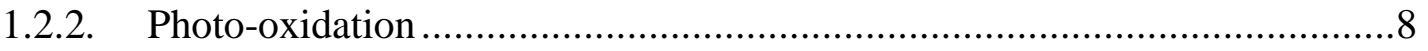

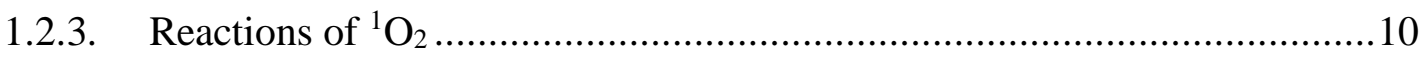

1.2.4. Reactions of ${ }^{1} \mathrm{O}_{2}$ with tellurium containing compounds............................13

1.2.5. Tellurium containing compounds as oxidants ...........................................14

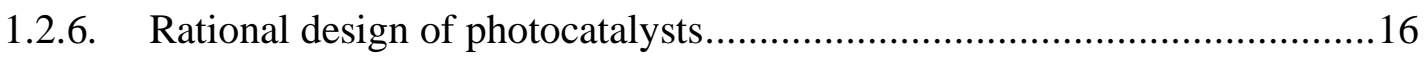

1.2.7. Methodology used to evaluate of photosensitizers ....................................17

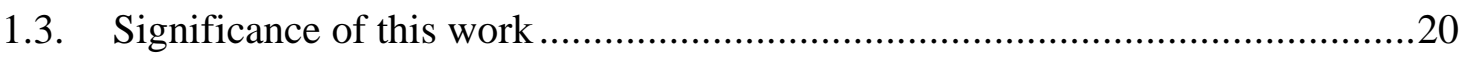

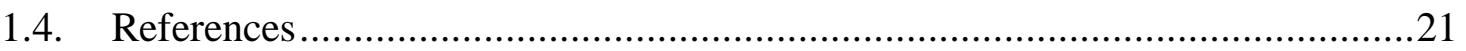

2. Quantifying Singlet Oxygen Quantum Yields by Oxygen Consumption .................26

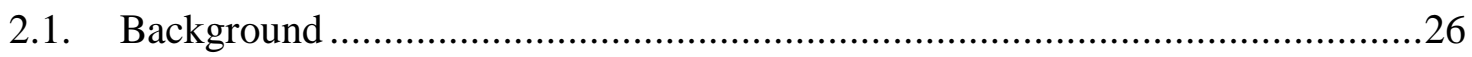

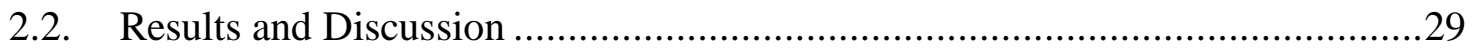

2.2.1. Oxygen consumption from Rose Bengal in DMSO ..................................30

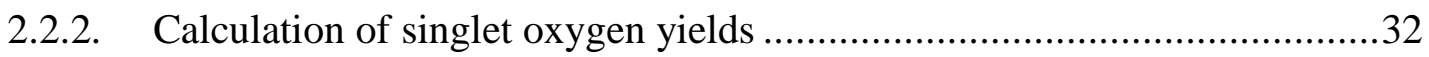

2.2.3. Experimental singlet oxygen quantum yields ..............................................34

2.2.4. Oxygen consumption in mixed solvent systems .......................................37

2.2.5. Reaction of singlet oxygen with triphenylphosphine .................................38 
2.2.6. Monitoring oxygen consumption by water displacement

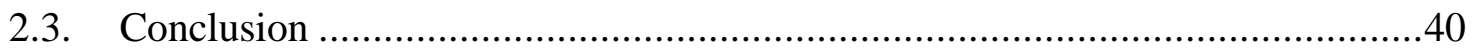

2.4. Materials and Methods ............................................................................ 41

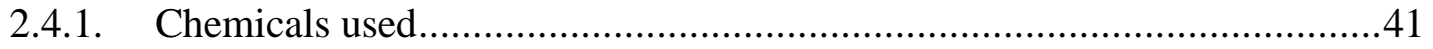

2.4.2. Oxygen consumption measurements .................................................... 41

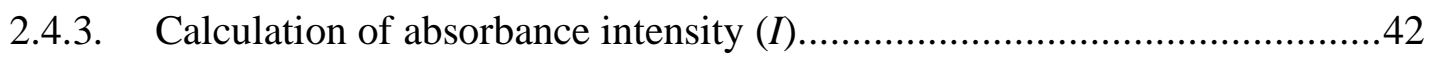

2.4.4. Water displacement method...................................................... 42

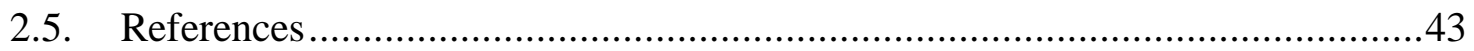

3. Photocatalytic Aerobic Oxidation Using a Tellurhodamine Chromophore ..............47

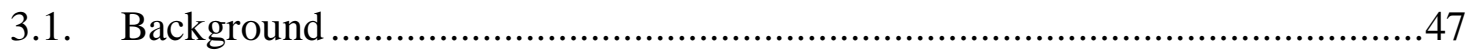

3.2. Results and Discussion .................................................................49

3.2.1. Photophysical properties of chalcogenorhodamine dyes 1 and 3 .............49

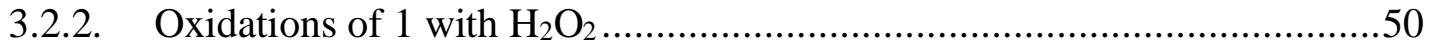

3.2.3. Photochemical reactivity of chalcogenorhodamine dyes 1 and 3 .............53

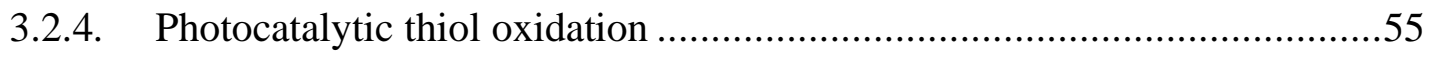

3.2.5. Reduction of telluroxide 2 with aromatic and aliphatic thiols ..................57

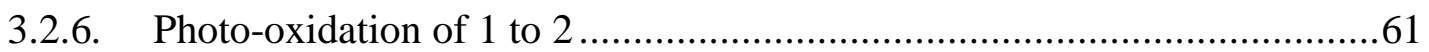

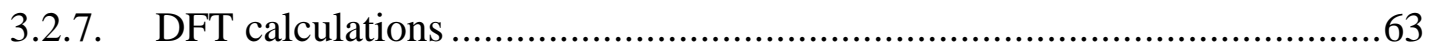

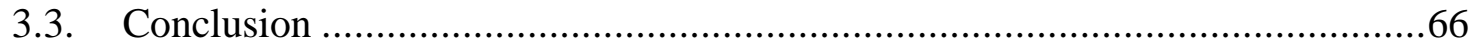

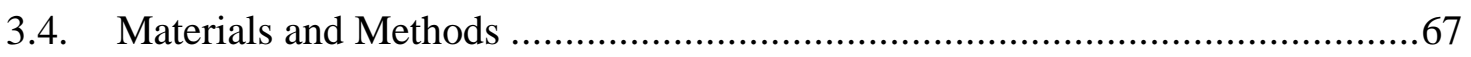

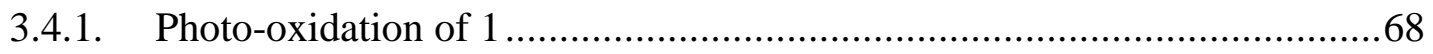

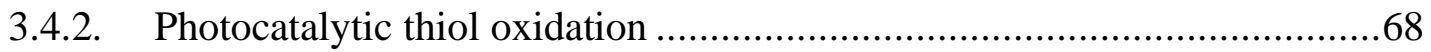

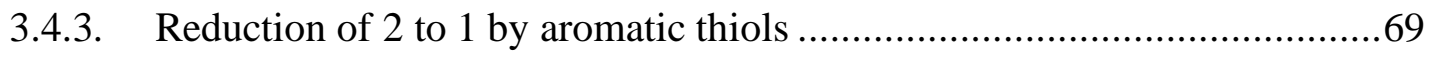

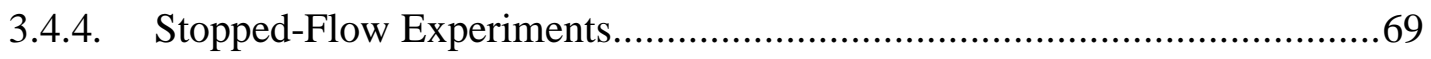

3.4.5. Determination of Singlet Oxygen Yield for Selenorhodamine..................69

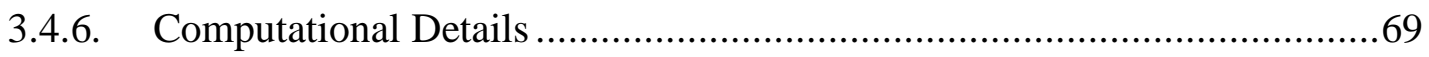

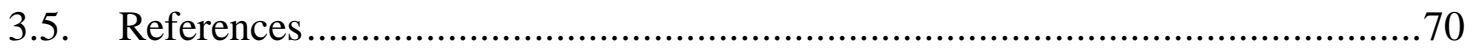

4. Simplification of the potassium ferrioxalate actinometer ................................73

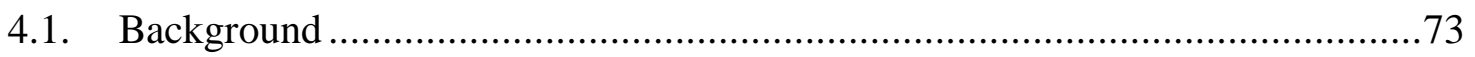




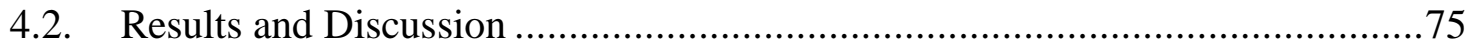

4.2.1. Traditional spectroscopic method of Fe(II) detection .............................75

4.2.2. Calculation of photons absorbed by pressure increase ...........................78

4.2.3. Calculation of photons absorbed by $\mathrm{CO}_{2}$ capture ................................83

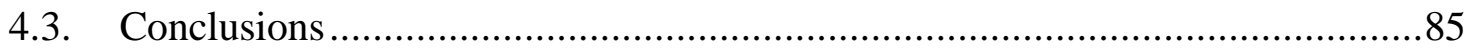

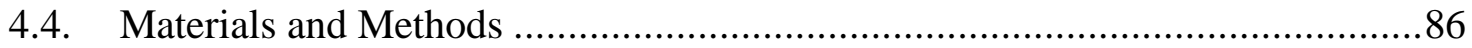

4.4.1. Synthesis of potassium ferrioxalate..................................................86

4.4.2. Traditional method determining Fe(II) concentration .............................87

4.4.3. Determination of carbon dioxide by pressure change ........................... 88

4.4.4. Determination of carbon dioxide by volume change method ...................88

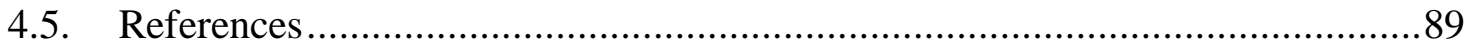

5. Predictive Modeling of Heavy Atom Effects in Halogenated Fluorescein Derivatives 91

5.1. Background ............................................................................ 91

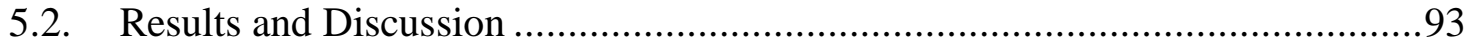

5.2.1. Computational modeling of halogenated fluorescein derivatives..............93

5.2.2. Synthesis of unreported fluorescein derivatives ................................97

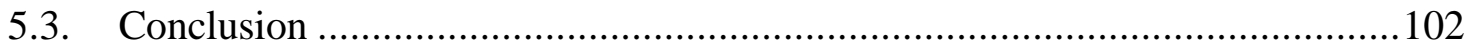

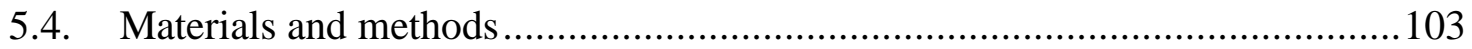

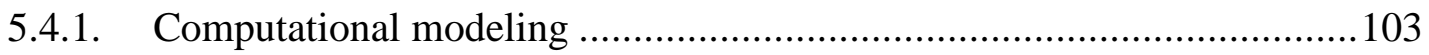

5.4.2. Synthesis of 3', 4', 5', 6' -tetrachloro fluorescein ................................... 104

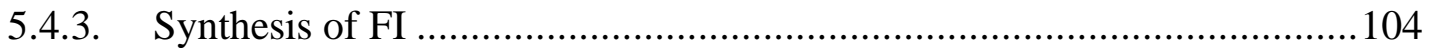

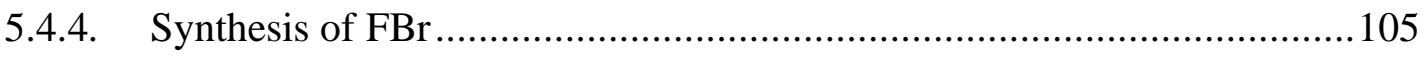

5.4.5. General methods for determination of photophysical properties .............. 105

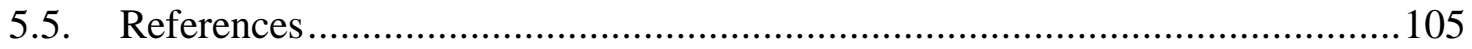

6. Conversion of Light to Mechanical Energy by Reversible Oxygen Consumption .107

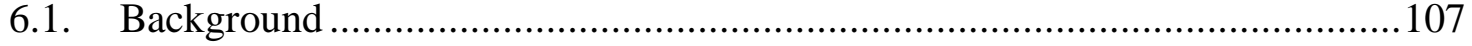

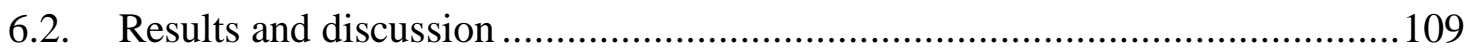

6.2.1. Pressure changes from oxygen consumption and release ...................... 109 
6.2.2. Effect of temperature on oxygen consumption and release .................... 111

6.2.3. Longevity of oxygen consumption and release....................................116

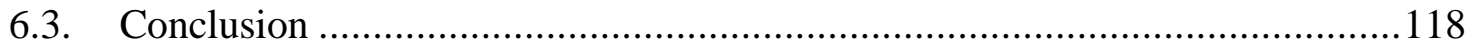

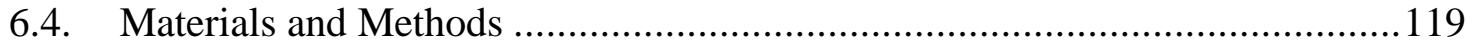

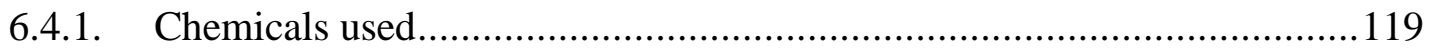

6.4.2. General set up for monitoring change of pressure over time................... 119

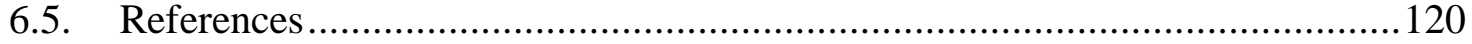

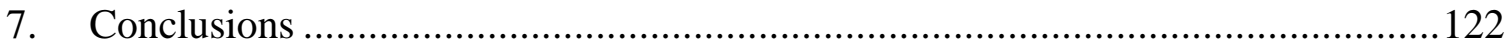




\section{List of Tables}

Table 2.1. Calculated singlet oxygen quantum yields from rates of pressure decrease ....35

Table 3.1. Oxidation of aromatic and aliphatic thiols $\left(5 \times 10^{-2} \mathrm{M}\right)$ to disulfides with 1 mol- $\% 1$ or $3\left(5 \times 10^{-4} \mathrm{M}\right)$ under aerobic conditions in $2.5 \% \mathrm{H}_{2} \mathrm{O} / \mathrm{CDCl}_{3} \ldots \ldots \ldots \ldots . . .56$

Table 4.1. Selected quantum yields of the photoreduction of potassium ferrioxalate at various wavelengths. 54

Table 5.1. Fluorescein derivatives that were computationally modeled and their reported singlet oxygen quantum yields in $\mathrm{H}_{2} \mathrm{O}$.

Table 5.2. Computationally determined heavy atom contributions from known

fluorescein derivatives.

Table 5.3. Summary of photophysical properties of FI and FBr

101 


\section{List of Figures}

Figure 1.1. Jablonksi diagram depicting selected pathways after photo-excitation ............3

Figure 1.2. Jablonksi diagram depicting possible relaxtion pathways. ..........................5

Figure 1.3. Completed Jablonski diargram....................................................

Figure 1.4. Diagram showing the valence band (VB) and conduction band $(\mathrm{CB})$ of $\mathrm{TiO} 2$ after photo-excitation to form electron (e-) hole $(\mathrm{h}+)$ pairs ................................

Figure 1.5. Simplified molecular orbital diagram of ground state oxygen and singlet oxygen

Figure 1.6. General set up for actinometry using a radiometer..................................19

Figure 2.1. Absorbance spectrum of Rose Bengal and spectrum of green and red LED intensity and absorbance at a given wavelength times the light intensity at that wavelength

Figure 2.2. A Uncorrected pressure change over time from irradiation of DMSO and triplicate solutions of DMSO containing Rose Bengal. B Adjusted pressure change of triplicate samples of Rose Bengal after subtracting the pressure change of the DMSO blank solution. C Average rate of pressure decrease over time of the triplicate samples of Rose Bengal and the linear fit.

Figure 2.3. The average pressure change over time for triplicate runs of 6 different photosensitizers irradiated by red, green, or blue LEDs in DMSO 
Figure 2.4. Pressure change over time of irradiated solutions of Rose Bengal in 50/50 $\mathrm{DMSO} / \mathrm{EtOH}$ resulting in an increase in pressure before adjusting for vapor pressure by subtracting the blank solution.

Figure 2.5. Pressure decrease over time from irradiation of Rose Bengal with triphenylphosphine in 1-butanol.

Figure 2.6. Oxygen consumption over time of an irradiated solution of Rose Bengal in DMSO measured by the water displacement method

Figure 2.7. General set up for manual method to follow oxygen consumption over time.

Figure 3.1. Oxidation of a $1 \times 10^{-5} \mathrm{M}$ solution of $\mathbf{1}$ with $\mathrm{H}_{2} \mathrm{O}_{2}$ in $10 \% \mathrm{H}_{2} \mathrm{O} / 90 \% \mathrm{MeOH}$ to give telluroxide $\mathbf{2}$ at 293 K. (a) Spectral changes for the conversion of $\mathbf{1}$ to $\mathbf{2}$ with $0.05 \mathrm{M} \mathrm{H}_{2} \mathrm{O}_{2}$. (b) Typical first-order plots for the appearance of 2 monitored at 680 $\mathrm{nm}$ at $0.01,0.03$, and $0.05 \mathrm{M} \mathrm{H} 2 \mathrm{O} 2$. (c) A plot of mean pseudo first-order rate constants from triplicate runs as a function of $\mathrm{H}_{2} \mathrm{O}_{2}$ concentration. Error bars represent \pm 1 standard deviation.

Figure 3.2. Photo-oxidation of a $1 \times 10^{-5} \mathrm{M}$ solution of $\mathbf{1}$ to give telluroxide $\mathbf{2}$ irradiated with $24 \mathrm{~W}$ white LEDs (a) in 10\% $\mathrm{H}_{2} \mathrm{O} / 90 \% \mathrm{MeOH}$ (spectrum taken every 2 min for $18 \mathrm{~min}$ ) and (b) in air-saturated $\mathrm{CDCl}_{3}$ containing $2.5 \%$ water (spectrum taken every $2 \mathrm{~min}$ for $16 \mathrm{~min})$.

Figure 3.3. ${ }^{1} \mathrm{H}$ NMR spectra following the photo-oxidation of thiophenol with 1 mol-\% of 1 under visible light irradiation in $\mathrm{CDCl}_{3}$ containing $2.5 \%$ water at $0,1 \mathrm{~h}$, and $2 \mathrm{~h}$. The thiol proton signal at $\sim 3.5 \mathrm{ppm}$ was used to monitor reaction progress. .55 
Figure 3.4. Reduction of 2 to 1 as monitored by stopped-flow spectroscopy from the diminishing of the telluroxide peak at $655 \mathrm{~nm}$ under pseudo first-order conditions a) by 2,6-dichlorothiophenol (green squares), thiophenol (red diamonds), and 2naphthalenethiol (blue triangles) and b) by 1-dodecanethiol. .58

Figure 3.5. Reduction of $2\left(1 \times 10^{-5} \mathrm{M}\right)$ to 1 using 2 equivalents of thiophenol in $\mathrm{MeOH}$.

Figure 3.6. Frontier Molecular Orbitals (FMO's) from the DFT optimized geometries of the reduced tellurorhodamine $\mathbf{1}$ and oxidized $\mathbf{2}$ of the a) LUMO and b) HOMO of $\mathbf{1}$ and the c) LUMO and d) HOMO of 2

Figure 3.7. Reaction energy diagram of the formation of $\mathbf{2}$ from the reaction of $\mathbf{1}$ with ${ }^{1} \mathrm{O}_{2}$ and $\mathrm{H}_{2} \mathrm{O}$. .64

Figure 3.8. Tellurorhodamine structures in the reaction pathway for reduction of telluroxide $\mathbf{2}$ to tellurorhodamine $\mathbf{1}$ with methanethiol. Energies are relative to $\mathbf{1}$ in

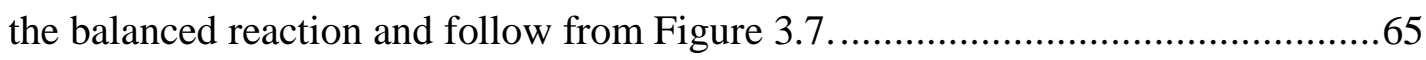

Figure 4.1. The total $\mathrm{Fe}^{2+}$ produced over time as determined spectroscopically.............77

Figure 4.2. Change in pressure over time from irradiation of potassium ferrioxalate $(0.15$

M, $10 \mathrm{~mL}$ ) with blue LEDs for first 90 minutes. .80

Figure 4.3. Change in pressure over time from the photoreduction of potassium ferrioxalate $(0.15 \mathrm{M}, 10 \mathrm{~mL})$ with blue LED irradiation over the course of 7 hours.

Figure 4.4. Depiction of $\mathrm{CO}_{2}$ capture method by water displacement. .......................84

Figure 4.5. Calculated amount of photons absorbed by the three methods. .85 
Figure 4.6. Picture of dark room set up for solution preparation. .88

Figure 5.1. Heavy atom contribution to the LUMO and triplet yield from selenium containing chromophores.

Figure 5.2. Computationally determined heavy atom contribution to the triplet LUMO compared to the literature singlet oxygen quantum yield.

Figure 5.3. Plot of the adjusted heavy atom contribution compared to the reported singlet oxygen quantum yield. .96

Figure 5.4. UV-Vis absorbance spectra of prepared dyes compared to Rose Bengal for reference.

Figure 5.5. General computationally determined heavy atom contributions to the LUMO by position.

Figure 6.1. Depiction of PV work by oxygen consumption and release using the reversible reaction of $1,4-\mathrm{DMN}$ with ${ }^{1} \mathrm{O}_{2}$.

Figure 6.2. Change in pressure over time with intermittent irradiation of $1,4-\mathrm{DMN}(0.064$ M) with Rose Bengal $\left(1 \times 10^{-5}\right)$ under atmospheric pressure at $25^{\circ} \mathrm{C}$. 111

Figure 6.3. Oxygen consumption and release at 1,4-DMN (0.064 M) with Rose Bengal (1 $\times 10^{-4} \mathrm{M}$ ) under atmospheric pressure at $20^{\circ} \mathrm{C}, 25^{\circ} \mathrm{C}, 30^{\circ} \mathrm{C}$

Figure 6.4. Rates of 1,4-DMN EP oxygen release determined by pressure increase at varying temperatures.

Figure 6.5. Continuous irradiation of Rose Bengal $\left(1 \times 10^{-4} \mathrm{M}\right)$ and 1,4-DMN $(0.064 \mathrm{M})$ under atmospheric conditions at $25^{\circ} \mathrm{C}$ until pressure decrease no longer occurs...115 
Figure 6.6. Continuous irradiation of Rose Bengal $\left(1 \times 10^{-4} \mathrm{M}\right)$ with 1,4-DMN $(0.064 \mathrm{M})$ at $25^{\circ} \mathrm{C}$ under an increased pressure of 5.3 PSI compared to atmosphere (20.2 PSI). 116

Figure 6.7. Pressure change over time of 3 cycles of irradiation Rose Bengal $\left(1 \times 10^{-4} \mathrm{M}\right)$ with 1,4-DMN $(0.064 \mathrm{M})$ for 90 minutes and 3 hours of darkness at $25^{\circ} \mathrm{C}$ under an oxygen atmosphere. 


\section{List of Equations}

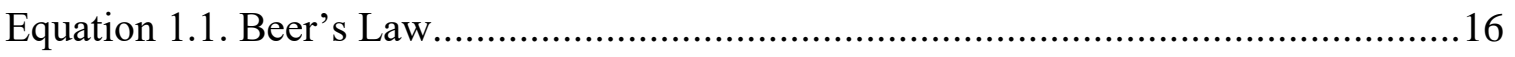

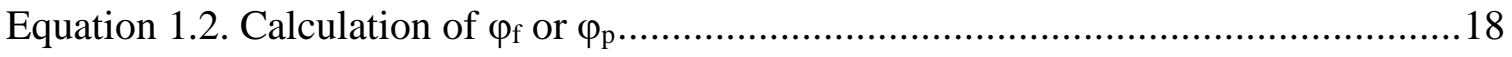

Equation 1.3. Calculation of number of photons absorbed by reference actinometer. .....20

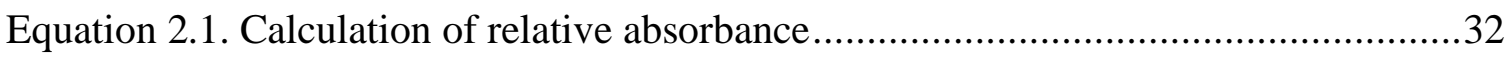

Equation 2.2. Calculation of ${ }^{1} \mathrm{O}_{2}$ quantum yield from oxygen consumption...................34

Equation 3.1. Calculation of Gibb's free energy..................................................64

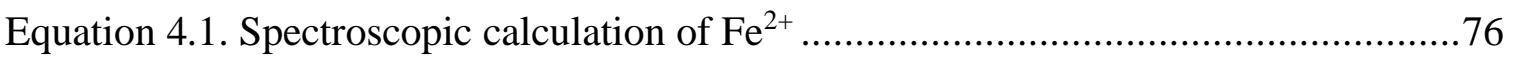

Equation 4.2. Calculation of photons absorbed ................................................. 78

Equation 4.3. Calculation of moles of $\mathrm{CO}_{2}$ by change in pressure ...........................79

Equation 4.4. Calculation of number of photons absorbed by $\mathrm{CO} 2$ production...............82

Equation 4.5. Calculation of photons per second by $\mathrm{CO} 2$ production. .........................83

Equation 5.1. Calculation of adjusted heavy atom contribution. .................................96 


\section{Introduction}

\subsection{Significance}

Traditionally, societal energy needs have been met by the combustion of hydrocarbons to produce carbon dioxide, water, and heat. Such energy pathways result in a constant demand of hydrocarbon fuel sources that are typically obtained by energy intensive mining or drilling operations. It is widely acknowledged that the carbon dioxide released from combustion is a main source of global warming. Reports from the Intergovernmental Panel on Climate Change (IPCC) have concluded that the global emissions of carbon dioxide needs to be reduced by nearly $50 \%$ to limit global warming to $1.5{ }^{\circ} \mathrm{C}$ within the next ten years. ${ }^{2}$ Currently less than $7 \%$ of global energy use comes from solar powered sources. ${ }^{3}$ Finding strategies to harness solar energy in lieu of fossil fuels is key to curbing carbon dioxide emissions.

Production of waste from chemical plants can result in more localized but devastating environmental destruction than carbon dioxide alone. Industrial level synthesis follows similar strategies to the energy industry, relying on the use of stoichiometric reagents, which need constant replenishing, resulting in wasteful byproducts. The dumping of toxic chemical waste has led to thousands of contaminated

areas across the United States know as Superfund sites. ${ }^{4-6}$ Superfund sites are large areas of uninhabitable land or bodies of water due to hazardous waste contamination that pose risks to human and environmental health alike. ${ }^{7}$ 
The energy from solar radiation striking the Earth vastly exceeds the current energy demands. To put the abundance of solar energy in perspective; converting solar irradiation at a $10 \%$ efficiency would only require capturing $0.025 \%$ of the sun's irradiation to meet energy demands. ${ }^{1}$ However, solar energy is left vastly underutilized due to the challenges of collecting and transforming light into desirable forms of energy such as electrical, thermal, and mechanical. Adopting solar driven approaches to industrial synthesis can limit the need of energy used by such plants as well as the amount of waste products. However, many challenges remain as such very few examples of industrial level photocatalysis exist. ${ }^{8}$

\subsection{Photocatalysts}

Traditionally, endothermic and nonspontaneous reactions have been driven using thermal energy. ${ }^{9}$ The abundance of solar energy makes light driven reactions an attractive alternative pathway for molecular transformations. However, challenges arise from using solar energy to drive the recombination of specific chemical bonds. For these transformations to occur a compound must first absorb the light before using the energy to achieve the desired reaction. The center of any light driven system is the photocatalyst. In bulk materials, such as silicon solar cells, light is absorbed to excite electrons from the valence band to a conduction band. However, in small molecule photocatalysts, absorbance of a photon results in excitation of an electron within discrete orbitals with many possible relaxation pathways. Ultimately, the efficacy and robustness of a photocatalyst comes down to tuning its excited state pathways for specific applications. 
The energy of the photon absorbed dictates the absorbance pathway. For most organic $\pi$ conjugated systems a Jablonski diagram can be used as a simplified visualization of the excitation and relaxation of an electron. The excitation of an electron from the highest occupied molecular orbital (HOMO) to an unoccupied orbital happens at a rate considered spontaneous. The energy of the visible light absorbed and the difference in energy between the HOMO and unoccupied molecular orbitals determines which orbital the excited electron will occupy. Excitation of an electron from a molecule in a singlet state will result in a higher energy excited singlet state with few exceptions. If the

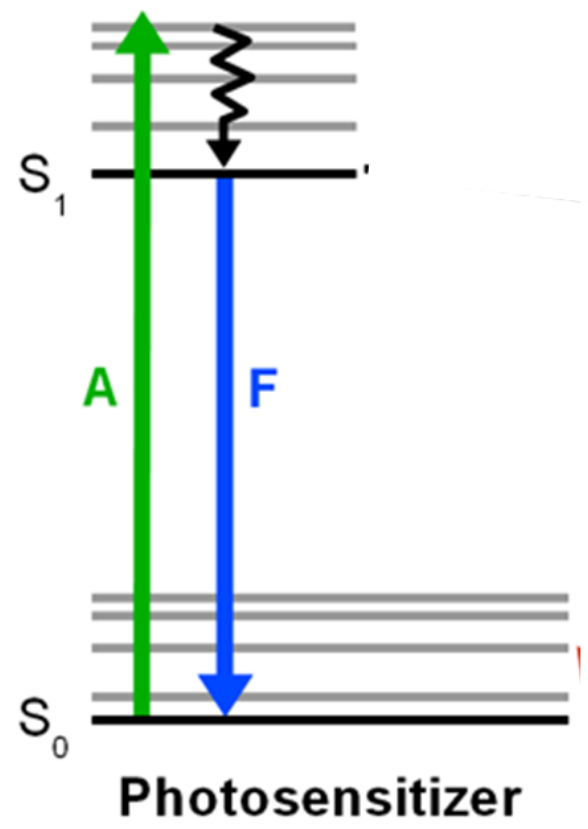

Figure 1.1. Jablonksi diagram depicting selected pathways after photo-excitation absorbance (A), fluorescence (F), and internal conversion (IC). Black lines represent electronic states and thin lines represent vibrational states. 
electron is excited to a singlet state greater than $S_{1}$ then the electron will typically relax via non- radiative pathways to $S_{1}$. The tendency of an electron to relax from $S_{n+1}$ to $S_{1}$ is known as Kasha's rule. ${ }^{10}$

An electron in an excited $S_{1}$ state can relax to the ground state by many pathways. Direct relaxation from $S_{1}$ to $S_{0}$ can happen via non-radiative decay or by radiative decay known as fluorescence. Relaxation in the form of fluorescence or non-radiative decay is relatively fast with excited state lifetimes typically spanning nanoseconds. Fluorescence may be identified from a small shift of energy in the excitation and emission known as the Stokes shift. ${ }^{10}$

An excited electron in the $S_{1}$ state may also partially relax to a lower energy state by a spin-flip to produce a molecule with two unpaired electrons known as a triplet state $\left(T_{n}\right)$. The transition of an electron from the $S_{1}$ state to a $T_{1}$ state is known as intersystem crossing (ISC). A change in the spin-state of a molecule is a forbidden transition and therefore unlikely to occur. However, ISC can be very efficient in chromophores containing heavy atoms. ${ }^{11-15}$ Vibrational pathways from the $S_{1}$ to the $T_{1}$ states can result in nearly exclusive excitation to the triplet state. Molecules with significant rates of intersystem crossing are known as triplet-photosensitizers and can excite other molecules in triplet states. For this reason triplet-photosensitizers are often sensitive to ground state oxygen which is in a triplet spin-state. Energy transfer from an excited $T_{1}$ state to oxygen results in the relaxation of the donor molecule to the $\mathrm{S}_{0}$ ground state and an excited singlet state of oxygen. ${ }^{16}$ 


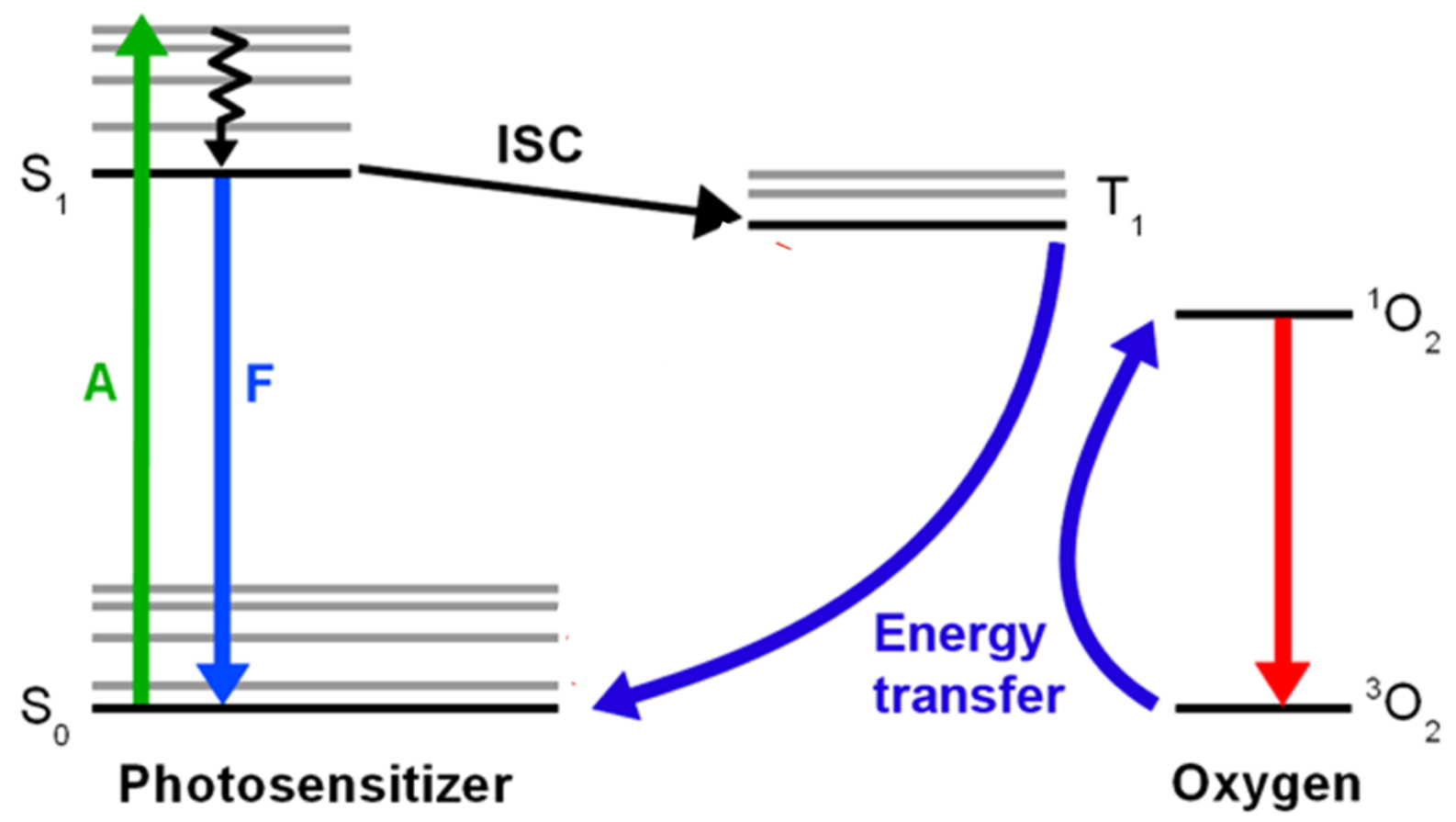

Figure 1.2. Jablonksi diagram depicting possible relaxtion pathways including intersystem crossing (ISC) from an excited $\mathrm{S}_{1}$ to a $\mathrm{T}_{1}$ state and energy transfer to ${ }^{3} \mathrm{O}_{2}$ to produce ${ }^{1} \mathrm{O}_{2}$.

Other relaxation pathways from the $T_{1}$ state also exist. To relax back to $S_{0}$ the electron must undergo a spin flip once again. The electron may relax via non-radiative pathways or radiatively, known as phosphorescence. Phosphorescence can be distinguished from fluorescence by a large Stokes shift or by a long-lived excited lifetime typically greater than microseconds. ${ }^{10}$ The long lifetime of excited triplet states typically make them more attractive photocatalysts because of the time needed for reactants to diffuse within the vicinity of the photocatalyst before relaxation occurs. ${ }^{17}$

Molecules rarely undergo relaxation via only one specific pathway. Therefore, determining the relative probability of relaxation via each possible pathway in a molecule 
can help create a more complete picture of the molecule's excited states. The relative likelihood of each relaxation pathway can be taken as a fraction from the absorbance of one photon which is referred to as the quantum yield. The quantum yield of a specific pathway can encompass transitions of multiple pathways. For example, the quantum yield of ISC would be the sum of both the phosphorescence quantum yield and nonradiative $T_{1}$ to $S_{0}$ transition.

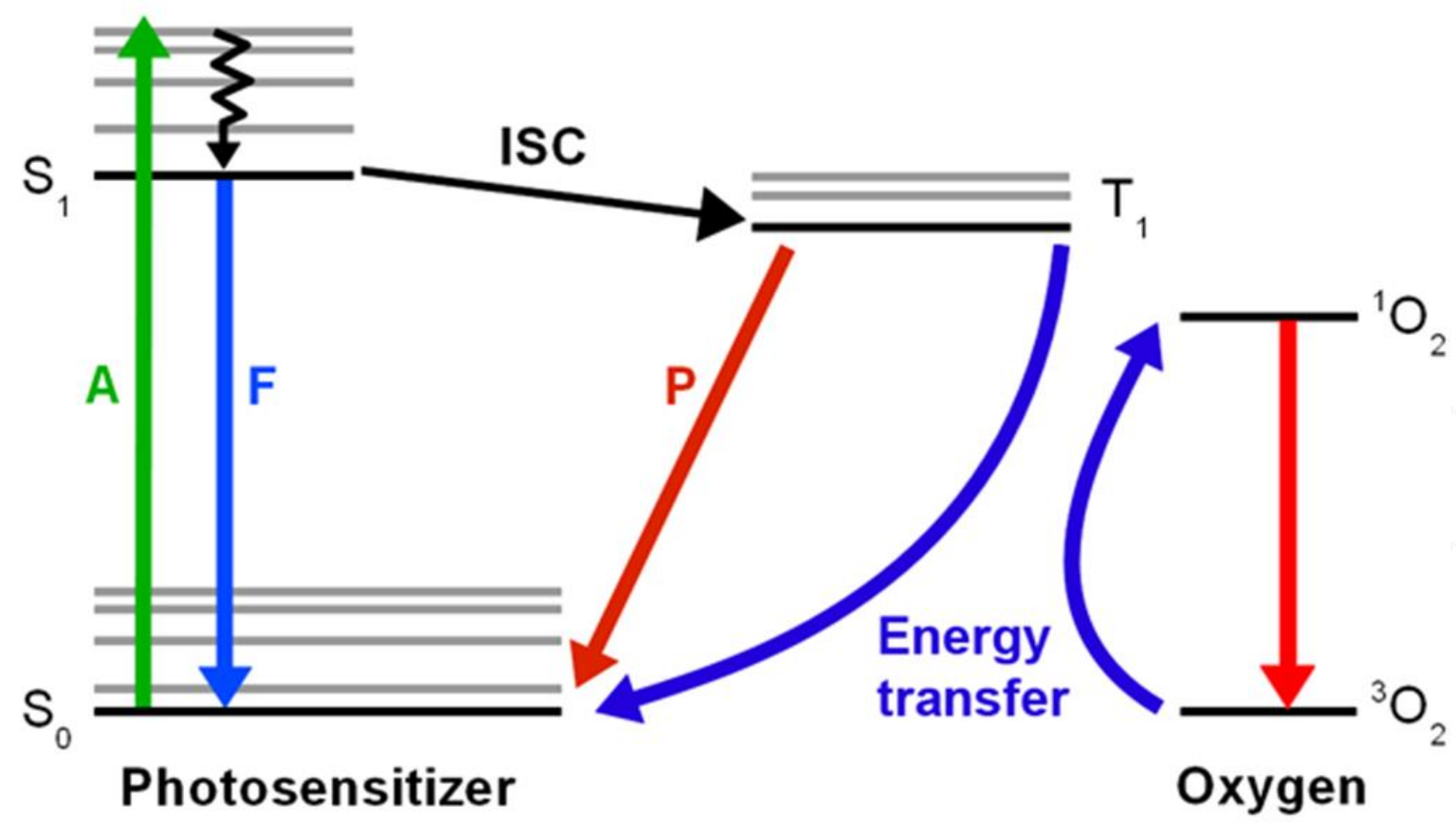

Figure 1.3. Completed Jablonski diargram depicting the most common relaxation pathways after photoexcitation including phosphorescence $(\mathrm{P})$ and non-radiative decay (NR) from the $\mathrm{T}_{1}$ state.

\subsubsection{Photoreduction}


Light driven reduction has been demonstrated both on intramolecular and intermolecular level. ${ }^{18-22}$ Intramolecularly, photoreduction occurs when absorption results in the release of an oxidative leaving group. For intramolecular photoreduction to occur an electron must be excited to an antibonding orbital. The excitation of an electron to an antibonding orbital can results in subsequent dark reactions which result in reduction of the chromophore. For example, tellurophenes have been shown to undergo photoreductive elimination of halogens from the tellurium center. ${ }^{20,23,24}$ Upon excitation of an electron to the antibonding orbital of the tellurium - bromine bond a $\mathrm{Br}$ atom is released. The tellurophene then releases the second bromine in a dark reaction as shown in Scheme 1.1.

Scheme 1.1. Photoreduction of $\mathrm{Br}_{2}$ from a tellurophene chromophore. ${ }^{24}$<smiles>[R]C1=CC=C([R])[Te]1Br</smiles>

Intermolecular photoreduction requires multiple species to either accept or donate electrons. The excitation of an electron in a photocatalyst to the LUMO must be higher in energy than the LUMO of the electron acceptor. Upon electron transfer from the photocatalyst an electron donor is often employed to donate an electron to regenerate the

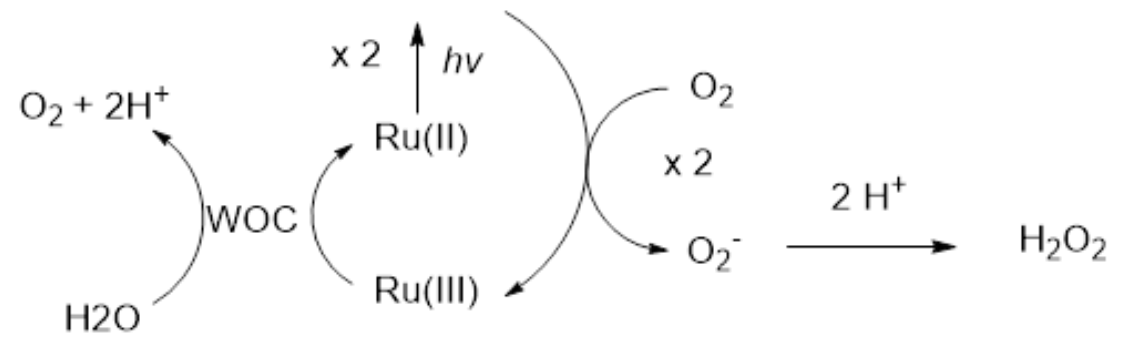

Scheme 1.2. Photoreduction of oxygen to produce hydrogen peroxide. ${ }^{25}$ 
photocatalyst. The HOMO of the electron donor must be greater in energy than the HOMO of the oxidized photocatalyst for electron transfer to occur. For example, in the photoreduction of dioxygen to hydrogen peroxide, a ruthenium chromophore $(\mathrm{Ru})$ was used for electron transfer to oxygen (Scheme 1.2). ${ }^{25}$ Upon transferring two electrons to reduce oxygen, it may be protonated to form hydrogen peroxide. The reduction of oxygen results in the oxidation of $\mathrm{Ru}$ (II) to $\mathrm{Ru}$ (III). A water oxidation catalyst (WOC) was used as the electron donor to regenerate $\mathrm{Ru}(\mathrm{III})$ back to $\mathrm{Ru}(\mathrm{II})$ making the system catalytic.

\subsubsection{Photo-oxidation}

Light driven oxidation reactions are of great importance for the degradation of

organic compounds in water and air purification. ${ }^{26-28}$ Titanium dioxide $\left(\mathrm{TiO}_{2}\right)$ has been reported for numerous photo-oxidations. $\mathrm{TiO}_{2}$ may be used heterogeneously in aqueous solutions for the photodecomposition of organic compounds. Irradiation of $\mathrm{TiO}_{2}$ with UV or near UV light has been shown to oxidize a number of organic compounds. As a heterogeneous system the $\mathrm{TiO}_{2}$ can be recovered and has shown great catalytic stability over a number of repeated cycles. 


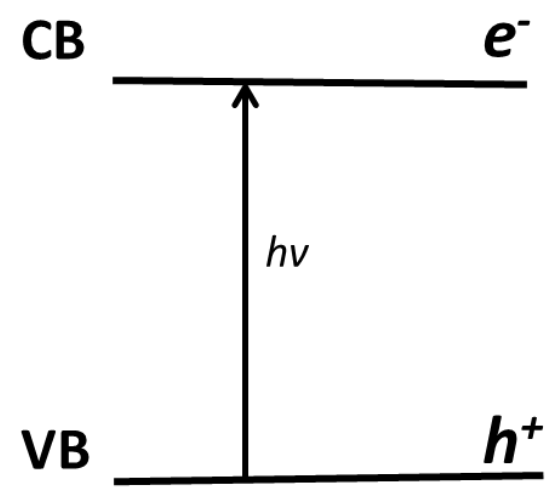

Figure 1.4. Diagram showing the valence band (VB) and conduction band (CB) of $\mathrm{TiO}_{2}$ after photo-excitation to form electron $\left(\mathrm{e}^{-}\right)$hole $\left(\mathrm{h}^{+}\right)$pairs. ${ }^{29}$

The photocatalytic properties of $\mathrm{TiO}_{2}$ and other metal oxides are often viewed as the result of charge separation from the excitation of an electron to the conduction band of the catalyst. This excitation results in electron-hole pairs in which the valence band because positively charged as a result of the electron being excited to the conduction band. This separation of charges can be used for the oxidation of organic pollutants to produce $\mathrm{CO}_{2}$ and $\mathrm{H}_{2} \mathrm{O}$. The rate of photo-oxidations of organics using $\mathrm{TiO}_{2}$ is often slowed in anhydrous conditions. ${ }^{26}$ Water can aid the rate of oxidation by the formation of hydroxides on the surface of $\mathrm{TiO}_{2}$ which can be oxidized to the more reactive hydroxyl radical (Scheme 1.3).

Scheme 1.3. Formation of hydroxyl radical from $\mathrm{TiO}_{2}{ }^{29}$

$$
\mathrm{OH}^{-}+h^{+} \longrightarrow \dot{\mathrm{OH}}
$$

While $\mathrm{TiO}_{2}$ can be used for a number of photo-oxidations, it is often too harsh of a photocatalyst for selective functional group transformations. The necessity of UV light 
for photoexcitation also hinders its uses. Another approach to photo-oxidation is the use of triplet photosensitizers to create singlet oxygen $\left({ }^{1} \mathrm{O}_{2}\right) .{ }^{1} \mathrm{O}_{2}$ has been used a lone oxidant and can be created by triplet photosensitizers over a broad spectrum of excitation wavelengths. ${ }^{16}$

\subsubsection{Reactions of ${ }^{1} \mathrm{O}_{2}$}

Excitation of triplet photosenstizers in the presence of oxygen can transfer energy from the excited-triplet state to give rise to the more reactive form of ${ }^{1} \mathrm{O}_{2} \cdot{ }^{30}{ }^{1} \mathrm{O}_{2}$ is typically a stronger oxidant than ${ }^{3} \mathrm{O}_{2}$ because it is higher in energy. The rate of oxidation by ${ }^{1} \mathrm{O}_{2}$ is typically kinetically faster than ${ }^{3} \mathrm{O}_{2}$ because most organic molecules are in a singlet ground state which reacts more readily with other singlet states due to the conservation of spin. Using ${ }^{1} \mathrm{O}_{2}$ as an oxidant is economically and environmentally advantageous because it can be formed from atmospheric oxygen. As an excited state of oxygen, ${ }^{1} \mathrm{O}_{2}$ relaxes to back to ${ }^{3} \mathrm{O}_{2}$ and therefore creates no waste products if excess is produced. ${ }^{1} \mathrm{O}_{2}$ has been used for a wide variety of organic transformations as shown in Scheme 1.4. 


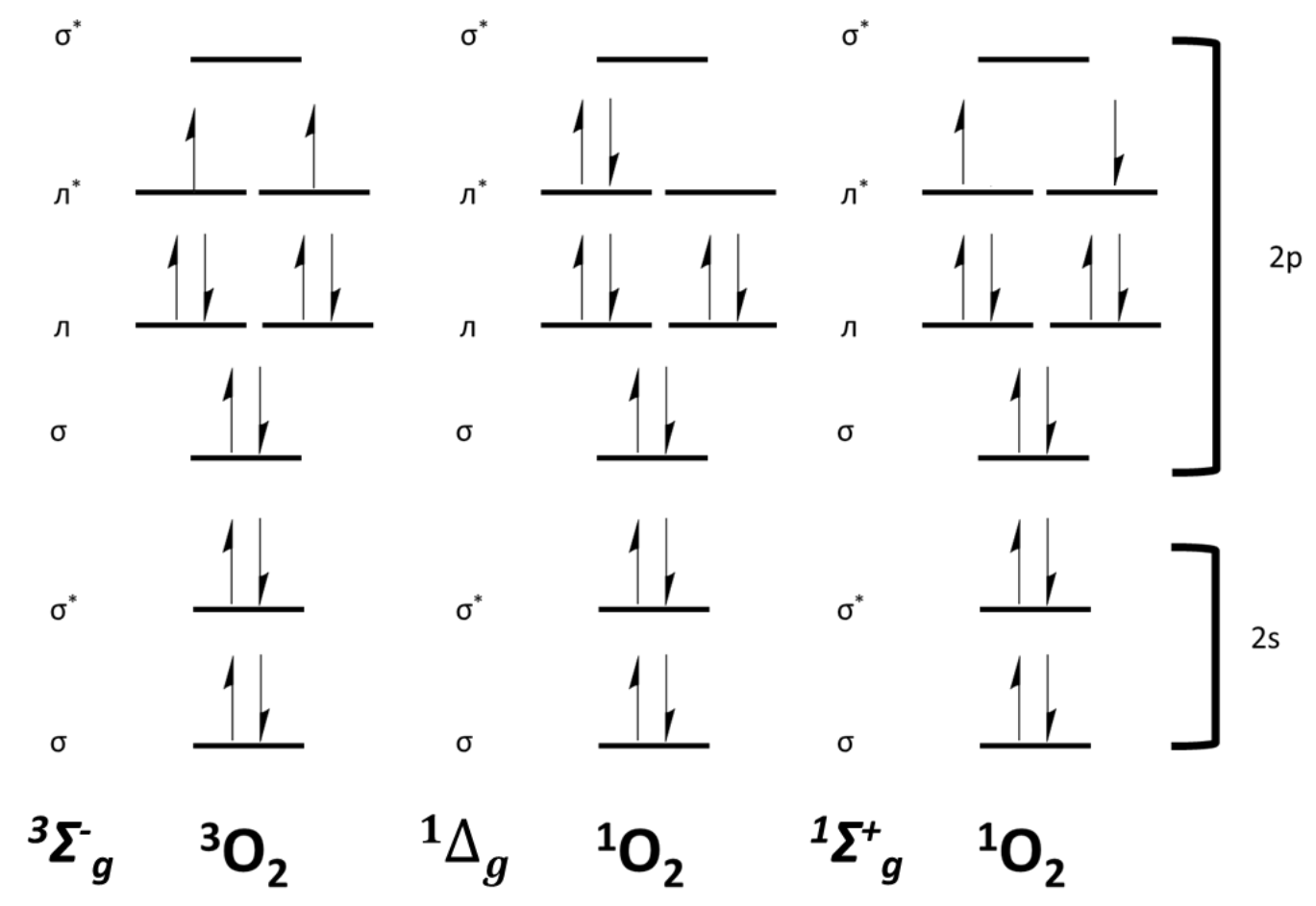

Figure 1.5. Simplified molecular orbital diagram of ground state oxygen $\left({ }^{3} \mathrm{O}_{2}\right.$, left $)$ and singlet oxygen $\left({ }^{1} \mathrm{O}_{2}\right.$, middle and right $) .{ }^{55}$ Note ${ }^{1} \Sigma^{+} g$ is considered a transient species and not expected to participate in reactions. 

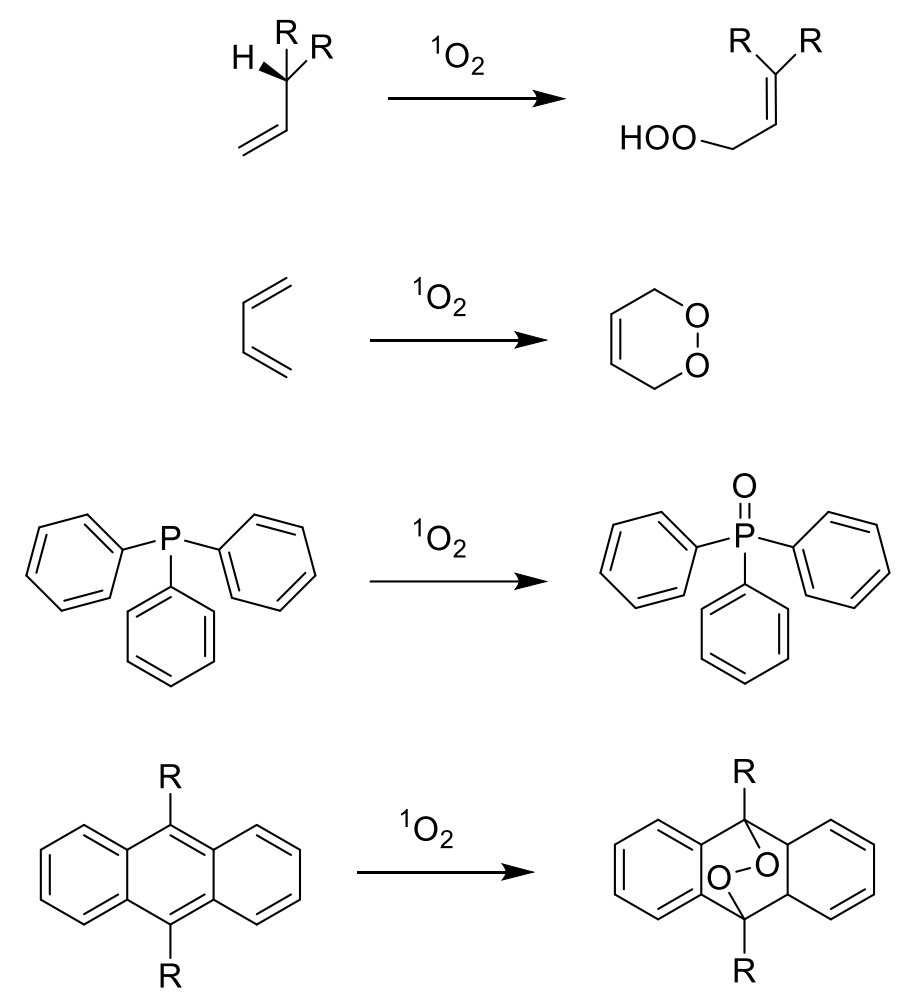

Scheme 1.4. Selected examples of ${ }^{1} \mathrm{O}_{2}$ reactions.

${ }^{1} \mathrm{O}_{2}$ can react with alkenes to produce hydroperoxides. ${ }^{31}$ In the case of dienes, ${ }^{1} \mathrm{O}_{2}$ can react in a $4+2$ fashion to form an endoperoxide. ${ }^{32-37}$ Endoperoxides can be stable at room temperature or reversible depending on the substituents. In anthracene endoperoxides the stability is typically dictated by the identity of the R groups at the 9 and 10 positions. ${ }^{32,38}{ }^{1} \mathrm{O}_{2}$ can also react with heteroatoms such as phosphorous in triphenylphosphine to produce its corresponding oxide. ${ }^{39}$

While ${ }^{1} \mathrm{O}_{2}$ may offer a cheap and green method for the oxidation of many compounds it does have its drawbacks. The lifetime of ${ }^{1} \mathrm{O}_{2}$ is solvent dependent but can be very short, for example, in water it has a half-life of $20 \mu \mathrm{s} .{ }^{30}$ Furthermore, ${ }^{1} \mathrm{O}_{2}$ often 
struggles with selective oxidations. This can be caused in part from the one electron oxidation of a substrate by ${ }^{1} \mathrm{O}_{2}$ to produce the superoxide anion. To alleviate these challenges, ${ }^{1} \mathrm{O}_{2}$ has been used in conjunction with tellurium containing compounds which may be oxidized by ${ }^{1} \mathrm{O}_{2}$ to produce $\mathrm{Te}(\mathrm{IV})$ or $\mathrm{Te}(\mathrm{VI})$ species which can be used as an oxidant. $^{40-42}$

\subsubsection{Reactions of ${ }^{1} \mathrm{O}_{2}$ with tellurium containing compounds}

Many different compounds containing tellurium have been reported to react with

${ }^{1} \mathrm{O}_{2}$. A variety of oxidized species have been proposed and are dependent on the organic framework of the molecule. Solvent effects, most notably the difference between protic and aprotic solvents, can also play a role in determining the predominate oxidized species. ${ }^{42}$ While many oxidized species have been determined using x-ray crystallography, a wide range of oxidized structures have been proposed to fit experimental data. Examples of proposed and isolated oxidized structures are shown below in Scheme 1.5.

The proposed and isolated oxidized structures show that sterics play a major role in determining the oxidized species. Julolidine groups favor telluroxide formation ( $\mathrm{TeOx}-$ 1) by sterically hindering the formation of oligomers. Rhodamine and phenyl groups offer less steric hindrance and can form oxygen bridged oligomers (TeOx-3) or dihydroxy species (TeOx-2). Mesityl-substituted aryl groups at the 4-position favor an orthogonal confirmation resulting in steric protection from the oxygen bridging between carbon and tellurium compared to unprotected groups such as TeOx-4. Less sterically 
hindered tellurium compounds can also result in a Te(VI) oxidation state as an oligomer $(\mathrm{TeOx}-4)$ or a monomer (TeOx-5).

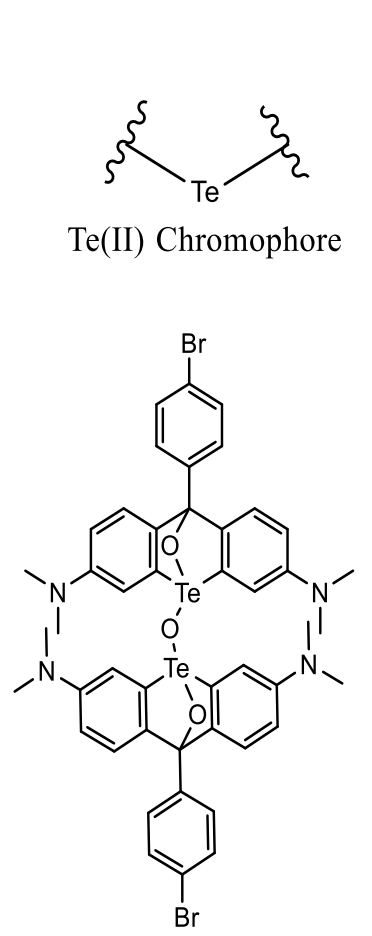

TeOx-3 $3^{a, 41}$

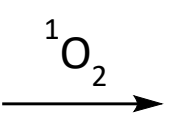<smiles></smiles>

TeOx-1 $\mathbf{1}^{a, 56}$

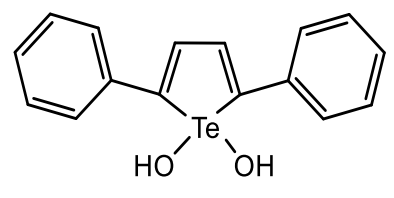

TeOx-2 $2^{57}$

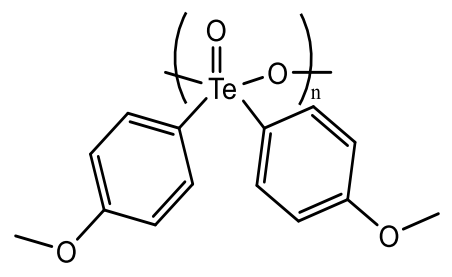

TeOx-4 4<smiles>CC(C)c1cc(C(C)C)c([PH](=O)(=O)c2c(C(C)C)cc(C(C)C)cc2C(C)C)c(C(C)C)c1</smiles>

TeOx-5 $5^{a, 42}$

Scheme 1.5. Proposed and isolated oxidized structures of different tellurium

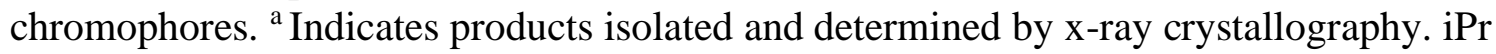
denotes isopropyl groups. Oxidations occurred in various solvents all with water present.

\subsubsection{Tellurium containing compounds as oxidants}

Tellurium containing compounds have been shown to increase rate of thiol

oxidation to disulfides with hydrogen peroxide. ${ }^{43}$ More recently tellurium chromophores oxidized by ${ }^{1} \mathrm{O}_{2}$ produced by photosensitizers have been shown to catalytically oxidize 
thiols. ${ }^{44}$ The oxidized compound $\mathbf{1 3}$ was proposed because the oxidized species was shown to oxidize 4 equivalents of thiols indicating 2 oxygen atoms per chromophore. In both cases the oxidation of thiols involve the initial oxidation of $\mathrm{Te}$ (II) to $\mathrm{Te}$ (IV) or $\mathrm{Te}(\mathrm{VI})$ which is then reduced by thiols to produce the corresponding disulfides and water.

Oxidized Te(IV) species such as a telluroxide have been shown to behave in both oxygenase and oxidase like chemistry. An oxygenase type of reaction involves the transfer of an oxygen atom to a substrate while oxidase like behavior means the oxidation by removal of electrons from a substrate. Telluroxides have been used in an oxygenase type manner for heteroatom oxidation. Phosphines and phosphine ethers have been oxidized to their corresponding oxides by telluroxides. ${ }^{45}$ Thiol oxidation to disulfides using telluroxides has been reported and is a oxidase type of reaction. Formation of telluroxides has been reported from a variety of co-oxidants such as hydrogen peroxide, sodium hypochlorite, meta-chloroperoxybenzoic acid, and ${ }^{1} \mathrm{O}_{2} \cdot{ }^{46}$

To produce telluroxides from ${ }^{1} \mathrm{O}_{2}$ typically an auxiliary photosensitizer is required. ${ }^{42}$ However, incorporating tellurium as the heteroatom into the framework of a chromophore has resulted in self-sensitized tellurium containing chromophores capable of producing ${ }^{1} \mathrm{O}_{2}$ when irradiated. Tellurium containing chromophores are typically good triplet-photosensitizers because of the incorporation of the heavy tellurium atom. Addition of heavy atoms to a chromophore typically results in a red shifted absorption and increased intersystem crossing yields. ${ }^{47}$ Tellurium containing rhodamine dyes show similar trends as near IR absorbers with high singlet oxygen yields. ${ }^{41,48}$ 


\subsubsection{Rational design of photocatalysts}

There are a number of considerations of how to design or choose a photocatalyst for a system. However, there are key characteristics that are sought after for most photocatalysts. The excitation wavelength of the photocatalyst is vital to consider and depends on the irradiation source. For solar excitation, it is ideal to have a photocatalyst that can be excited using red-NIR light because it is abundant in the solar spectrum. ${ }^{48,49}$ Excitation by UV light is challenging because of its high energy it may result in the degradation of organic compounds. However, the absorbance wavelength of a chromophore is dependent on the HOMO-LUMO energy gap and therefore certain systems may require higher energy excitation for specific transformations.

The molar absorptivity of a molecule is used as a way to compare how much light is being absorbed at a certain wavelength. Absorbance is determined using Beer's Law from Equation 1.1. A high molar absorptivity is ideal for the efficient absorption of as much light as possible with a minimum amount of photocatalyst.

Equation 1.1. Beer's Law

$$
A=\varepsilon l c
$$

As shown in Equation 1.1 the absorbance $(A)$ is equal to the molar absorptivity $(\varepsilon)$ multiplied by the path length $(l)$ and concentration of the species absorbing $(c)$. While it can be seen that increasing the path length can increase the absorbance this is often unfavorable for the same reasons as increasing the concentration. The path length refers to the volume that the sample that the light must pass through. Therefore increasing the 
path length will often require increasing the amount of photosensitizer used and amount of solvent.

After absorption of light, the excited state pathways of the photocatalyst determine its efficiency. Therefore, a high molar absorptivity alone does not always result in an efficient photocatalyst. The results of the excited state pathways of chromophores are often reported as quantum yields $(\phi)$. The quantum yield can be used to describe the likelihood of a photocatalytic process occurring per photon absorbed or the excited state relaxation pathways of a chromophore. ${ }^{50}$ For example, the fluorescence quantum yield $\left(\phi_{f}\right)$ is the amount of photons emitted through fluorescence per every photon absorbed. The $\phi$ of a light driven reaction is determined by the moles of product per mole of photons absorbed. A wide variety of techniques have been used for the determination of different types of quantum yields.

\subsubsection{Methodology used to evaluate of photosensitizers}

To determine the $\phi_{\mathrm{f}}$ or phosphorescence quantum yield $\left(\phi_{\mathrm{p}}\right)$ the amount of photons absorbed and emitted must be determined. Typically, these quantum yields are determined by comparing their photoluminescence to a known standard, however, integration spheres may be used which do not require reference samples. Using a fluorimeter or phosphorimeter after measuring the samples absorbance at the excitation wavelength the $\phi_{\mathrm{f}}$ or $\phi_{\mathrm{p}}$ may be determined using Equation 1.2, comparing to a known reference. $^{10}$ 
Equation 1.2. Calculation of $\phi_{f}$ or $\phi_{p}$

$$
\varphi_{\text {sample }}=\frac{E_{\text {sample }}}{A_{\text {sample }}} \times \frac{E_{\text {reference }}}{A_{\text {reference }}} \times \varphi_{\text {reference }}
$$

Where $E$ is the emission integral of the sample or reference and the $A$ is the absorbance at the excitation wavelength. The sample and the reference should be excited at the same wavelength to overcome the different light intensity of the excitation source. Determining the $\phi$ of other processes that do not emit light require different techniques such as transient absorbance spectroscopy or indirect methods that measure the amount of product from a light driven reaction. ${ }^{51}$

The triplet quantum yield $\left(\phi_{\mathrm{t}}\right)$ refers to the amount of triplet states produced per photons absorbed. While the relaxation from a triplet state may result in phosphorescence, there are additional relaxation pathways that are not emissive and must be accounted for to determine the $\phi_{\mathrm{t}}$. A common lower bound approximation to the $\phi_{\mathrm{t}}$ is the singlet oxygen quantum yield $\left(\phi_{\Delta}\right)$ because ${ }^{1} \mathrm{O}_{2}$ is created from quenching excited triplet-states. The phosphorescence of ${ }^{1} \mathrm{O}_{2}$ can be used to determine the $\phi_{\Delta}$ by comparing the emission integral to a known standard as in Equation 1.2. ${ }^{52}$

To determine the $\phi$ of a light driven reaction actinometry is needed to determine the amount of photons absorbed. Actinometry is a technique used to measure the amount of energy given off from a light source. This may be accomplished either using a relative method to a light driven reaction with a known $\phi$ or an absolute method using a radiometer to measure the amount of photons absorbed. ${ }^{53}$ 


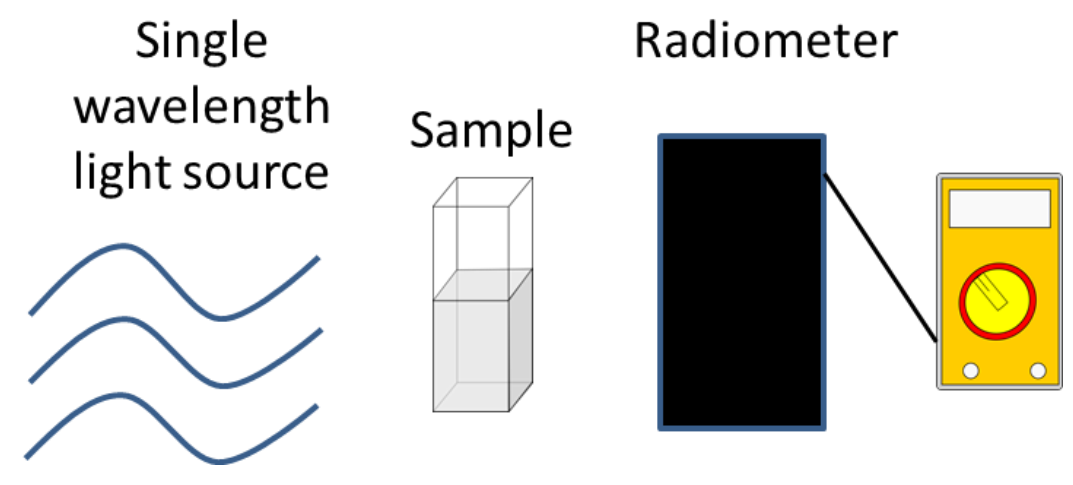

Figure 1.6. General set up for actinometry using a radiometer.

Using a radiometer to measure the amount of light without the sample and then repeating the measurement with the sample in place; the amount of photons absorbed can be determined by the difference. Then a method is required to determine the moles of product produced at a certain time point. The quantification of the moles of product differs depending on the reaction, however, the sample must be kept free from incident light prior to the measurement to properly quantify only the photons from the intended light source. A monochromatic light source must be used because the calculation of the amount of photons is based on the energy measured by the radiometer. Differing wavelengths of light result in different energy as calculated by the Planck-Einstein relationship.

A variety of chemical actinometers with known $\phi$ already exists and may be used for reference to determine the $\phi$ of an unknown light driven reaction. ${ }^{54}$ The selection of a reference chemical actinometer depends on the excitation wavelength being used. The general calculation for the amount of photons absorbed by the reference actinometer may 
be calculated using Equation 3. Dividing the moles of product formed from the unknown light driven reaction by the number photons can then afford the $\phi$.

Equation 1.3. Calculation of number of photons absorbed by reference actinometer.

$$
\boldsymbol{n}_{\text {photons }}=\frac{\text { molecules of product }}{\varphi_{\lambda}}
$$

\subsection{Significance of this work}

Absorption of light by a chromophore results in an excited-state with various relaxation pathways. The development of compounds that can absorb light energy to initiate reactions is vital for the transition to sustainable industrial processes for chemical transformation and solar energy storage. The key to improving these processes lies in the selection or design of the proper photocatalyst. Tuning the photocatalysts for peak efficiency relies on manipulating the excited-state pathways and energies. The methods of evaluating the efficiency of photocatalysts rely on a variety of photochemical measurements.

Herein describes contributions in photochemistry for the evaluation, development, and applications of photocatalysts with a focus on triplet photosensitizers. The development of two new methods are described for chemical actinometry and the determination of $\phi_{\Delta}$. Additionally, a model based on computational and experimental data for the prediction of the $\phi_{\Delta}$ of fluorescein based triplet photosensitizers is described. 
Finally, new applications for the use of triplet photosensitizers for the transformation of thiols to disulfides and conversion of light energy to mechanical energy were developed.

\subsection{References}

1. World Energy Council. Energy Resources: Solar. World Energy Counc. 2013 World Energy Resour. Sol. 1-28 (2013) doi:https://www.worldenergy.org/wpcontent/uploads/2013/10/WER_2013_8_Solar_revised.pdf.

2. IPCC. Summary for Policymakers. In: Global warming of $1.5^{\circ}$ C. An IPCC Special Report on the impacts of global warming. (2018).

3. S.R. Bull. Renewable energy today and tomorrow. Proc. IEEE 89, 89(8), 12161226 (2001).

4. DeLemos, J. L., Bostick, B. C., Renshaw, C. E., Stürup, S. \& Feng, X. Landfillstimulated iron reduction and arsenic release at the Coakley Superfund Site (NH). Environ. Sci. Technol. 40, 67-73 (2006).

5. Karagas, M. R. et al. Assessment of cancer risk and environmental levels of arsenic in New Hampshire. Int. J. Hyg. Environ. Health 25, 85-94 (2002).

6. Martinez, A., Norström, K., Wang, K. \& Hornbuckle, K. C. Polychlorinated biphenyls in the surficial sediment of Indiana Harbor and Ship Canal, Lake Michigan. Environ. Int. 36, 849-854 (2010).

7. Kiel, K. A. Superfund Program. Encylopedia Energy, Nat. Resour. Environ. Econ. 3, 222-225 (2013).

8. Loeb, S. K. et al. The Technology Horizon for Photocatalytic Water Treatment: Sunrise or Sunset? Environ. Sci. Technol. 53, 2937-2947 (2019).

9. Ravelli, D., Dondi, D., Fagnoni, M. \& Albini, A. Photocatalysis. A multi-faceted concept for green chemistry. Chem. Soc. Rev. 38, 1999-2011 (2009).

10. Becker, R. Theory and Interpretation of Fluorescence and Phosphorescence. (Wiley Interscience, 1969).

11. Fleming, G. R., Knight, A. W. E., Morris, J. M., Morrison, R. J. S. \& Robinson, G. W. Picosecond Fluorescence Studies of Xanthene Dyes. J. Am. Chem. Soc. 99, 4306-4311 (1977).

12. Pensack, R. D. et al. Evidence for the rapid conversion of primary photoexcitations to triplet states in seleno- and telluro- analogues of poly(3-hexylthiophene). $J$. 
Phys. Chem. B 118, 2589-2597 (2014).

13. Detty, M. R. \& Merkel, P. B. Chalcogenapyrylium dyes as potential photochemotherapeutic agents. Solution studies of heavy atom effects on triplet yields, quantum efficiencies of singlet oxygen generation, rates of reaction with singlet oxygen, and emission quantum yields. J. Am. Chem. Soc. 112, 3845-3855 (1990).

14. Farrell, K. M., Brister, M. M., Pittelkow, M., Sølling, T. I. \& Crespo-Hernández, C. E. Heavy-Atom-Substituted Nucleobases in Photodynamic Applications: Substitution of Sulfur with Selenium in 6-Thioguanine Induces a Remarkable Increase in the Rate of Triplet Decay in 6-Selenoguanine. J. Am. Chem. Soc. 140, 11214-11218 (2018).

15. Gibson, G. L., McCormick, T. M. \& Seferos, D. S. Effect of group-14 and group16 substitution on the photophysics of structurally related donor-acceptor polymers. J. Phys. Chem. C 117, 16606-16615 (2013).

16. DeRosa, M. C. \& Crutchley, R. J. Photosensitized singlet oxygen and its applications. Coord. Chem. Rev. 233-234, 351-371 (2002).

17. Zhao, J., Wu, W., Sun, J. \& Guo, S. Triplet photosensitizers: from molecular design to applications. Chem. Soc. Rev. 42, 5323-5351 (2013).

18. Hatchard, C. G. \& Parker, C. A. A New Sensitive Chemical Actinometer. II. Potassium Ferrioxalate as a Standard Chemical Actinometer. Proc. R. Soc. A Math. Phys. Eng. Sci. (1956) doi:10.1098/rspa.1956.0102.

19. Lin, T.-P. \& Gabbaï, F. P. Two-electron redox chemistry at the dinuclear core of a TePt platform: chlorine photoreductive elimination and isolation of a $\mathrm{Te}(\mathrm{V}) \mathrm{Pt}(\mathrm{I})$ complex. J. Am. Chem. Soc. 134, 12230-8 (2012).

20. Carrera, E. I., McCormick, T. M., Kapp, M. J., Lough, A. J. \& Seferos, D. S. Thermal and photoreductive elimination from the tellurium center of ??-conjugated tellurophenes. Inorg. Chem. 52, 13779-13790 (2013).

21. Han, Z. \& Eisenberg, R. Fuel from water: the photochemical generation of hydrogen from water. Acc. Chem. Res. 47, 2537-44 (2014).

22. Fukuzumi, S. Artificial photosynthesis for production of hydrogen peroxide and its fuel cells. Biochim. Biophys. Acta (2015) doi:10.1016/j.bbabio.2015.08.012.

23. Carrera, E. I. \& Seferos, D. S. Efficient halogen photoelimination from dibromo, dichloro and difluoro tellurophenes. Dalton Trans. 44, 2092-6 (2015).

24. Carrera, E. I., Lanterna, A. E., Lough, A. J., Scaiano, J. C. \& Seferos, D. S. A Mechanistic Study of Halogen Addition and Photoelimination from $\pi$-Conjugated 
Tellurophenes. J. Am. Chem. Soc. 138, 2678-2689 (2016).

25. Shingo, O., Tomoya, I., Yuichirou, H., Shunichi, F. \& Kojima, T. Photocatalytic Oxidation of Organic Compounds in Water by Using Ruthenium(II)-Pyridylamine Complexes as Catalysts with High Efficiency and Selectivity. Chem. A Eur. J. 19, 1563-1567 (2013).

26. Fujishima, A. \& Zhang, X. Titanium dioxide photocatalysis: present situation and future approaches. Comptes Rendus Chim. 9, 750-760 (2006).

27. Lang, X., Ma, W., Chen, C., Ji, H. \& Zhao, J. Selective aerobic oxidation mediated by TiO2 photocatalysis. Acc. Chem. Res. 47, 355-363 (2014).

28. Hashimoto, K., Irie, H. \& Fujishima, A. TiO2 photocatalysis: A historical overview and future prospects. Japanese J. Appl. Physics, Part 1 Regul. Pap. Short Notes Rev. Pap. 44, 8269-8285 (2005).

29. Zhao, J. \& Yang, X. Photocatalytic oxidation for indoor air purification: A literature review. Build. Environ. 38, 645-654 (2003).

30. Frimer, A. Singlet Oxygen; Physical-Chemical Aspects. (1985).

31. Frimer, A. A. The Reaction of Singlet Oxygen with Olefins: The Question of Mechanism. Chem. Rev. 79, 359-387 (1979).

32. Filatov, M. A. \& Senge, M. O. Molecular devices based on reversible singlet oxygen binding in optical and photomedical applications. Mol. Syst. Des. Eng. 1, 258-272 (2016).

33. Martinez, G. R. et al. Synthesis of a hydrophilic and non-ionic anthracene derivative, the $\mathrm{N} \mathrm{N}^{\prime}$-di-(2,3-dihydroxypropyl)-9,10-anthracenedipropanamide as a chemical trap for singlet molecular oxygen detection in biological systems. Tetrahedron 62, 10762-10770 (2006).

34. Kotani, H., Ohkubo, K. \& Fukuzumi, S. Photocatalytic oxygenation of anthracenes and olefins with dioxygen via selective radical coupling using 9-mesityl-10methylacridinium ion as an effective electron-transfer photocatalyst. J. Am. Chem. Soc. 126, 15999-16006 (2004).

35. Pedersen, S. K. et al. Aarhus sensor green: A fluorescent probe for singlet oxygen. J. Org. Chem. 79, 3079-3087 (2014).

36. Fudickar, W. \& Linker, T. Synthesis of Pyridylanthracenes and Their Reversible Reaction with Singlet Oxygen to Endoperoxides. J. Org. Chem. 82, 9258-9262 (2017).

37. Nardello, V., Brault, D., Chavalle, P. \& Aubry, J. M. Measurement of 
photogenerated singlet oxygen $(1 \mathrm{O} 2(1 \Delta(\mathrm{g})))$ in aqueous solution by specific chemical trapping with sodium 1,3-cyclohexadiene-1,4-diethanoate. J. Photochem. Photobiol. B Biol. 39, 146-155 (1997).

38. Bauch, M., Krtitschka, A. \& Linker, T. Photooxygenation of oxygen-substituted naphthalenes. J. Phys. Org. Chem. 30, 1-11 (2017).

39. Ghogare, A. A. \& Greer, A. Using Singlet Oxygen to Synthesize Natural Products and Drugs. (2016) doi:10.1021/acs.chemrev.5b00726.

40. Leonard, K. A. et al. Synthesis and evaluation of chalcogenopyrylium dyes as potential sensitizers for the photodynamic therapy of cancer. J. Med. Chem. 42, 3953-3964 (1999).

41. Kryman, M. W. et al. Organotellurium fluorescence probes for redox reactions: 9aryl-3,6-diaminotelluroxanthylium dyes and their telluroxides. Organometallics 32, 4321-4333 (2013).

42. Oba, M. et al. Formation of diaryl telluroxides and tellurones by photosensitized oxygenation of diaryl tellurides. Inorg. Chem. 49, 10680-10686 (2010).

43. You, Y., Ahsan, K. \& Detty, M. R. Mechanistic studies of the tellurium(II)/tellurium(IV) redox cycle in thiol peroxidase-like reactions of diorganotellurides in methanol. J. Am. Chem. Soc. 125, 4918-4927 (2003).

44. Oba, M., Tanaka, K., Nishiyama, K. \& Ando, W. Aerobic oxidation of thiols to disulfides catalyzed by diaryl tellurides under photosensitized conditions. J. Org. Chem. 76, 4173-4177 (2011).

45. Meerholz, A., Derek, H. R. \& Ley, S. Bis(methoxyphenyl) Telluroxide : a New , Mild Oxidising Agent. J. Chem. Commun. 755-756 (1979).

46. Detty, M. R. \& Gibson, S. L. Tellurapyrylium dyes as catalysts for oxidations with hydrogen peroxide and as scavengers of singlet oxygen. Dihydroxytelluranes as mild oxidizing agents. Organometallics 11, 2147-2156 (1992).

47. Malkin, J. Photophysical and Photochemical Properties of Aromatic Compounds. (1992).

48. Koide, Y. et al. A reversible near-infrared fluorescence probe for reactive oxygen species based on Te-rhodamine. Chem. Commun. 48, 3091 (2012).

49. Shah, S. S., Karthik, S. \& Singh, N. D. P. Vis/NIR light driven mild and clean synthesis of disulfides in the presence of $\mathrm{Cu}_{2}(\mathrm{OH}) \mathrm{PO}_{4}$ under aerobic conditions. RSC Adv. 5, 45416-45419 (2015).

50. Rubin, M. B. \& Braslavsky, S. E. Quantum yield: The term and the symbol. A 
historical search. Photochem. Photobiol. Sci. 9, 670-674 (2010).

51. Amand, B. \& Bensasson, R. Determination of triplet quantum yields by Laser Flash Absorption Spectroscopy. Chem. Phys. Lett. 34, (1975).

52. Hasebe, N. et al. Absolute phosphorescence quantum yields of singlet molecular oxygen in solution determined using an integrating sphere instrument. Anal. Chem. 87, 2360-2366 (2015).

53. Megerle, U., Lechner, R., König, B. \& Riedle, E. Laboratory apparatus for the accurate, facile and rapid determination of visible light photoreaction quantum yields. Photochem. Photobiol. Sci. 9, 1400-1406 (2010).

54. Rabek, J. F. Experimental Methods in Photochemistry and Photophysics. (John Wiley \& Sons, 1982).

55. Housecroft, C. E. \& Sharpe, A. G. Inorganic Chemistry. (Pearson Prentice-Hall, 2005).

56. Kryman, M. W. et al. Synthesis and properties of heavy chalcogen analogues of the texas reds and related rhodamines. Organometallics 33, 2628-2640 (2014).

57. Detty, M. R. Reaction Pathways of Telluroxide Equivalents. Reductive Elimination of Hydrogen Peroxide from Dihydroxytelluranes and Oxidation of Carbon via Intramolecular Transfer of Oxygen. Organometallics 10, 702-712 (1991). 


\section{Quantifying Singlet Oxygen Quantum Yields by Oxygen Consumption}

This work has been published as: Lutkus, L. V.; Rickenbach, S. S.; McCormick, T. M. Singlet Oxygen Quantum Yields Determined by Oxygen Consumption. J. Photochem. Photobiol. A Chem. 2019, 378, 131-135.

\subsection{Background}

Chromophores with significant triplet yields have found utility in fields such as photocatalytic water splitting, organic redox reactions, as well as in organic electronic applications. ${ }^{1-5}$ Triplet-photosensitizers are chromophores that show a significant rate of intersystem crossing from a singlet-excited-state to produce a triplet-excited-state and can display phosphorescence. As compared to singlet-excited-states, triplet-excited-states typically are longer lived and can undergo energy transfers to other triplet-states such as ground state oxygen $\left({ }^{3} \mathrm{O}_{2}\right)$. Energy transfer from triplet-excited-states to ${ }^{3} \mathrm{O}_{2}$ produce singlet oxygen $\left({ }^{1} \mathrm{O}_{2}\right) .{ }^{1} \mathrm{O}_{2}$ is $94 \mathrm{~kJ} / \mathrm{mol}$ higher in energy than ${ }^{3} \mathrm{O}_{2}$ and has found utility in organic synthesis due to its increased reactivity. ${ }^{6-8}{ }^{1} \mathrm{O}_{2}$ has been used in the oxidation of arenes, alkenes, and heteroatoms such as sulfur, nitrogen and phosphorous. ${ }^{9}{ }^{10}$ The production of ${ }^{1} \mathrm{O}_{2}$ can be quantified and used as a lower bound approximation for the triplet yield of a chromophore. Therefore, the measurement of ${ }^{1} \mathrm{O}_{2}$ quantum yields is crucial for the evaluation and optimization of systems employing triplet photosensitizers.

${ }^{1} \mathrm{O}_{2}$ quantum yields are often used to approximate the triplet yield of a chromophore since directly measuring the triplet yield can be challenging. If the phosphorescence quantum yield $\left(\Phi_{\mathrm{p}}\right)$ approaches unity the triplet yield can be approximated by measuring the emission quantum yield. However, most chromophores 
have competing radiative and non-radiative decay pathways making this method invalid. Advanced spectroscopic methods are required to directly quantify triplet yields with nonradiative decay pathways. ${ }^{11,12}$ In contrast, a wide variety of methods have been developed to determine the ${ }^{1} \mathrm{O}_{2}$ yield as an approximation of the triplet yield of a chromophore. ${ }^{1} \mathrm{O}_{2}$ formation is typically monitored directly by its phosphorescence around $1270 \mathrm{~nm} .{ }^{13}$ The phosphorescence can be quantified using a relative method, by comparing the emission integration of a known standard, or an absolute method using an integration sphere. Both methods rely on the excitation of the triplet photosensitizer and monitoring the phosphorescence of ${ }^{1} \mathrm{O}_{2}$. The phosphorescence of ${ }^{1} \mathrm{O}_{2}$ is typically very weak, with $\phi_{p}$ ranging from $10^{-6}$ to $10^{-2}$ in common laboratory solvents, thus a very sensitive IR detector is required. ${ }^{13,14}$ To avoid the need for an IR detector, reactions with an easily monitored spectroscopic signature have found utility as ${ }^{1} \mathrm{O}_{2}$ sensors.

A wide variety of chemical traps for ${ }^{1} \mathrm{O}_{2}$ have been used. ${ }^{7}, 15-17$ Among the most common are 9,10-disubstitued anthracene compounds and 1,3-diphenylisobenzofuran which react with ${ }^{1} \mathrm{O}_{2}$ to form endoperoxides and an diketone, respectively. ${ }^{18,19}$ For both, the progress of the reaction is monitored by the change in absorbance of the compound by UV-vis absorption spectroscopy. Synthetic strategies have been used to provide different substituents at the 9 and 10 positions to increase solubility of anthracene traps in polar solvents. ${ }^{16,20}$ Challenges can arise if the absorbance range of the photosensitizer in question overlaps with the chemical trap making these measurements difficult. ${ }^{21} \mathrm{To}$ overcome absorbance overlaps, the change in fluorescence from the chemical trap may also be monitored..$^{21,22}$ 
More specialized chemical traps have been developed to detect ${ }^{1} \mathrm{O}_{2}$ by visible fluorescence. These traps typically contain a fluorescein core structure with a 9,10disubstitued anthracene substituent to trap the ${ }^{1} \mathrm{O}_{2}$ such as Aarhus Sensor Green (ASG) and the commercially available Singlet Oxygen Sensor Green (SOSG) (Scheme 2.1). ${ }^{22,23}$ SOSG has been shown to have great selectivity towards ${ }^{1} \mathrm{O}_{2}$ compared to other reactive oxygen species. However, complications arise since the endoperoxide of SOSG can itself create ${ }^{1} \mathrm{O}_{2}$ with a $\phi_{\Delta}$ up to 0.20 when excited. ${ }^{23}$ ASG and its endoperoxide have been shown to have a much lower $\phi_{\Delta}$, however, (to our knowledge) this sensor is not commercially available.

As describe above, most ${ }^{1} \mathrm{O}_{2}$ measurements monitor the concentration of the chemical trap rather than the oxygen itself. Herein we propose that the amount of oxygen trapped from such reactions can be measured directly, which eliminates the need for specialty ${ }^{1} \mathrm{O}_{2}$ sensors with unique spectroscopic signatures. Irradiation of triplet chromophores in the presence of a chemical trap has been shown to result in the consumption of oxygen over time. ${ }^{7}$ Production or consumption of gases can be monitored by the accompanying pressure change in a sealed system. Pressure sensors (transducers) are becoming common in many photochemistry labs to monitor photo-catalytic reactions such as water splitting to produce $\mathrm{H}_{2}$ gas. ${ }^{24,25}$ Gas producing reactions, such as water splitting, produce a pressure increase, while gas consuming reactions such as those with ${ }^{1} \mathrm{O}_{2}$ can be monitored by a pressure decrease. 
Herein we describe a new method for determining the ${ }^{1} \mathrm{O}_{2}$ quantum yield using common laboratory chemicals that does not rely on spectroscopic measurements. We use the reaction of ${ }^{1} \mathrm{O}_{2}$ with dimethyl sulfoxide (DMSO). ${ }^{1} \mathrm{O}_{2}$ reacts with DMSO to afford dimethyl sulfone, resulting in the consumption of atmospheric oxygen over time that can be monitored by a corresponding pressure decrease within a closed system. The rate of pressure decrease was found to be directly related to the ${ }^{1} \mathrm{O}_{2}$ quantum yield of the chromophore in question.

Scheme 2.1. Structures of selected ${ }^{1} \mathrm{O}_{2}$ traps

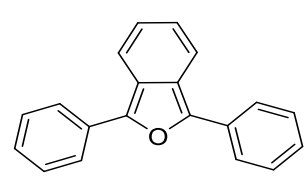

DPBF

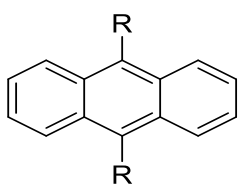

9,10-disubstitued anthracene

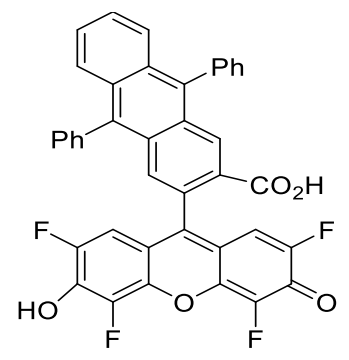

ASG<smiles>Cc1c2ccccc2c(C2=Cc3c(cc(=O)c4ccccc4c3-c3ccc(C(=O)O)cc3C(=O)O)Oc3cc(O)c(Cl)cc32)c2ccccc12</smiles>

SOSG

\subsection{Results and Discussion}

Measuring ${ }^{1} \mathrm{O}_{2}$ quantum yields typically relies on monitoring the reaction progress of singlet oxygen with a chemical trap, which is observed by a change in fluorescence or absorbance. Monitoring the consumption of oxygen, rather than the oxidation of these 
chemical traps, allows for the use of compounds that react with singlet oxygen but do not produce easily observable spectroscopic changes. DMSO was chosen as the singlet oxygen trap because it is commonly available in most research laboratories. Furthermore, DMSO is a polar aprotic solvent which can dissolve a wide range of chromophores. DMSO reacts with singlet oxygen in a 2:1 to stoichiometry to afford dimethyl sulfone

Scheme 2.2. Reaction of DMSO with singlet oxygen

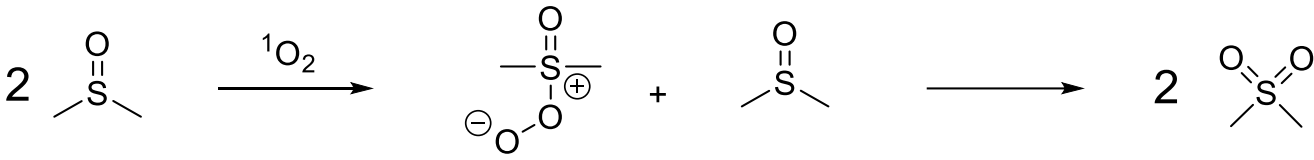

(Scheme 2.2). Consequently, oxygen gas from the headspace is dissolved, to restore equilibrium, resulting in a decrease in pressure over time.

\subsubsection{Oxygen consumption from Rose Bengal in DMSO}

As a proof of concept, Rose Bengal, a well-known singlet oxygen sensitizer, was used as a reference to compare to other dyes. Solutions containing Rose Bengal $\left(1 \times 10^{-5}\right.$ $\mathrm{M}, 10 \mathrm{~mL}$ ) in DMSO were sealed in $50 \mathrm{~mL}$ reaction vessels containing $40 \mathrm{~mL}$ of atmospheric headspace. The reaction vessels were sealed with caps fitted with gage pressure sensors, that were read into a LabView ${ }^{\mathrm{TM}}$ program. The solutions were irradiated with red, green, and/or blue LEDs (to best match the absorption spectra of the photosensitizers) for five hours. The pressure change was recorded every two minutes for each sample resulting in 300 data points. The samples were consistently stirred at 500 rpm to assist in dissolving oxygen from the headspace. 
A control sample containing only DMSO was monitored to account for the pressure change caused by temperature increase from irradiation. The pressure change at each time point of the control sample was then subtracted from the samples containing dye. After averaging the triplicate data, a linear fit was applied to determine the rate of the pressure decrease. The linear fit from irradiating solutions of Rose Bengal in triplicate showed a consistent pressure decrease plot with an average slope of $-2.248( \pm 0.007) \times$ $10^{-5} \mathrm{psi} \cdot \mathrm{s}^{-1}$. Applying a linear fit to the three individual samples resulted in linear fits with slopes within $7.5 \%$ of the average slope indicating good precision from this method.

The resulting pressure decrease over time shows a pseudo-zeroth order rate of oxygen consumption. The zeroth order plots are expected since both DMSO and the dissolved oxygen are in excess for the duration of the measurement and the concentration remains essentially unchanged. The rate of the pressure decrease is expected to be constant as long as there is no degradation to the photosensitizer and enough oxygen is dissolved to maintain the reaction, resulting in a linear decrease in pressure.

The gas composition of the headspace of the reaction vessels was analyzed by a Quantitative Gas Analyzer (QGA), before and after irradiation, to verify that only oxygen had been consumed. The concentration of the oxygen in the headspace after irradiation was nearly $2.7 \%$ less for each sample containing Rose Bengal than before. The average rate of decrease from the irradiated Rose Bengal samples was used as a relative standard for other photosensitizers, using the accepted singlet oxygen quantum yield of 0.76 as a reference as discussed below. ${ }^{26}$ 


\subsubsection{Calculation of singlet oxygen yields}

The rate of oxygen consumption by Rose Bengal was compared to five other known photosensitizers to correlate the rate of pressure decrease from oxygen consumption to the singlet oxygen quantum yield. However, to accurately determine the quantum yields of the other dyes the absorption spectrum of the dye and the spectrum of the light source was considered through relative absorbance, $I$ (equation 1 ). ${ }^{27}$ Using $I$ in the calculation of the singlet oxygen yields allows for the translation of results from the rate of pressure decrease from chromophores with different absorbance profiles irradiated using non-monochromatic light.

Equation 2.1. Calculation of relative absorbance

$$
I=\int_{400 \mathrm{~nm}}^{900 \mathrm{~nm}} i(\lambda)\left(1-10^{-A b s(\lambda)}\right) d \lambda
$$

The integral of $i$, the intensity of the light source at a given wavelength $(\lambda)$, with respect to the absorbance of the dye $(A b s)$ at the given wavelength was used to produce the absorbance intensity $(I)$. The area was integrated from $400 \mathrm{~nm}$ to $900 \mathrm{~nm}$ in order to incorporate the full spectral range of the LEDs and encompass the absorbance range of the dyes. A visual representation of the calculation is shown below. 

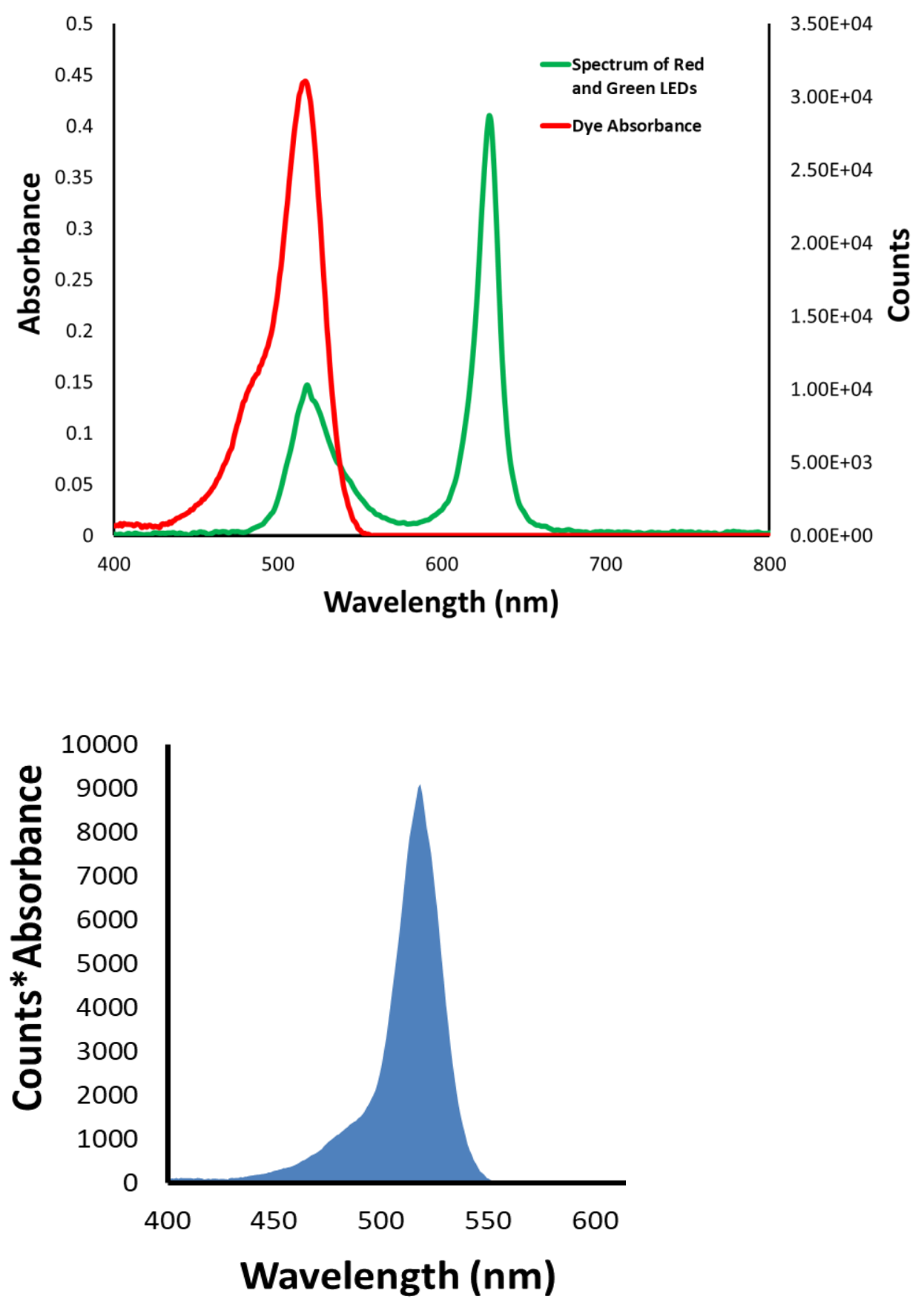

Figure 2.1. Absorbance spectrum of Rose Bengal and spectrum of green and red LED intensity (top). Absorbance at a given wavelength times the light intensity at that wavelength (bottom). 
Equation 2.2. Calculation of ${ }^{1} \mathrm{O}_{2}$ quantum yield from oxygen consumption

$$
\phi_{\Delta \text { unknown }}=\frac{I_{\text {Rose Bengal }}}{I_{\text {unknown }}} \times \frac{k_{\text {unknown }}}{k_{\text {Rose Bengal }}} \times \phi_{\Delta \text { Rose Bengal }}
$$

The singlet oxygen quantum yield was calculated comparing the rate of pressure decrease $(k)$ and relative absorbance of the dye relative to Rose Bengal (equation 2.1). ${ }^{27}$ Utilizing the rate of pressure decrease rather than the total pressure decrease allows for results to be compared over different irradiation periods.
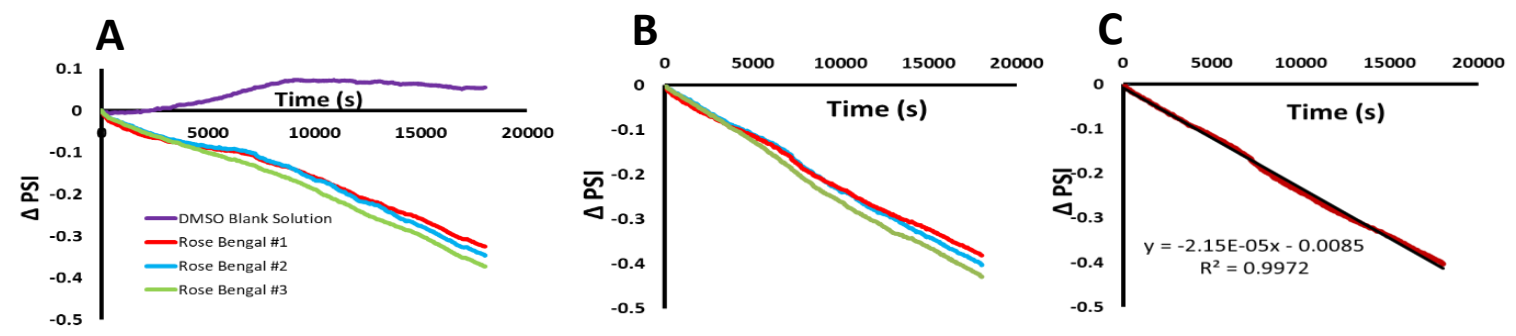

Figure 2.2. A Uncorrected pressure change over time from irradiation of DMSO and triplicate solutions of DMSO containing Rose Bengal. B Adjusted pressure change of triplicate samples of Rose Bengal after subtracting the pressure change of the DMSO blank solution. C Average rate of pressure decrease over time of the triplicate samples of Rose Bengal (orange) and the linear fit (black).

\subsubsection{Experimental singlet oxygen quantum yields}

The resulting calculated singlet oxygen quantum yields are summarized in Table 2.1 for fluorescein, Eosin Y, Eosin B, methylene blue and tris(bypridine)-ruthenium(II) $\left(\mathrm{Ru}(\mathrm{bpy})_{3}\right)$ using Rose Bengal as a reference. The calculated singlet oxygen quantum yields are in good agreement with previously reported values for the photosensitizers in water or ethanol. All dyes produced pseudo-zeroth order plots of pressure decrease. The rate of the pressure decrease was determined by applying a linear fit across the average of 
triplicate samples. A sample of only DMSO was run simultaneously and the pressure change of this blank was subtracted from each photosensitizer sample before being averaged. All $R^{2}$ values were above 0.95 , with the exception of fluorescein $\left(R^{2}=0.88\right)$, indicating that using the slope of the linear fit was a good estimate for the rate of oxygen

Table 2.1. Calculated singlet oxygen quantum yields from rates of pressure decrease

\begin{tabular}{cccc}
\hline Photosensitizer & Calculated $\boldsymbol{\phi}_{\Delta}$ & $\begin{array}{c}\text { Literature } \boldsymbol{\phi}_{\Delta} \text { in } \\
\mathbf{H}_{2} \mathbf{O}\end{array}$ & $\begin{array}{c}\text { Literature } \boldsymbol{\phi}_{\Delta} \text { in } \\
\text { EtOH }\end{array}$ \\
\hline Fluorescein & $0.066 \pm 0.001$ & $0.03,0.06$ & $0.13,0.03$ \\
Eosin Y & $0.611 \pm 0.003$ & $0.52,0.57$ & $0.60,0.42$ \\
Methylene Blue & $0.491 \pm 0.003$ & $0.60,0.52$ & $0.52,0.50$ \\
Eosin B & $0.370 \pm 0.003$ & 0.52 & 0.38 \\
Ru(bpy) ${ }_{3}$ & $0.656 \pm 0.008$ & $0.22 *$ & $0.73 * *$ \\
Rose Bengal & $\mathrm{N} / \mathrm{A}$ & Reference Value 0.76 \\
\hline
\end{tabular}

* Reported value in $\mathrm{D}_{2} \mathrm{O}$ ** Reported value in $\mathrm{CD}_{3} \mathrm{OD}^{7}$

consumption.

The plots of the pressure decrease over time for the photosensitizers are shown in Figure 2.3. The sensitivity of the method was demonstrated by using fluorescein, which has reported singlet oxygen quantum yield an order of magnitude less than Rose Bengal. The increase of temperature after irradiation with the LEDs did cause an initial increase in pressure, even after subtraction of the pressure change from the blank solution, as observed in the pressure for fluorescein and methylene blue (Figure 2.3). However, the five hour irradiation time was sufficient to see an overall decrease in pressure due to 
oxygen consumption even with the low singlet oxygen quantum yield of fluorescein.

Other fluorescein derivatives, Eosin $\mathrm{Y}$ and Eosin B, showed a more dramatic pressure decrease compared to fluorescein in line with their higher triplet yields. The importance of incorporating the relative absorbance, $I$, was demonstrated by comparing Eosin $\mathrm{Y}$ and Eosin B. The slope of pressure decrease of Eosin B was half that of Eosin Y despite similar triplet quantum yields. However, after incorporating the respective relative absorbance for both Eosin $\mathrm{Y}$ and Eosin $\mathrm{B}$ their singlet oxygen quantum yields were in good agreement with literature values. Methylene blue also showed a singlet oxygen

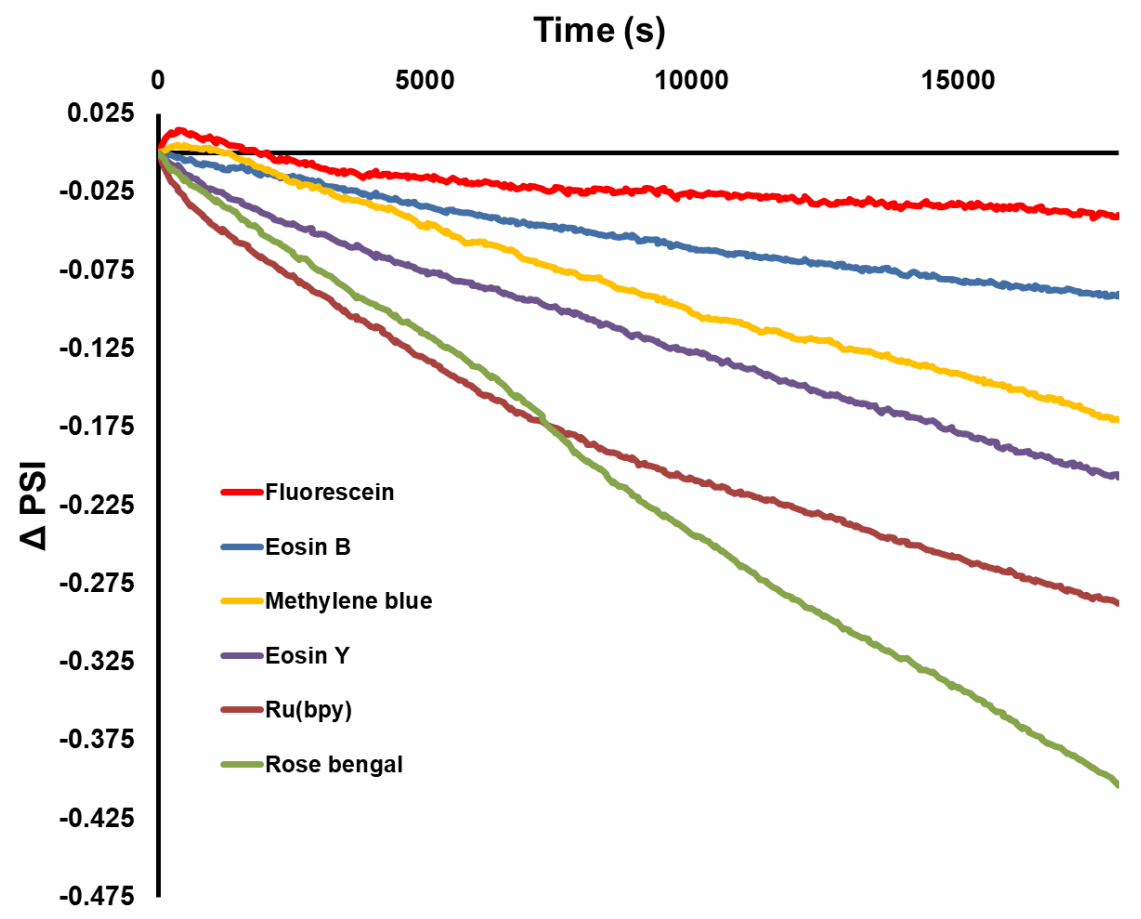

Figure 2.3. The average pressure change over time for triplicate runs of 6 different photosensitizers irradiated by red, green, or blue LEDs in DMSO. The slope of the pressure change was monitored every 2 minutes for 5 hours. 
quantum yield consistent from data previously reported in water. To accommodate the absorbance range of $\mathrm{Ru}(\mathrm{bpy})_{3}$, blue and green LEDs were used rather than the green and/or red LEDs, which were used to irradiate all the other photosensitizers. Ru(bpy) ${ }_{3}$ has been reported to have greatly varying singlet oxygen yields in different solvents. In methanol, the reported singlet oxygen yield for $\mathrm{Ru}(\text { byp })_{3}$ is 0.70 , however, in water this yield diminishes to 0.22 . We found the singlet oxygen yield for Ru(byp) 3 in DMSO was closer to that of methanol at 0.66 .

\subsubsection{Oxygen consumption in mixed solvent systems}

The solvent of choice can have a great influence on the singlet oxygen quantum yield. The minimum amount of DMSO needed to observe a statistically significant

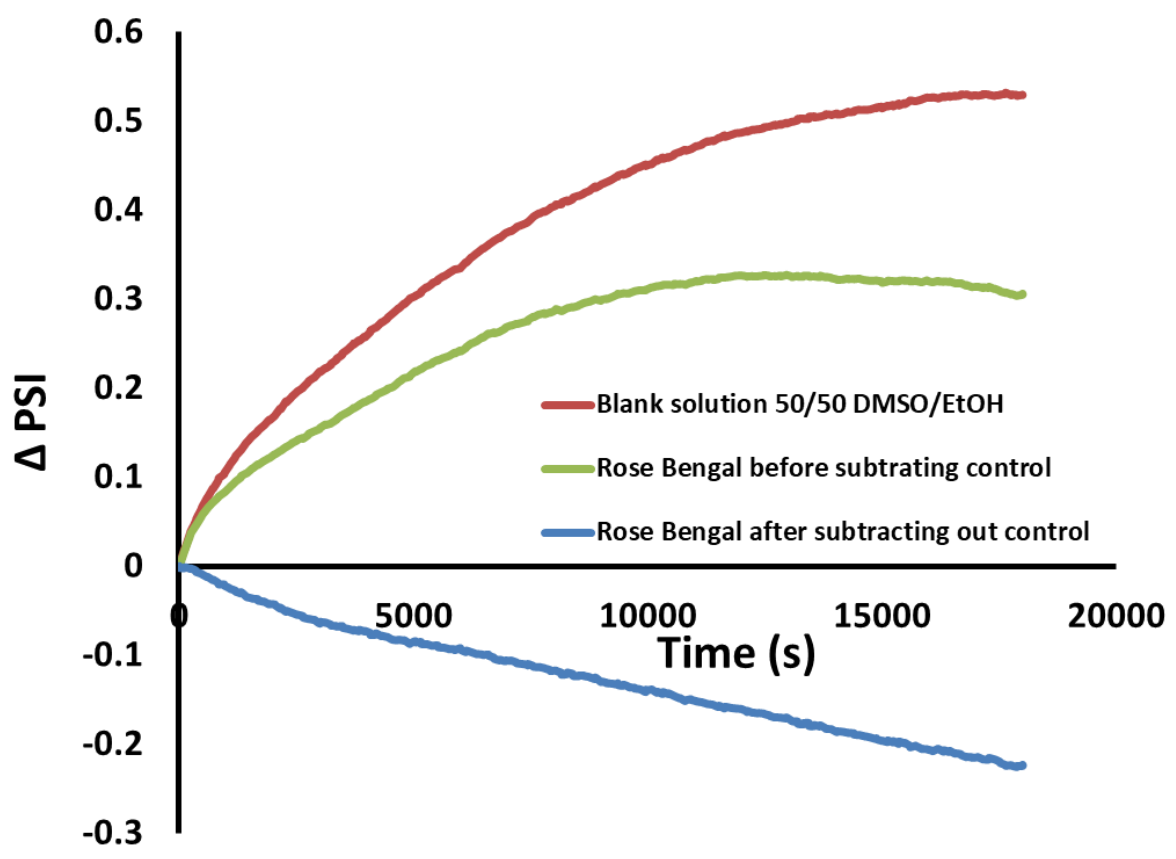

Figure 2.4. Pressure change over time of irradiated solutions of Rose Bengal in 50/50 $\mathrm{DMSO} / \mathrm{EtOH}$ resulting in an increase in pressure (green line) before adjusting for vapor pressure by subtracting the blank solution (blue line). 
pressure decreased was examined by mixing DMSO with ethanol. The addition of ethanol proved to be troublesome as the increase in vapor pressure made measurements difficult below $50 \%$ DMSO by volume. At 50\% DMSO a solution containing Rose Bengal resulted in a net increase in pressure, however, after subtracting the respective blank solution containing 50\% DMSO and 50\% ethanol a pressure decrease with a slope of $-1.116( \pm 0.006) \times 10^{-5} \mathrm{psi} \cdot \mathrm{s}^{-1}$ was observed Figure 2.4. Solutions with $25 \%$ DMSO and $75 \%$ ethanol were attempted, however, fluctuations of the pressure resulted in inconsistent plots even after subtracting the control sample but careful temperature control could overcome this limitation.

\subsubsection{Reaction of singlet oxygen with triphenylphosphine}

To investigate the utility of this method with other substrates beyond DMSO the pressure change from the reaction of singlet oxygen with triphenylphosphine was also examined. The reaction of triphenylphosphine with singlet oxygen produces triphenylphosphine oxide in a similar 2:1 ratio as DMSO with singlet oxygen. Use of triphenylphosphine allows for dissolution in nonpolar solvents that DMSO may not be miscible in.

1-Butanol was chosen as the solvent to accommodate the solubility of both Rose Bengal and triphenylphosphine and minimize vapor pressure. Irradiation of solutions of Rose Bengal and saturated triphenylphosphine $(0.08 \mathrm{M})$ resulted in a rate of pressure decrease of $-1.421( \pm 0.004) \times 10^{-5} \mathrm{psi} \cdot \mathrm{s}^{-1}($ Figure 2.5). Again, the the pressure decrease over time was linear indicating a pseudo-zeroth order reaction rate. Triphenlyphosphine is 
known to oxidize over time in normal atmospheric conditions, however, no indication of pressure change was apparent from the control sample containing only 1-butanol and triphenylphosphine over the course of the reaction.

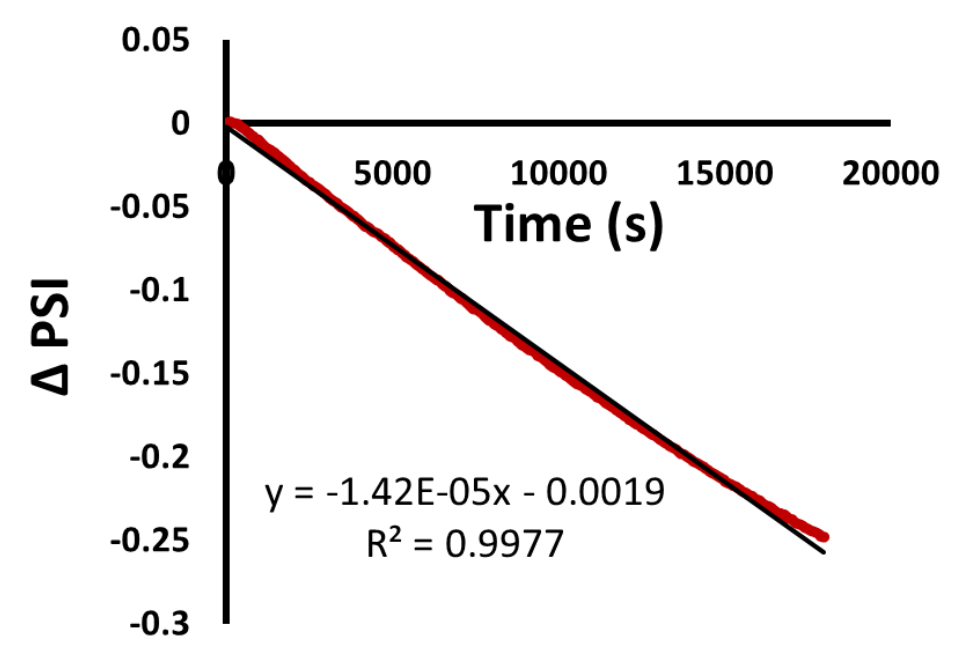

Figure 2.5. Pressure decrease over time from irradiation of Rose Bengal with triphenylphosphine in 1-butanol.

\subsubsection{Monitoring oxygen consumption by water displacement}

To eliminate the need of pressure sensors; a manual method was developed that measures water displacement from oxygen consumption that can be assembled using simple equipment available in most labs. The same pressure sensor tube with Rose Bengal in DMSO was used for a consistent irradiation area but it was fitted with a septum instead of the pressure sensor cap. A hose with a needle puncturing the septum was connected to a $1 \mathrm{~mL}$ pipette submerged in a graduated cylinder of water. Air was injected through the septum of the reaction vessel to displace the water in the pipette such that the water level started at zero. The sample was irradiated with green LEDs and the oxygen 
consumption was monitored by the rise of the water in the pipette. The water level in the pipette was monitored every hour for five hours resulting in consumption of $\sim 0.8 \mathrm{~mL}$ of

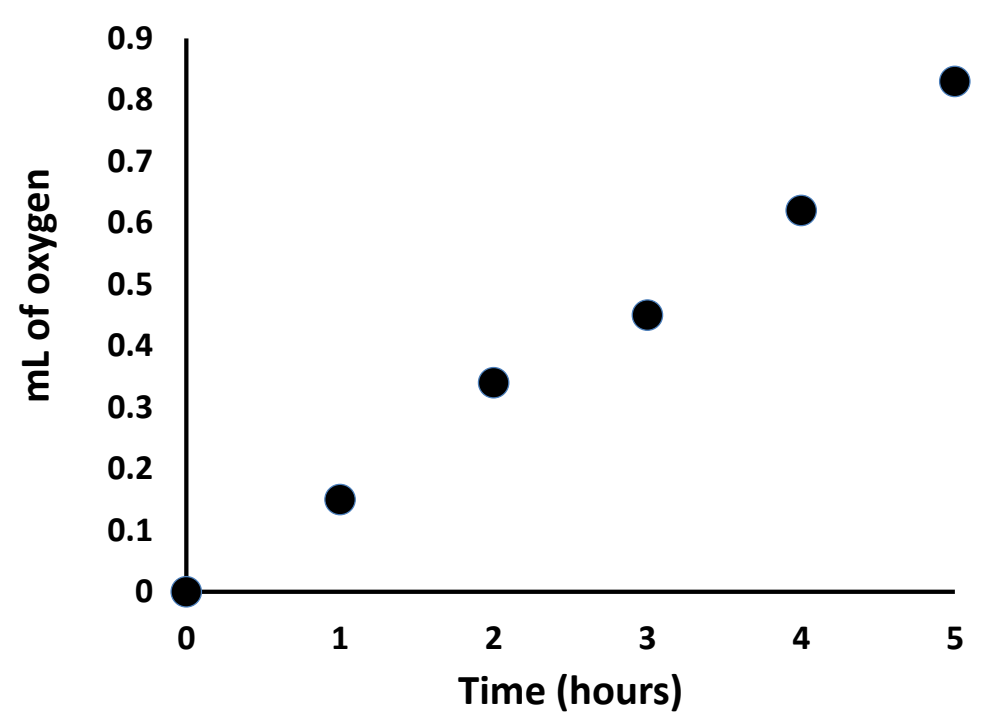

Figure 2.6. Oxygen consumption over time of an irradiated solution of Rose Bengal in DMSO measured by the water displacement method oxygen (Figure 2.6)

\subsection{Conclusion}

We have developed a method for determining the ${ }^{1} \mathrm{O}_{2}$ quantum yield of a triplet photosensitizer by monitoring the rate of pressure decrease upon irradiation of the photosensitizer in DMSO. The method can be performed in sealed systems under 
atmospheric conditions and does not require specialized chemical traps. Pseudo zeroth order plots of pressure decrease were shown for each photosensitizer. The rate of decrease in pressure was found to be related to the singlet oxygen quantum yield of the photosensitizer. The inclusion of the absorbance intensity term allows for the translation of results between dyes that absorb at different wavelengths and for different light sources. We focused on using DMSO to capture the singlet oxygen, however, the method can be adapted and used for a broad range of substrates that react with singlet oxygen. The need for pressure sensors can be substituted with a manual method by measuring the displacement of water over time. This method allows for the determination of singlet oxygen quantum yields without the need for specialty traps or spectroscopic measurements.

\subsection{Materials and Methods}

\subsubsection{Chemicals used}

Rose Bengal, Eosin Y, Eosin B, methylene blue, tris(bypyridine)ruthenium (II) hexafluorophosphate, and fluorescein were obtained by commercial sources and used without further purification. Stock solutions of the dyes $\left(10^{-5} \mathrm{M}\right)$ were created in DMSO and stored in light free conditions. The $\mathrm{pH}$ was adjusted for Rose Bengal, Eosin $\mathrm{Y}$, and fluorescein by adding $\mathrm{NaOH}(1 \mathrm{M})$ dropwise until no change in the UV-vis spectrum occurred.

\subsubsection{Oxygen consumption measurements}


Pressure sensor experiments were carried out in sealed $50 \mathrm{~mL}$ reaction vessels with Omega gage pressure sensors to monitor the pressure every two minutes for five hours using the TracerDAQ Stripchart program. Red, green, and blue LED ring lights lining a steal tube were used to evenly irradiate up to twelve samples held in a Radleys Carousel 12 cooled reaction station and stirred at 500 RPM. The entire apparatus was covered in order to avoid irradiation from room lights. Samples of each photosensitizer $(10 \mathrm{~mL})$ were run in triplicate, with a single control solution containing only DMSO. The resulting rate was calculated from the average slope of the triplicate samples after subtraction of the pressure change of the blank solution.

\subsubsection{Calculation of absorbance intensity $(I)$}

The spectrum of the red, green, and blue LEDs, used to irradiate the samples, was measured using a Stellar Net Spectra Wiz in scope mode. The absorbance spectrum of the dyes was collected on UV-3600 Shimadzu UV-Vis NIR spectrometer. The spectra were used as detailed in equation 2.1 and the integral was determined using the Trapezoid Method.

\subsubsection{Water displacement method}

A pressure sensor tube containing Rose Bengal $\left(10^{-5} \mathrm{M}\right)$ in DMSO $(10 \mathrm{~mL})$ was set up as shown in Figure 2.7. The solution was irradiated with green LEDs and the water level in the pipette was monitored every hour. 


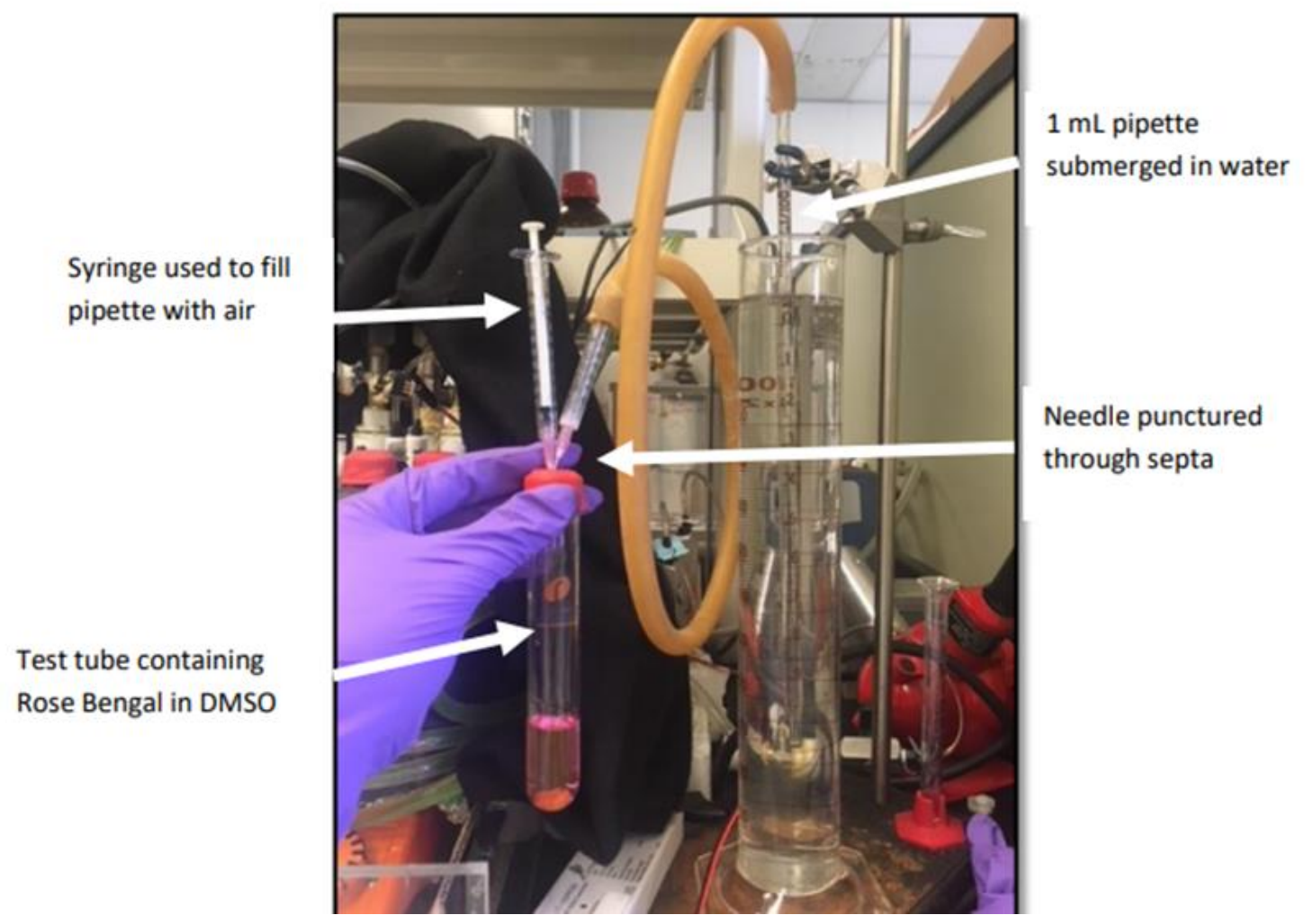

Figure 2.7. General set up for manual method to follow oxygen consumption over time.

\subsection{References}

1. Han, Z. \& Eisenberg, R. Fuel from water: the photochemical generation of hydrogen from water. Acc. Chem. Res. 47, 2537-44 (2014).

2. Kryman, M. W., Davies, K. S., Linder, M. K., Ohulchanskyy, T. Y. \& Detty, M. R. Selenorhodamine photosensitizers with the Texas-red core for photodynamic therapy of cancer cells. Bioorganic Med. Chem. 23, 4501-4507 (2015).

3. Shingo, O., Tomoya, I., Yuichirou, H., Shunichi, F. \& Kojima, T. Photocatalytic Oxidation of Organic Compounds in Water by Using Ruthenium(II)-Pyridylamine Complexes as Catalysts with High Efficiency and Selectivity. Chem. A Eur. J. 19, 1563-1567 (2013).

4. Huang, L., Zhao, J., Guo, S., Zhang, C. \& Ma, J. Bodipy derivatives as organic 
triplet photosensitizers for aerobic photoorganocatalytic oxidative coupling of amines and photooxidation of dihydroxylnaphthalenes. J. Org. Chem. 78, 56275637 (2013).

5. Luo, Y. \& Aziz, H. Correlation between triplet-triplet annihilation and electroluminescence efficiency in doped fluorescent organic light-emitting devices. Adv. Funct. Mater. 20, 1285-1293 (2010).

6. Kearns, D. R. PHYSICAL AND CHEMICAL PROPERTIES OF SINGLET MOLECULAR OXYGEN. 1970, 395-427 (1971).

7. DeRosa, M. C. \& Crutchley, R. J. Photosensitized singlet oxygen and its applications. Coord. Chem. Rev. 233-234, 351-371 (2002).

8. Hill, J. E. et al. Selenorhodamine Photosensitizers for Photodynamic Therapy of P -Glycoprotein-Expressing Cancer Cells. J. Med. Chem. 57, 8622-8634 (2014).

9. Baciocchi, E., Del Giacco, T., Lanzalunga, O. \& Lapi, A. Singlet oxygen promoted carbon-heteroatom bond cleavage in dibenzyl sulfides and tertiary dibenzylamines. Structural effects and the role of exciplexes. J. Org. Chem. 72, 9582-9589 (2007).

10. Ghogare, A. A. \& Greer, A. Using Singlet Oxygen to Synthesize Natural Products and Drugs. (2016) doi:10.1021/acs.chemrev.5b00726.

11. Amand, B. \& Bensasson, R. Determination of triplet quantum yields by Laser Flash Absorption Spectroscopy. Chem. Phys. Lett. 34, (1975).

12. Hiromitsu, I. \& Kevan, L. Correlation between Photoexcited Triplet State Yield and Photoionization Yield of Chlorophyll a in Frozen Phosphatidylcholine Vesicles Studied by Electron Spin Resonance Spectroscopy. J. Phys. Chem 32183223 (1989) doi:10.1021/j100345a066.

13. Khan, A. U. \& Kasha, M. Direct spectroscopic observation of singlet oxygen emission at $1268 \mathrm{~nm}$ excited by sensitizing dyes of biological interest in liquid solution. Proc. Natl. Acad. Sci. U. S. A. 76, 6047-6049 (1979).

14. Hasebe, N. et al. Absolute phosphorescence quantum yields of singlet molecular oxygen in solution determined using an integrating sphere instrument. Anal. Chem. 87, 2360-2366 (2015).

15. Sivéry, A., Anquez, F., Pierlot, C., Aubry, J. M. \& Courtade, E. Singlet oxygen (1O2) generation upon $1270 \mathrm{~nm}$ laser irradiation of ground state oxygen (3O2) dissolved in organic solvents: Simultaneous and independent determination of 1O2production rate and reactivity with chemical traps. Chem. Phys. Lett. 555, 252-257 (2013).

16. Botsivali, M. \& Evans, D. F. A new trap for singlet oxygen in aqueous solution. $J$. 
Chem. Soc. Chem. Commun. 24, 1114-1116 (1979).

17. Nardello, V., Brault, D., Chavalle, P. \& Aubry, J. M. Measurement of photogenerated singlet oxygen $(1 \mathrm{O} 2(1 \Delta(\mathrm{g})))$ in aqueous solution by specific chemical trapping with sodium 1,3-cyclohexadiene-1,4-diethanoate. J. Photochem. Photobiol. B Biol. 39, 146-155 (1997).

18. Kotani, H., Ohkubo, K. \& Fukuzumi, S. Photocatalytic oxygenation of anthracenes and olefins with dioxygen via selective radical coupling using 9-mesityl-10methylacridinium ion as an effective electron-transfer photocatalyst. J. Am. Chem. Soc. 126, 15999-16006 (2004).

19. Greci, L. On The Use Of 1, 3-Diphenylisobenzofuran Reactions With Carbon And Oxygen Centered Radicals In Model And Natural Systems. Res. Chem. Intermed 19, 395-396 (1993).

20. Martinez, G. R. et al. Synthesis of a hydrophilic and non-ionic anthracene derivative, the N,N'-di-(2,3-dihydroxypropyl)-9,10-anthracenedipropanamide as a chemical trap for singlet molecular oxygen detection in biological systems. Tetrahedron 62, 10762-10770 (2006).

21. Ho-Wu, R., Yau, S.-H. \& Goodson III, T. Efficient Singlet Oxygen Generation in Metal Nanoclusters for Two-Photon Photodynamic Therapy Applications. J. Phys. Chem. B 10073-10080 (2017) doi:10.1021/acs.jpcb.7b09442.

22. Marazzi, M. et al. Photophysics of the Singlet Oxygen Sensor Green Chromophore: Self-Production of 1 O 2 Explained by Molecular Modeling. J. Phys. Chem. B 7586-7592 (2017) doi:10.1021/acs.jpcb.7b04383.

23. Pedersen, S. K. et al. Aarhus sensor green: A fluorescent probe for singlet oxygen. J. Org. Chem. 79, 3079-3087 (2014).

24. Li, G., Mark, M. F., Lv, H., McCamant, D. W. \& Eisenberg, R. RhodaminePlatinum Diimine Dithiolate Complex Dyads as Efficient and Robust Photosensitizers for Light-Driven Aqueous Proton Reduction to Hydrogen. J. Am. Chem. Soc. 140, 2575-2586 (2018).

25. Kagalwala, H. N., Chirdon, D. N., Mills, I. N., Budwal, N. \& Bernhard, S. LightDriven Hydrogen Generation from Microemulsions Using Metallosurfactant Catalysts and Oxalic Acid. Inorg. Chem. 56, 10162-10171 (2017).

26. Wilkinson, F., Ross, A. \& Helman, P. Quantum Yields for the Photosensitized Formation of the Lowest Electronically Excited Singlet State of Molecular Oxygen in Solution. J. Phys. Chem. A 22, 113-256 (1992).

27. Hoebeke, M. \& Damoiseau, X. Determination of the singlet oxygen quantum yield 
of bacteriochlorin a: a comparative study in phosphate buffer and aqueous dispersion of dimiristoyl-L-alpha-phosphatidylcholine liposomes. Photochem.

Photobiol. Sci. 1, 283-287 (2002). 


\section{Photocatalytic Aerobic Oxidation Using a Tellurhodamine Chromophore}

This work has been published as: Lutkus, L. V.; Irving, H. E.; Davies, K. S.; Hill, J. E.; Lohman, J. E.; Eskew, M. W.; Detty, M. R.; McCormick, T. M. Photocatalytic Aerobic Thiol Oxidation with a Self-Sensitized Tellurorhodamine Chromophore. Organometallics 2017, acs.organomet.7b00166.

\subsection{Background}

Homogeneous metal-catalyzed aerobic oxidation is a widely used method for small molecule transformations but often is restricted by the stoichiometric use of heavy metal cooxidants. ${ }^{1}$ The use of photo-induced, redox catalysis for such transformations has increased in recent years due to its potential for green production of small molecules at both laboratory and industrial scale. ${ }^{2-4}$ Photocatalysts like $\mathrm{TiO}_{2}$ that utilize atmospheric oxygen as an oxidant are only active in the UV region, which drastically reduces their light absorption efficiency. ${ }^{5}$ Organic dyes have been developed as photo-redox catalysts for a variety of transformations including the oxidation of thiols to disulfides, ${ }^{6}$ antiMarkovnikov additions to alkenes, ${ }^{7,8}$ and oxidation of amines to iminium salts. ${ }^{9}$ Chalcogenorhodamine dyes such as those shown in Scheme 3.1 are similar in structure to the rhodamine, oxazine, thiazine, and azine dyes that have been used as visible-lightdriven photocatalysts in reactions that use singlet oxygen $\left({ }^{1} \mathrm{O}_{2}\right)$ as an oxidant. ${ }^{10}$ While both seleno- and tellurorhodamines generate ${ }^{1} \mathrm{O}_{2}$ efficiently upon irradiation, 
tellurorhodamines undergo photo-oxidation to telluroxides while selenorhodamines do not undergo oxidation to the corresponding selenoxides under similar conditions. ${ }^{11,12}$ Organotellurium compounds have been used as redox auxiliaries with a porphyrin photosensitizer for photocatalytic aerobic thiol oxidation. ${ }^{6}$ Organotellurium compounds have also been used as catalysts for the oxidation of thiols to disulfides with $\mathrm{H}_{2} \mathrm{O}_{2}{ }^{13-16}$ The proposed active oxidant in these systems is a telluroxide - a Te(IV) compound - that is reduced back to the corresponding telluride - a Te(II) compound - by thiol oxidation. Glutathione has been used as a stoichiometric reducing agent for telluroxides formed by either ${ }^{1} \mathrm{O}_{2}$ oxidation ${ }^{11,12}$ or by hypochlorite oxidation ${ }^{17}$ of tellurium-containing chromophores. Telluroxides have also been shown to oxidize a variety of substrates without the addition of cooxidants. ${ }^{18}$

As an oxidant, ${ }^{1} \mathrm{O}_{2}$ is advantageous, both economically and environmentally.

However, a short lifetime, limited scope, and lack of selectivity hinder the use of ${ }^{1} \mathrm{O}_{2}$ for many aerobic oxidation reactions. ${ }^{19}$ The ease of ${ }^{1} \mathrm{O}_{2}$ oxidation of tellurides to telluroxides, which can then be used as a chemical oxidant, can be extended to tellurium chromophores, which self-sensitize the production of ${ }^{1} \mathrm{O}_{2}$ to produce catalytic systems requiring only atmospheric $\mathrm{O}_{2}$, water and visible light irradiation.

Scheme 3.1. Oxidation of tellurorhodamine 1 to tellurorhodamine telluroxide 2 with ${ }^{1} \mathrm{O}_{2}$ and water and the structure of selenorhodamine 3 .

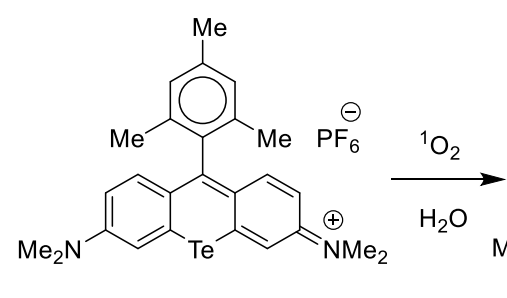

1

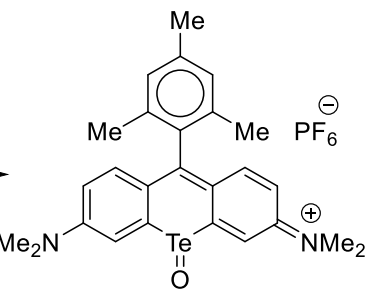

2

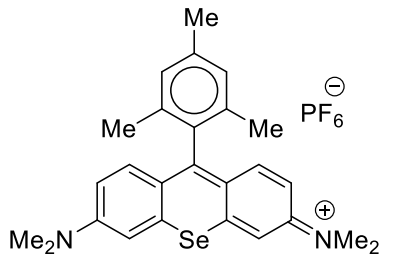

3 
Herein, we report the use of tellurorhodamine $\mathbf{1}$ (Scheme 3.1) as a visible-light-active photocatalyst for the selective aerobic oxidation of thiols to disulfides under ambient temperature and pressure in air and provide a detailed mechanism for its activity based on experimental results and DFT calculations. The role of telluroxide $\mathbf{2}$ (Scheme 3.1) as the active form of the catalyst is described. The performance of $\mathbf{1}$ is contrasted with the performance of selenorhodamine $\mathbf{3}$ as a photocatalyst, which does not form the corresponding selenoxide intermediate.

\subsection{Results and Discussion}

\subsubsection{Photophysical properties of chalcogenorhodamine dyes 1 and 3}

Tellurorhodamine 1 absorbs light with $\lambda_{\max }$ of $600 \mathrm{~nm}$ in $\mathrm{MeOH}\left(\varepsilon=8.6 \times 10^{4} \mathrm{M}^{-}\right.$

$\left.{ }^{1} \mathrm{~cm}^{-1}\right)$ and selenorhodamine 3 absorbs light with $\lambda_{\max }$ of $568 \mathrm{~nm}$ in $\mathrm{MeOH}(\varepsilon=1.03 \times$ $\left.10^{5} \mathrm{M}^{-1} \mathrm{~cm}^{-1}\right)$. In collaboration with Mike Detty of the University at Buffalo, the quantum yield for ${ }^{1} \mathrm{O}_{2}$ generation $\left[\Phi\left({ }^{1} \mathrm{O}_{2}\right)\right]$ for $\mathbf{1}$ is $0.75 \pm 0.03^{11}$ and $\Phi\left({ }^{1} \mathrm{O}_{2}\right)$ for selenorhodamine $\mathbf{3}$ is $0.85 \pm 0.03$ as determined by time-resolved spectroscopy of ${ }^{1} \mathrm{O}_{2}$ phosphorescence. ${ }^{12}$

Values of $\Phi\left({ }^{1} \mathrm{O}_{2}\right)$ are related to quantum yields for triplet generation $\left(\Phi_{\mathrm{T}}\right)$ and, based strictly on spin-orbit effects, the value of $\Phi_{\mathrm{T}}$ for $\mathbf{1}$ containing the heavier Te atom (filled $4 \mathrm{~d}$ shell) would be expected to be greater than that of $\mathbf{3}$ containing the lighter Se atom (filled $3 \mathrm{~d}$ shell). ${ }^{21}$ However, the lifetime of the triplet state $\left(\tau_{\mathrm{T}}\right)$ for Te-containing chromophores is shorter than $\tau_{\mathrm{T}}$ for comparable Se-containing dyes and relaxation of the triplet by pathways other than quenching with oxygen become competitive (i.e., phosphorescence) in air-saturated solutions. ${ }^{22}$ The fraction of triplets producing ${ }^{1} \mathrm{O}_{2}$ can 
be reduced as triplet lifetimes become shorter and the smaller value of $\Phi\left({ }^{1} \mathrm{O}_{2}\right)$ for $\mathbf{1}$ relative to $\mathbf{3}$ is certainly consistent with this analysis. Furthermore, $\mathbf{1}$ reacts with ${ }^{1} \mathrm{O}_{2}$ while $\mathbf{3}$ does not. Since oxygen is required to be close to $\mathbf{1}$ to be excited, this proximity would allow the produced ${ }^{1} \mathrm{O}_{2}$ to react 1 thus the $\Phi\left({ }^{1} \mathrm{O}_{2}\right)$ would be under estimating the $\Phi_{\mathrm{T}}$

\subsubsection{Oxidations of 1 with $\mathrm{H}_{2} \mathrm{O}_{2}$}

In earlier work, Detty and coworkers examined a number of tellurides as catalysts for activation of $\mathrm{H}_{2} \mathrm{O}_{2}$ for oxidation of thiols to disulfides. ${ }^{15,16}$ Rate constants for oxidation of the tellurides to the corresponding telluroxides with $\mathrm{H}_{2} \mathrm{O}_{2}, k_{\mathrm{H}_{2} \mathrm{O}_{2}}$, are 0.33 to $2 \mathrm{M}^{-1} \mathrm{~s}^{-1}$ for telluropyrylium compound $6^{22}$ and 3.6 to $28 \mathrm{M}^{-1} \mathrm{~s}^{-1}$ for neutral diorganotellurides 7-9 (Scheme 3.2). ${ }^{16}$ We had thought that tellurorhodamine 1 would be an excellent candidate as a catalyst for the oxidation of thiols to disulfides with $\mathrm{H}_{2} \mathrm{O}_{2}$ : $\mathbf{1}$ is oxidized to telluroxide 2 with $\mathrm{H}_{2} \mathrm{O}_{2}, 2$ is reduced by glutathione to return $\mathbf{1}$, and the 9mesityl group protects both the reduced and oxidized forms. ${ }^{11}$ However, $k_{\mathrm{H}_{2} \mathrm{O}_{2}}$ for oxidation of $\mathbf{1}$ to $\mathbf{2}$ is orders of magnitude smaller than the diorganotellurides of Scheme 3.2 .

Scheme 3.2. . Diorganotelluride catalysts for the activation of $\mathrm{H}_{2} \mathrm{O}_{2}$.

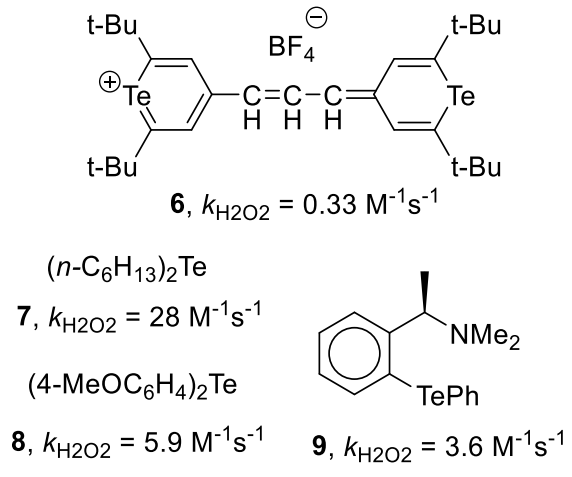


The oxidation of $1\left(1 \times 10^{-5} \mathrm{M}\right)$ to 2 with $\mathrm{H}_{2} \mathrm{O}_{2}$ in $10 \% \mathrm{H}_{2} \mathrm{O} / 90 \% \mathrm{MeOH}$ at $293 \mathrm{~K}$ was followed by UV-Vis spectroscopy as shown in Figure 3.1. Reaction with $0.05 \mathrm{M}$ $\mathrm{H}_{2} \mathrm{O}_{2}$ was complete after $4800 \mathrm{~s}$ and telluroxide 2 was identified as the product of oxidation (Figure 3.1a). ${ }^{11}$ The rate of appearance of $\mathbf{2}$ was followed at $680 \mathrm{~nm}$ as a function of time for three concentrations of $\mathrm{H}_{2} \mathrm{O}_{2}(0.01,0.03$, and $0.05 \mathrm{M})$. Typical firstorder plots of the data for the three $\mathrm{H}_{2} \mathrm{O}_{2}$ concentrations are shown in Figure 3.1b. A plot of the pseudo first-order rate constants as a function of $\mathrm{H}_{2} \mathrm{O}_{2}$ concentration is shown in Figure 3.1c. For the oxidation of 1 with $\mathrm{H}_{2} \mathrm{O}_{2}, k_{\mathrm{H}_{2} \mathrm{O}_{2}}$ in $90 \%$ aqueous $\mathrm{MeOH}$ at $293 \mathrm{~K}$ is $(1.1 \pm 0.01) \times 10^{-2} \mathrm{M}^{-1} \mathrm{~s}^{-1}$, which is 30-fold smaller than $k_{\mathrm{H}_{2} \mathrm{O}_{2}}$ for $\mathbf{6}$ and 2500-fold smaller than $k_{\mathrm{H}_{2} \mathrm{O}_{2}}$ for $\mathbf{7}$ under similar conditions. For comparison, selenorhodamine $\mathbf{3}$ does not react with $0.05 \mathrm{M} \mathrm{H}_{2} \mathrm{O}_{2}$ in $90 \%$ aqueous $\mathrm{MeOH}$ over a 2-h time period. 

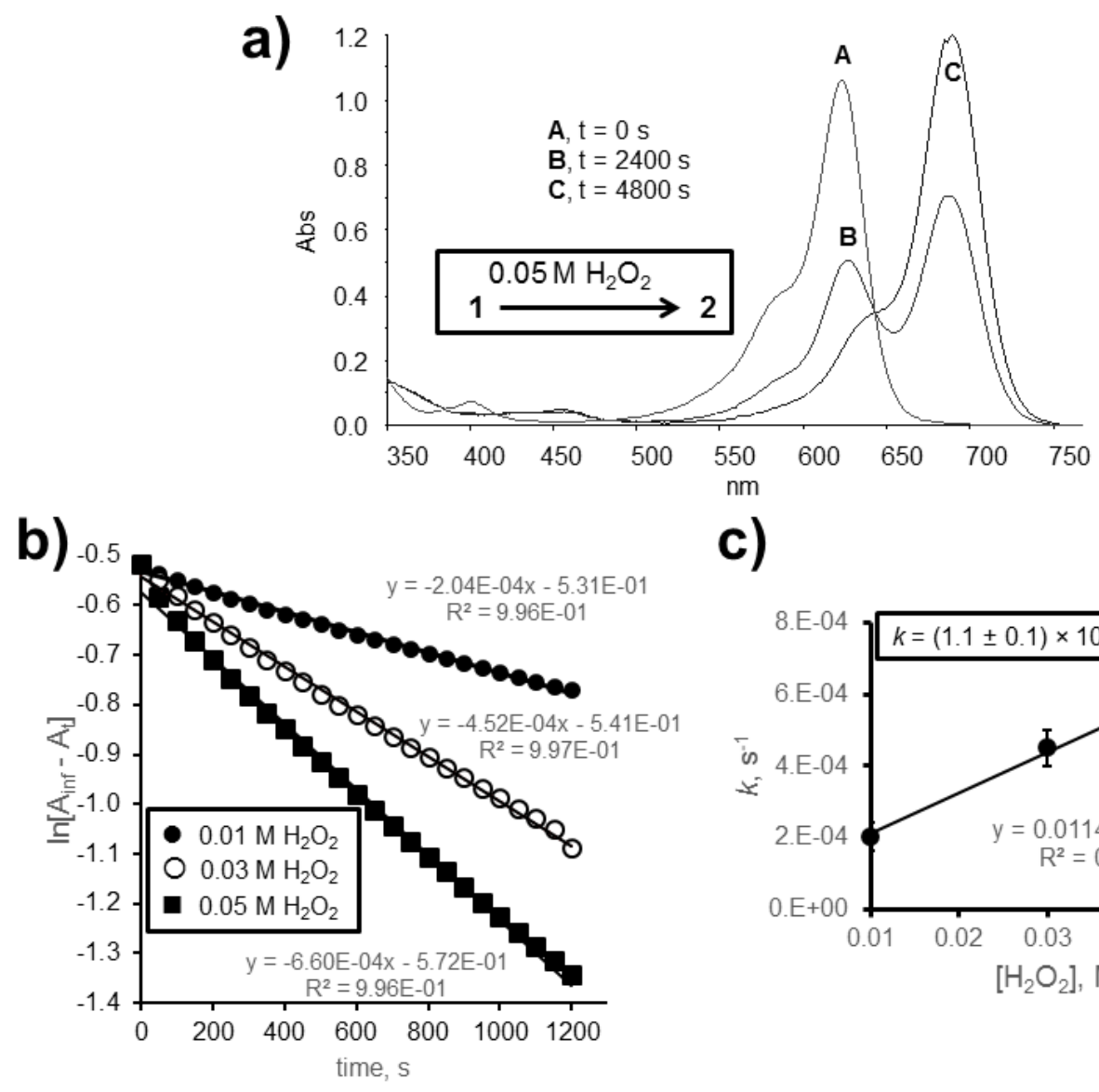

c)

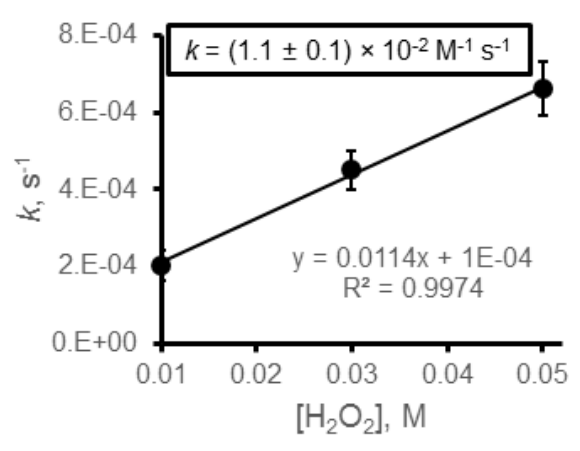

Figure 3.1. Oxidation of a $1 \times 10^{-5} \mathrm{M}$ solution of $\mathbf{1}$ with $\mathrm{H}_{2} \mathrm{O}_{2}$ in $10 \% \mathrm{H}_{2} \mathrm{O} / 90 \%$ $\mathrm{MeOH}$ to give telluroxide 2 at $293 \mathrm{~K}$. (a) Spectral changes for the conversion of $\mathbf{1}$ to 2 with $0.05 \mathrm{M} \mathrm{H}_{2} \mathrm{O}_{2}$. (b) Typical first-order plots for the appearance of 2 monitored at 680 $\mathrm{nm}$ at $0.01,0.03$, and $0.05 \mathrm{M} \mathrm{H}_{2} \mathrm{O}_{2}$. (c) A plot of mean pseudo first-order rate constants from triplicate runs as a function of $\mathrm{H}_{2} \mathrm{O}_{2}$ concentration. Error bars represent \pm 1 standard deviation. 

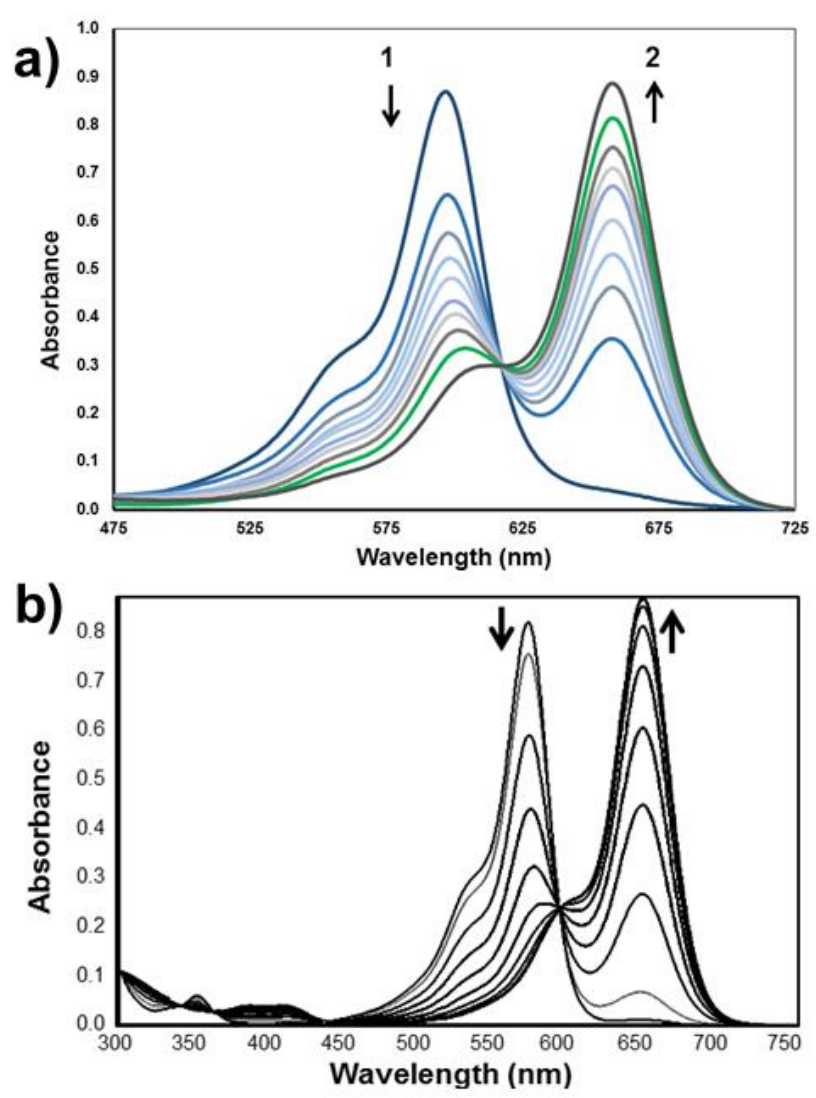

Figure 3.2. Photo-oxidation of a $1 \times 10^{-5} \mathrm{M}$ solution of $\mathbf{1}$ to give telluroxide $\mathbf{2}$ irradiated with $24 \mathrm{~W}$ white LEDs (a) in $10 \% \mathrm{H}_{2} \mathrm{O} / 90 \% \mathrm{MeOH}$ (spectrum taken every 2 min for $18 \mathrm{~min}$ ) and (b) in air-saturated $\mathrm{CDCl}_{3}$ containing $2.5 \%$ water (spectrum taken every 2 min for $16 \mathrm{~min}$ ).

\subsubsection{Photochemical reactivity of chalcogenorhodamine dyes 1 and 3}

The facile self-sensitized photo-oxidation of tellurorohodamine 1 to 2 ( $\lambda_{\max } 664$ $\mathrm{nm}$ ) in aqueous buffer or $\mathrm{MeOH}$ followed by reversible reduction back to $\mathbf{1}$ with glutathione ${ }^{11}$ suggests that $\mathbf{1}$ may be an excellent photocatalyst for the oxidation of thiols to disulfides. We next examined the photochemical reactivity of $\mathbf{1}$ and the seleniumcontaining compound, $\mathbf{3}$, under a range of conditions. 
Solutions of either $\mathbf{1}$ or $\mathbf{3}\left(1 \times 10^{-5} \mathrm{M}\right)$ in degassed solutions of $\mathrm{MeOH}$ or $\mathrm{CDCl}_{3}$ under a $\mathrm{N}_{2}$ atmosphere showed less than 5\% bleaching upon irradiation with white LEDs (24 Watt) over a 2-h time period. Irradiation of a $1 \times 10^{-5} \mathrm{M}$ solution of $\mathbf{1}$ in air-saturated $10 \% \mathrm{H}_{2} \mathrm{O} / 90 \% \mathrm{MeOH}$ with the white LEDs gave photo-oxidation to telluroxide $\mathbf{2}$ over an 18-min period with an isosbestic point at $616 \mathrm{~nm}$ (Figure 3.2a). In contrast, no loss of the chromophore of 3 in air-saturated $10 \% \mathrm{H}_{2} \mathrm{O} / 90 \% \mathrm{MeOH}$ was observed with irradiation with the white LEDs over a 2-h time period.

Irradiation of a $1 \times 10^{-5} \mathrm{M}$ solution of $\mathbf{1}$ in air-saturated dry $\mathrm{CDCl}_{3}$ gave a different outcome. After 30-s of irradiation, the appearance of 2 at $650 \mathrm{~nm}$ was observed, but $\mathbf{2}$ was not stable to the reaction conditions. The ability of the telluroxide $\mathrm{O}$ atom in tellurorhodamine telluroxides to act as an inter- or intramolecular nucleophile has been previously described. ${ }^{11}$ The addition of $2.5 \%$ water to the $\mathrm{CDCl}_{3}$ solution prior to irradiation increased the stability of $\mathbf{2}$ and photo-oxidation of $\mathbf{1}$ to $\mathbf{2}$ was observed with an isosbestic point at $598 \mathrm{~nm}$ (Figure $3.2 \mathrm{~b}$ ). 


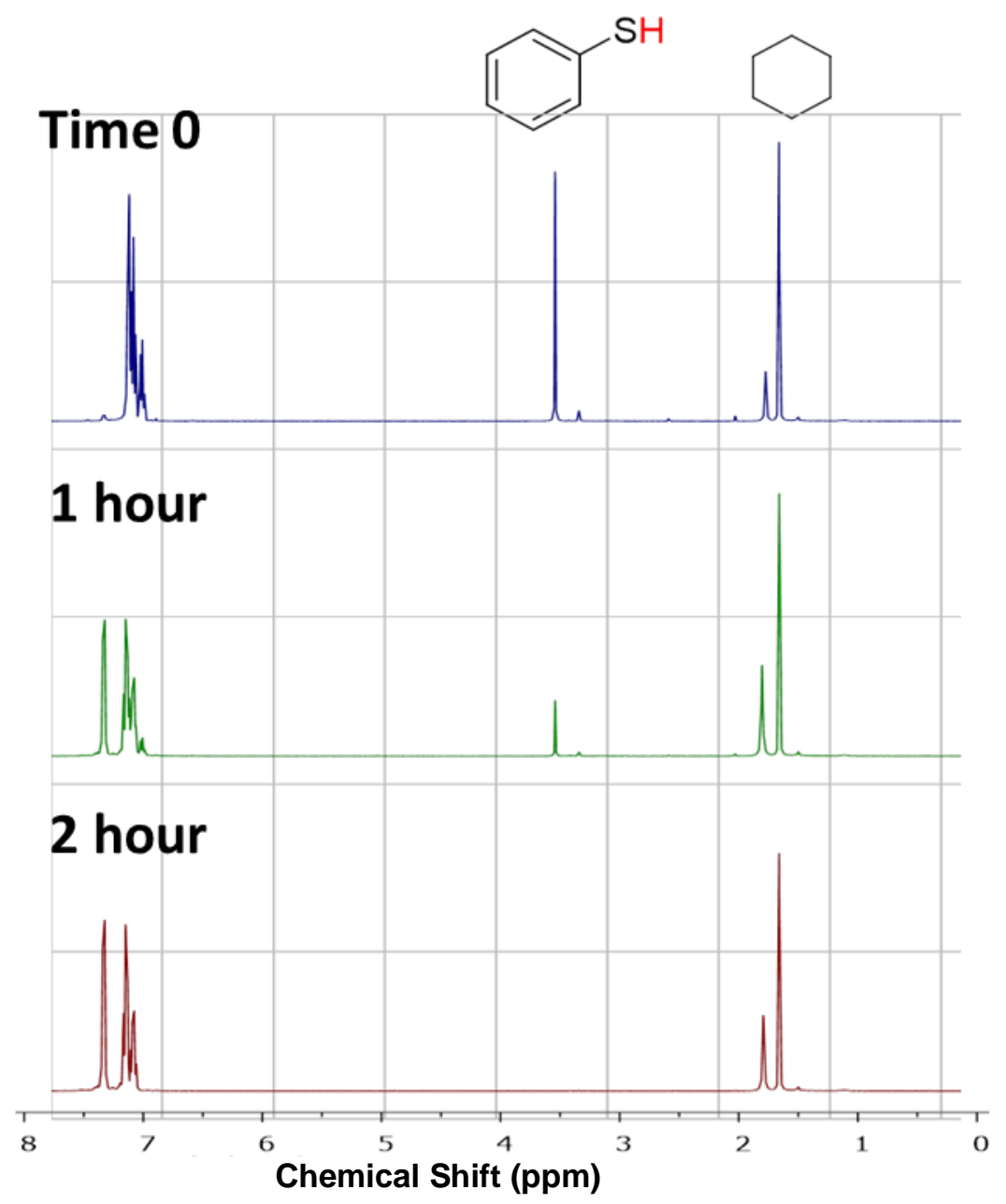

Figure 3.3. ${ }^{1} \mathrm{H}$ NMR spectra following the photo-oxidation of thiophenol with 1 mol-\% of 1 under visible light irradiation in $\mathrm{CDCl}_{3}$ containing $2.5 \%$ water at $0,1 \mathrm{~h}$, and 2 $\mathrm{h}$. The thiol proton signal at $\sim 3.5 \mathrm{ppm}$ was used to monitor reaction progress.

\subsubsection{Photocatalytic thiol oxidation}

Photocatalytic oxidations of aliphatic and aromatic thiols to disulfides were monitored by ${ }^{1} \mathrm{H}$ NMR spectroscopy using $\mathrm{CDCl}_{3}$ containing $2.5 \% \mathrm{H}_{2} \mathrm{O}$ by volume as solvent and 1 mol- $\%$ of tellurorhodamine $\mathbf{1}$ or selenorhodamine $\mathbf{3}$ (relative to thiol) as the photocatalyst. The reaction mixtures were irradiated with white LEDs under ambient conditions. Results are summarized in Table 3.1. Thiophenol and 2-naphthalenethiol 
were completely oxidized to disulfides (Table 3.1, entries 1 and 3 , respectively) within 2 $\mathrm{h}$ as shown in Figure 3.3 for the oxidation of thiophenol. Oxidation was selective as no other products were apparent upon completion of reaction.

Table 3.1. Oxidation of aromatic and aliphatic thiols $\left(5 \times 10^{-2} \mathrm{M}\right)$ to disulfides with 1 mol- $\% 1$ or $3\left(5 \times 10^{-4} \mathrm{M}\right)$ under aerobic conditions in $2.5 \% \mathrm{H}_{2} \mathrm{O} / \mathrm{CDCl}_{3}$

\begin{tabular}{ccccc}
\hline entry & $\begin{array}{c}\text { photo- } \\
\text { catalyst }\end{array}$ & thiol & $\begin{array}{c}\text { irradiation } \\
\text { time, (h) }\end{array}$ & $\begin{array}{c}\text { disulfide } \% \\
\text { conversion }^{a}\end{array}$ \\
\hline 1 & $\mathbf{1}$ & thiophenol & 2 & $\geq 99$ \\
2 & $\mathbf{3}$ & thiophenol & 3 & 5 \\
3 & $\mathbf{1}$ & 2-naphthalenethiol & 2 & $\geq 99$ \\
4 & $\mathbf{3}$ & 2-naphthalenethiol & 2 & 6 \\
5 & $\mathbf{1}$ & 2,6-dichlorothiophenol & 2 & 52 \\
$6^{b}$ & $\mathbf{1}$ & tert-butylthiol & 2 & 38 \\
$7^{b}$ & $\mathbf{1}$ & 1-dodecanethiol & 2 & 25
\end{tabular}

${ }^{a}$ Determined by ${ }^{1} \mathrm{H}$ NMR spectroscopy. ${ }^{b}$ Loss of $\mathbf{1}$ was observed with time.

The completion of thiol oxidation was indicated by a colorimetric change in the appearance of the reaction mixture. The persistence of $\mathbf{2}-$ i.e., $\mathbf{2}$ no longer being reduced to $\mathbf{1}$ - was visually identifiable by the bathochromic absorbance shift resulting in a change from a blue to a green solution. After complete conversion of thiol to disulfide, the addition of more thiol resulted in the immediate reduction of $\mathbf{2}$ to $\mathbf{1}$ and further thiol oxidation was observed with continued irradiation with visible light.

As described above, irradiation of degassed solutions of $\mathbf{1}$ under a nitrogen atmosphere in either $\mathrm{CDCl}_{3}$ or $\mathrm{MeOH}$ did not give 2. Similarly, irradiation of degassed solutions of $\mathbf{1}$ and thiophenol did not give diphenyl disulfide. These data are consistent with $\mathrm{O}_{2}$ being an integral component of the photocatalysis. 
Using selenorhodamine $\mathbf{3}$ as the photocatalyst with thiophenol (Table 3.1, entry 2) and 2-naphthalenethiol (Table 3.1, entry 4) resulted in conversions to disulfide of $\leq 6 \%$ after 2-3 h of irradiation. The observed conversion is likely the result of thiol oxidation by ${ }^{1} \mathrm{O}_{2}$ produced from irradiating the solution rather than $\mathbf{3}$ acting as a photocatalyst. No color change and no loss of absorbance of $\mathbf{3}$ were observed by UV-Vis absorption spectroscopy during irradiation indicating that $\mathbf{3}$ was not lost during the reaction and does not produce observable amounts of selenoxide. Similarly, irradiation of degassed solutions of $\mathbf{3}$ and thiophenol did not give diphenyl disulfide.

Oxidation of 2,6-dichlorothiophenol was slower than the oxidation of either thiophenol or 2-naphthalenethiol as nearly half the initial thiol concentration remained after $2 \mathrm{~h}$ (Table 3.1, entry 5). Catalyst degradation was not observed, as determined by comparing the absorbance of $\mathbf{1}$ before and after irradiation for $2 \mathrm{~h}$. Oxidation of tertbutylthiol was slower still with $38 \%$ conversion after $2 \mathrm{~h}$ (Table 3.1, entry 6). Oxidation of the aliphatic 1-dodecanethiol gave only $25 \%$ oxidation after $2 \mathrm{~h}$ (Table 3.1 , entry 7 ). Oxidation of both tert-butylthiol and 1-dodecanethiol gave significant catalyst degradation after $2 \mathrm{~h}$ of irradiation as observed spectrophotometrically by loss of $\mathbf{1}$.

\subsubsection{Reduction of telluroxide 2 with aromatic and aliphatic thiols}

The reduction of telluroxide $\mathbf{2}$ to tellurorhodamine $\mathbf{1}$ was examined with thiophenol, 2-naphthalenethiol, 2,6-dichlorothiophenol, and 1-dodecanethiol as reductants using stopped-flow spectroscopy. A solution of $2\left(5 \times 10^{-6} \mathrm{M}\right)$ for the stopped-flow studies was prepared by dissolving 1 in $10 \%$ water/90\% $\mathrm{MeOH}$ and photo-oxidizing the solution to produce 2 . 
The rates of reduction of 2 under pseudo first-order conditions using 100 equivalents of thiol $\left(5 \times 10^{-4} \mathrm{M}\right)$ were followed by stopped-flow spectroscopy monitoring the change in absorbance of $\mathbf{2}$ at $655 \mathrm{~nm}$ as shown in Figure 3.4. 2-Naphthalenethiol, thiophenol, and 2,6-dichlorothiophenol gave pseudo first-order rate constants of $1.46 \pm$ $0.08 \mathrm{~s}^{-1}, 1.06 \pm 0.06 \mathrm{~s}^{-1}$, and $0.808 \pm 0.007 \mathrm{~s}^{-1}$, respectively.
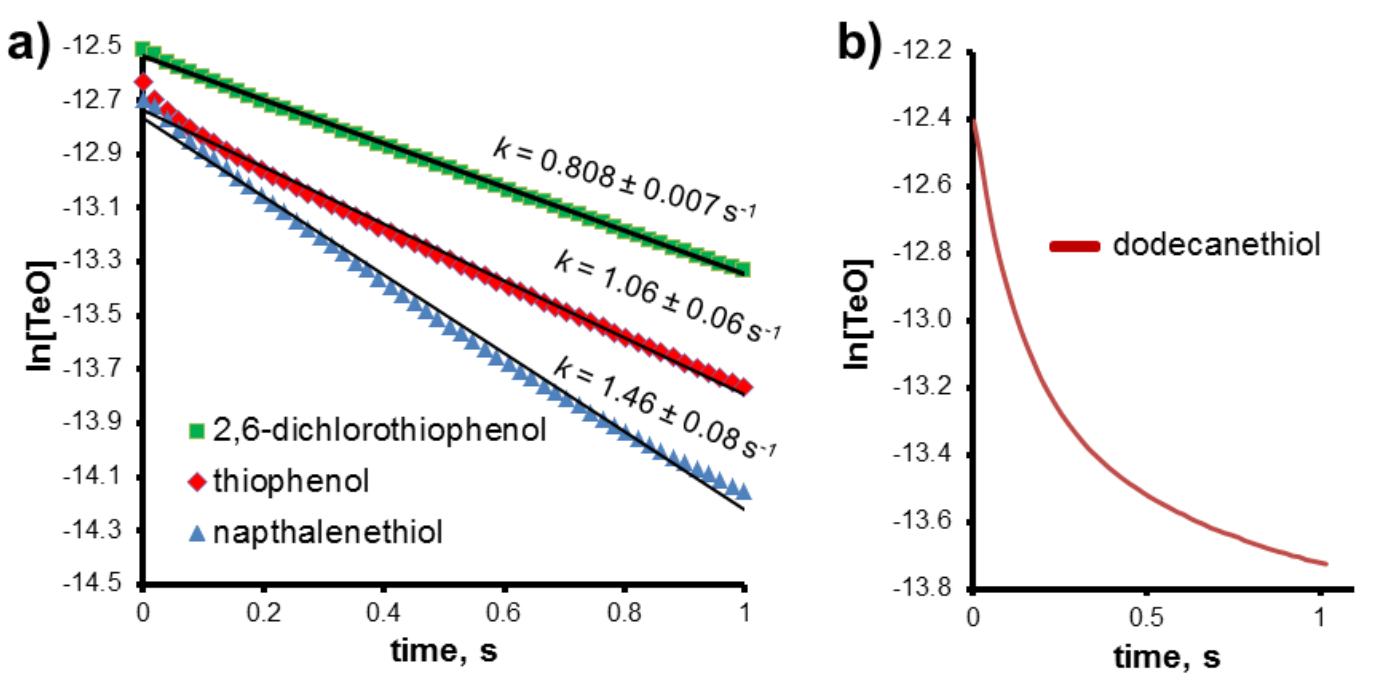

Figure 3.4. Reduction of $\mathbf{2}$ to $\mathbf{1}$ as monitored by stopped-flow spectroscopy from the diminishing of the telluroxide peak at $655 \mathrm{~nm}$ under pseudo first-order conditions a) by 2,6-dichlorothiophenol (green squares), thiophenol (red diamonds), and 2naphthalenethiol (blue triangles) and b) by 1-dodecanethiol.

If the pseudo first-order rate constants are divided by the thiol concentration $(5 \times$ $10^{-4} \mathrm{M}$ ), approximate second-order rate constants are obtained for 2-naphthalenethiol, thiophenol, and 2,6-dichlorothiophenol of $2.9 \times 10^{3} \mathrm{M}^{-1} \mathrm{~s}^{-1}, 2.1 \times 10^{3} \mathrm{M}^{-1} \mathrm{~s}^{-1}$, and $1.6 \times$ $10^{3} \mathrm{M}^{-1} \mathrm{~s}^{-1}$, respectively. Initial rates for the oxidation of thiophenol to diphenyl disulfide with $\mathrm{H}_{2} \mathrm{O}_{2}$ in aqueous $\mathrm{MeOH}$ have been reported. ${ }^{23}$ Based on an initial rate of $(1.5 \pm 0.4)$ $\times 10^{-7} \mathrm{M}^{-1} \mathrm{~min}^{-1}$ for the appearance of diphenyl disulfide with $1.0 \times 10^{-3} \mathrm{M}$ thiophenol and 
$3.75 \times 10^{-3} \mathrm{M} \mathrm{H}_{2} \mathrm{O}_{2}$, a second-order rate constant of $(6.7 \pm 1.7) \times 10^{-4} \mathrm{M}^{-1} \mathrm{~s}^{-1}$ is calculated. The rate constant for thiophenol oxidation with $\mathrm{H}_{2} \mathrm{O}_{2}$ is $\sim 10^{-6}$ that of thiophenol oxidation with $\mathbf{2}$ suggesting oxidation with produced hydrogen peroxide is not significantly contributing to thiol oxidation.

The slower rate of reduction of $\mathbf{2}$ with 2,6-dichlorothiophenol is consistent with the $52 \%$ yield of disulfide after $2 \mathrm{~h}$ (Table 3.1, entry 4). The slower rate of reduction is likely a result of the electron-withdrawing chlorine groups decreasing the nucleophilicity of the thiol S atom.

A solution of 2 was titrated with thiophenol, 2-naphthalenethiol, or 2,6dichlorothiophenol in order to determine the number of molar equivalents of thiol required for complete conversion back to $\mathbf{1}$. Two molar equivalents of aromatic thiol proved sufficient for complete reduction of $\mathbf{2}$ to $\mathbf{1}$ as determined by UV-Vis spectroscopy. 


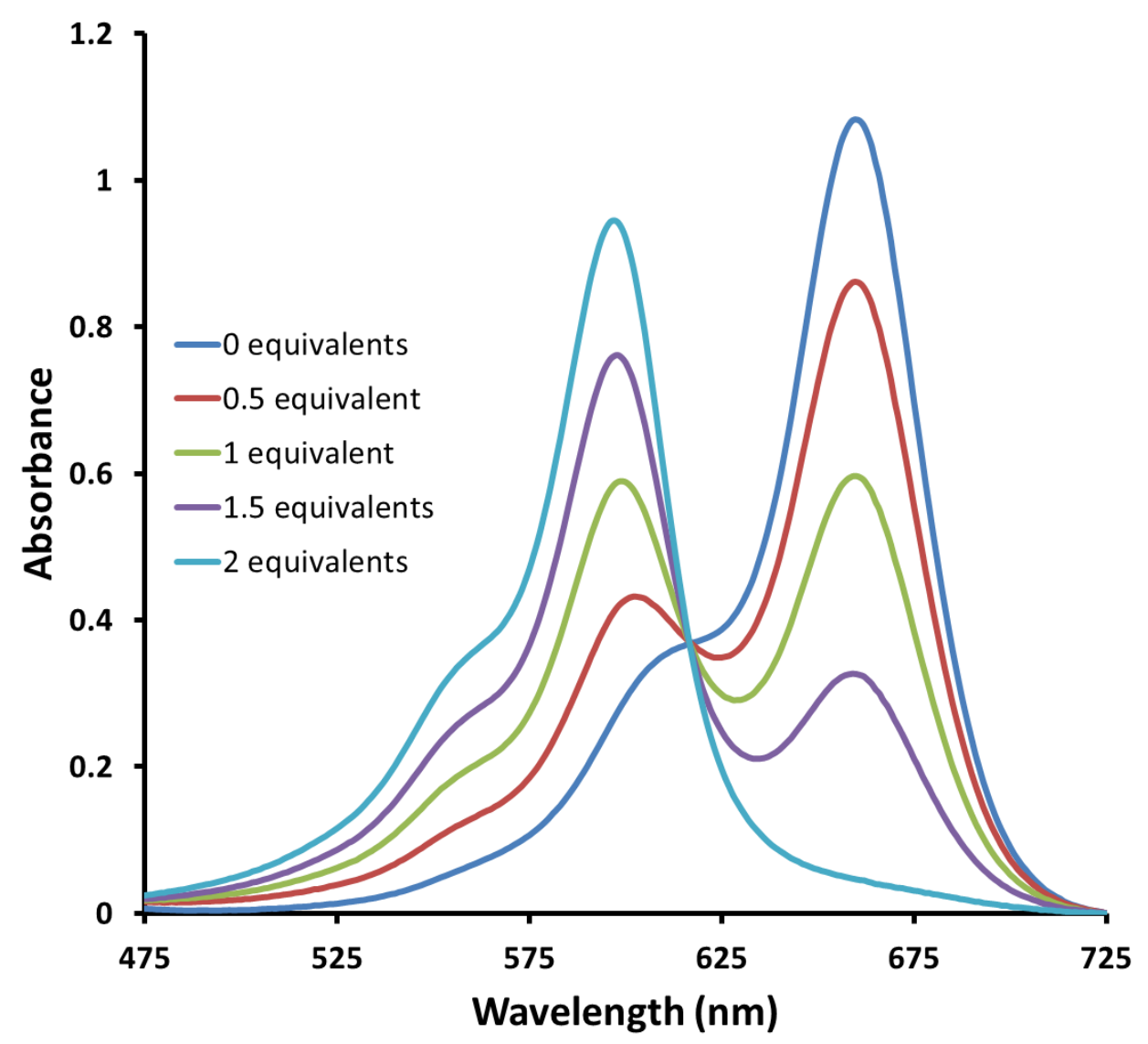

Figure 3.5. Reduction of $2\left(1 \times 10^{-5} \mathrm{M}\right)$ to 1 using 2 equivalents of thiophenol in $\mathrm{MeOH}$. In contrast to reactions with aromatic thiols, reduction of 2 using excess 1dodecanethiol under pseudo first-order conditions did not result in a first-order loss of $\mathbf{2}$, which is indicative of a competing reaction. X-ray crystal structures in earlier studies documented intramolecular and bimolecular addition of nucleophiles to the 9-position of the tellurorhodamine telluroxides, which disrupts the cyanine-like chromophore. ${ }^{11}$ Similar chemistry with 1-dodecanethiol would lead to loss of catalyst. Although the 9mesityl group sterically protects telluroxide 2 from nucleophilic addition to the 9-position during reduction with two equivalents of glutathione, ${ }^{11}$ addition of an excess of 1 - 
dodecanethiol to telluroxide $\mathbf{2}$ appears to result in a degradation pathway that is competitive with reduction of $\mathbf{2}$ to $\mathbf{1}$.

\subsubsection{Photo-oxidation of 1 to 2}

The mechanism of photo-oxidation of $\mathbf{1}$ to $\mathbf{2}$ was examined both computationally and experimentally. The data are consistent with the mechanism proposed in Scheme 3.3. The initial formation of the pertelluroxide intermediate $\mathbf{A}$ of Scheme 3.3 has been proposed as the starting point for addition of ${ }^{1} \mathrm{O}_{2}$ to diorganotellurides. ${ }^{6}$ The pertelluroxide A can oxidize a second diorganotelluride (i.e., a second molecule of 1) leading to reduction of $\mathbf{A}$ to the telluroxide (i.e., 2) and oxidation of the diorganotelluride to the telluroxide (i.e., 2). The stoichiometry of this chemistry was established in flash photolysis studies of telluropyrylium dye 6 in $\mathrm{MeOH}$ (Chart 1). The reversible addition of $\mathrm{H}_{2} \mathrm{O}$ to a diorganotelluroxide $\mathbf{B}$ forms the (dihydroxy)tellurane $\mathbf{C}{ }^{22,25}$

Irradiation of $\mathbf{1}$ in the presence of water facilitated telluroxide formation. In addition, photo-oxidation of $1.0 \times 10^{-5} \mathrm{M} 1$ to 2 also produced $8.8 \times 10^{-6} \mathrm{M} \mathrm{H}_{2} \mathrm{O}_{2}$. Concentrations of $\mathrm{H}_{2} \mathrm{O}_{2}$ were measured by an established spectrophotometric technique using oxo[5,10,15,20-tetra(4-pyridyl)porphyrinato $]$ titanium(IV). ${ }^{20}$ Approximately one equivalent of $\mathrm{H}_{2} \mathrm{O}_{2}$ is produced upon photo-oxidation of $\mathbf{1}$ to 2 in the presence of $\mathrm{H}_{2} \mathrm{O}$, which suggests pertelluroxide oxidation of $\mathbf{1}$ to $\mathbf{2}$ is less important in the presence of water. 
Scheme 3.3. Proposed mechanism of telluride oxidations with ${ }^{1} \mathrm{O}_{2}$ in the photooxidation of 1 to 2 .

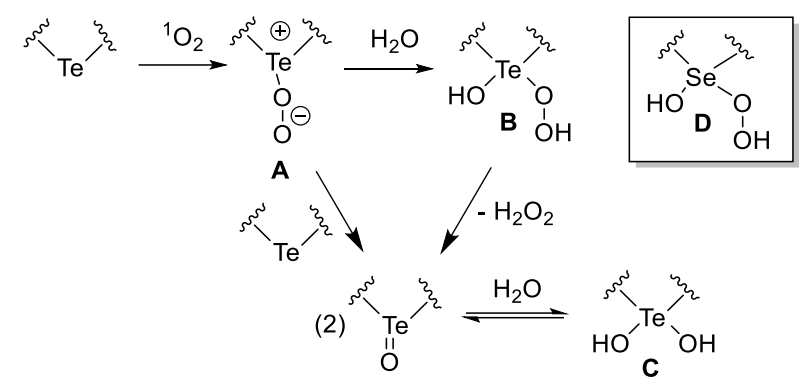

The addition of water to pertelluroxide A would form the hydroxy(perhydroxy)tellurane B. However, the addition of $\mathrm{H}_{2} \mathrm{O}_{2}$ to selenoxides gives the hydroxy(perhydroxy)selenane $\mathbf{D}$ (the selenium analogue of $\mathbf{B}$, Scheme 3.3), which are viable intermediates and have been observed by mass spectrometry and ${ }^{77} \mathrm{Se} \mathrm{NMR}$ spectroscopy. ${ }^{27}$ Loss of $\mathrm{H}_{2} \mathrm{O}_{2}$ from the hydroxy(perhydroxy)tellurane $\mathbf{B}$ forms the telluroxide. The intermediates shown in Scheme 3.3 have been studied by DFT as discussed below.

While $\mathrm{H}_{2} \mathrm{O}_{2}$ is produced upon photo-oxidation of $\mathbf{1}$ to $\mathbf{2}$ in the presence of $\mathrm{H}_{2} \mathrm{O}$, one can ask whether the presence of the thiol influences the production of $\mathrm{H}_{2} \mathrm{O}_{2}$. A solution of $1\left(5 \times 10^{-4} \mathrm{M}\right)$ and thiophenol $\left(5 \times 10^{-2} \mathrm{M}\right)$ in chloroform $(2 \mathrm{~mL})$ and water $(50 \mu \mathrm{L})$ was irradiated with white LEDs. Upon a visual change in color, from a blue to a green solution, water $(2 \mathrm{~mL})$ was added to create a biphasic solution. From the aqueous layer an aliquot $(10 \mu \mathrm{L})$ was used to determine the $\mathrm{H}_{2} \mathrm{O}_{2}$ concentration $\left(2.4 \times 10^{-4} \mathrm{M}\right)$ as described above, which corresponds to roughly half an equivalent of $\mathrm{H}_{2} \mathrm{O}_{2} \cdot{ }^{20}$ On the basis of the rates of oxidation of $\mathbf{1}$ and thiophenol described above with $\mathrm{H}_{2} \mathrm{O}_{2}$, the 
$\mathrm{H}_{2} \mathrm{O}_{2}$ produced during the photocatalysis does not compete as an oxidant with $\mathbf{2}$ for the oxidation of thiophenol to the disulfide or with ${ }^{1} \mathrm{O}_{2}$ for the oxidation of $\mathbf{1}$ to $\mathbf{2}$.

\subsubsection{DFT calculations}

Structural optimizations were performed with Gaussian 09 software ${ }^{28}$ at the B3LYP level ${ }^{29-31}$ using 6-31G(d) (C, O, S, N, H atoms) $)^{32-34}$ and LanL2DZ (Te atoms) ${ }^{35-37}$ split basis sets. ${ }^{38}$ The HOMO and LUMO molecular orbital maps of the reduced and oxidized mesityl substituted tellurorhodamine are shown in Figure 3.6.
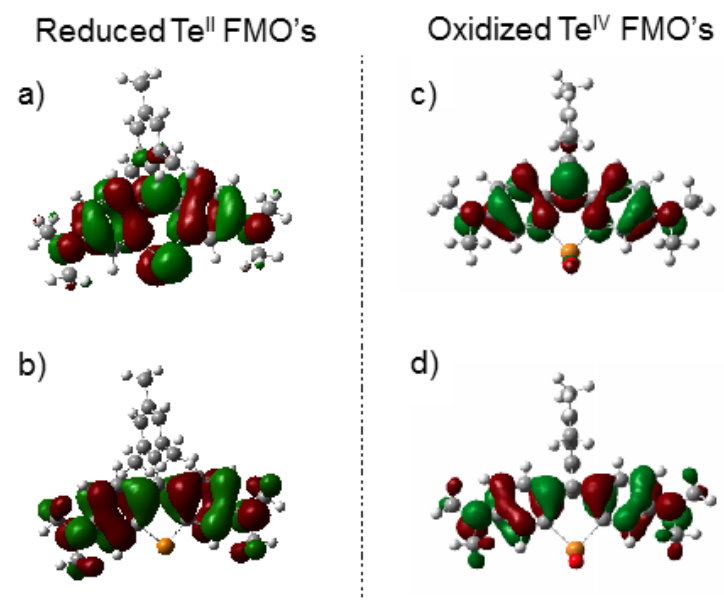

Figure 3.6. Frontier Molecular Orbitals (FMO's) from the DFT optimized geometries of the reduced tellurorhodamine $\mathbf{1}$ and oxidized $\mathbf{2}$ of the a) LUMO and b) HOMO of $\mathbf{1}$ (on the left) and the c) LUMO and d) HOMO of 2 (on the right).

The HOMO's of both the reduced species $\mathbf{1}$ and oxidized species $\mathbf{2}$ are located primarily on the $\mathrm{N}$ and $\mathrm{C}$ atoms of the 3,6-diaminoxanthylium core with minimal contributions from the Te atoms. The LUMO of the reduced species $\mathbf{1}$ has a major contribution from the Te atom which is absent in the LUMO of telluroxide 2.

Furthermore, the LUMO of the oxidized species $\mathbf{2}$ has a major contribution from the $\mathrm{C}$ 
atom in the 9-position within the xanthylium core, which supports the addition of a nucleophile at this position to give the thiol degradation from aliphatic thiols.

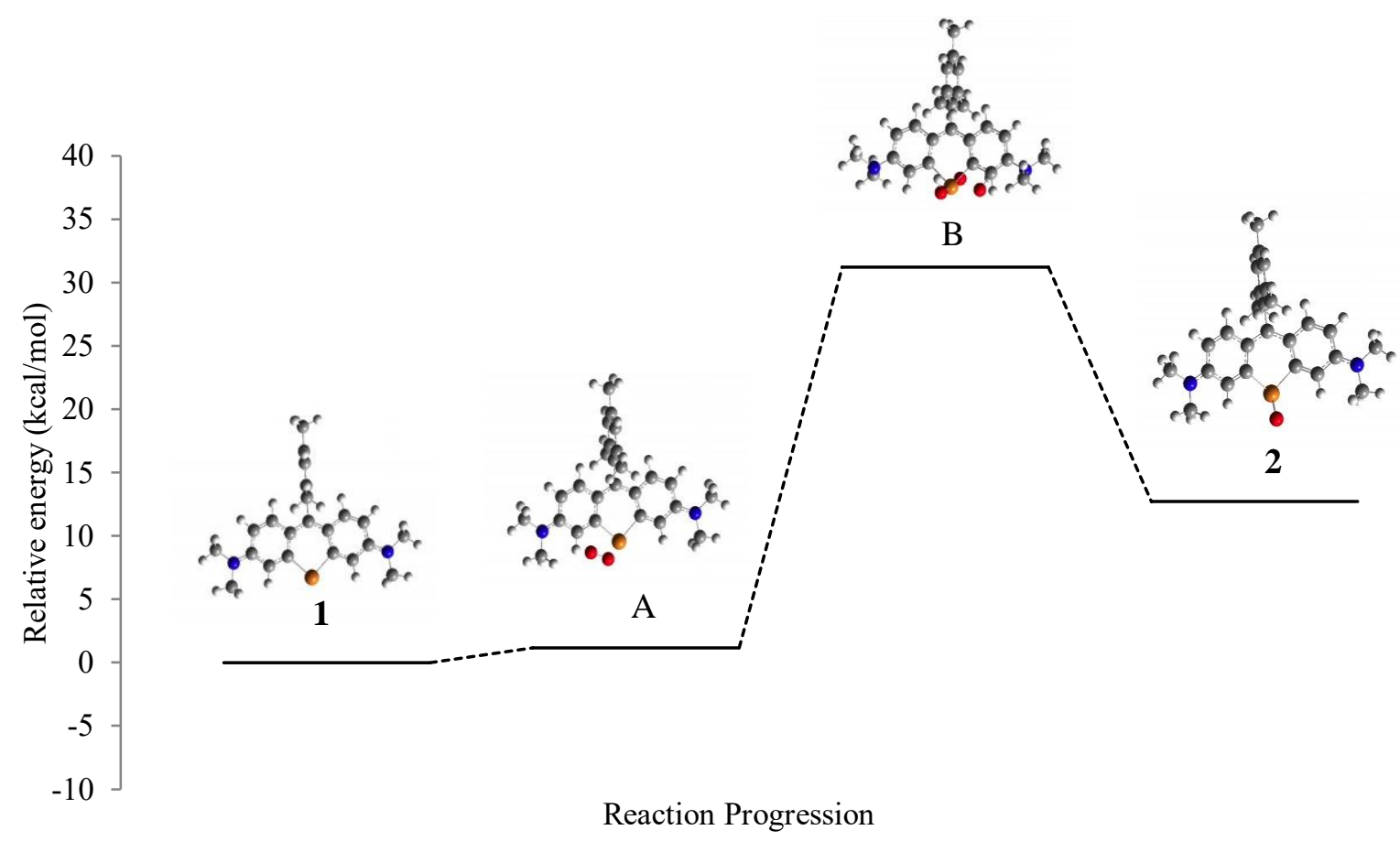

Figure 3.7. Reaction energy diagram of the formation of $\mathbf{2}$ from the reaction of $\mathbf{1}$ with ${ }^{1} \mathrm{O}_{2}$ and $\mathrm{H}_{2} \mathrm{O}$.

Reaction energy diagrams of oxidation and reduction of the telluride complex were determined using the calculated Gibb's free energies $(\Delta G)$ according to the following equation:

Equation 3.1. Calculation of Gibb's free energy

$$
\Delta \mathrm{G}=\left[\Sigma \mathrm{E}_{\text {prod }}\right]-\left[\Sigma \mathrm{E}_{\text {reac }}\right]
$$

The energy diagram of the reaction of $\mathbf{1}$ with $\mathrm{O}_{2}{ }^{1}$ to form $\mathbf{2}$ is shown in Figure 3.7. The total $\Delta \mathrm{G}$ of the oxidation of $\mathbf{1}$ to $\mathbf{2}$ with $\mathrm{O}_{2}{ }^{1}$ was calculated to be $12.7 \mathrm{kcal} / \mathrm{mol}$. The 
initial coordination of $\mathrm{O}_{2}{ }^{1}$ to the $\mathrm{Te}^{\mathrm{II}}$ of $\mathbf{1}$ to give pertelluroxide $\mathbf{A}$ (see also Scheme 4) is slightly uphill in energy $(1.17 \mathrm{kcal} / \mathrm{mol})$. Coordination of $\mathrm{H}_{2} \mathrm{O}$ followed by a proton shift forms the hydroxy(perhydroxy)tellurane $\mathbf{B}$ as a $\mathrm{Te}^{\mathrm{IV}}$ intermediate (see also Scheme 3.3). Previous to this study, the role of $\mathrm{H}_{2} \mathrm{O}$ in the oxidation mechanism was not well understood. The oxidation of $\mathbf{1}$ occurred slowly in anhydrous solvent and $\mathrm{H}_{2} \mathrm{O}$ allowed the reaction to proceed at a faster rate. We propose this specific water-coordination mechanism as a plausible explanation for the latter. The final step in the formation of $\mathbf{2}$ is elimination of $\mathrm{H}_{2} \mathrm{O}_{2}(\Delta \mathrm{G}=-18.5 \mathrm{kcal} / \mathrm{mol})$, which supports the experimentally observed results.

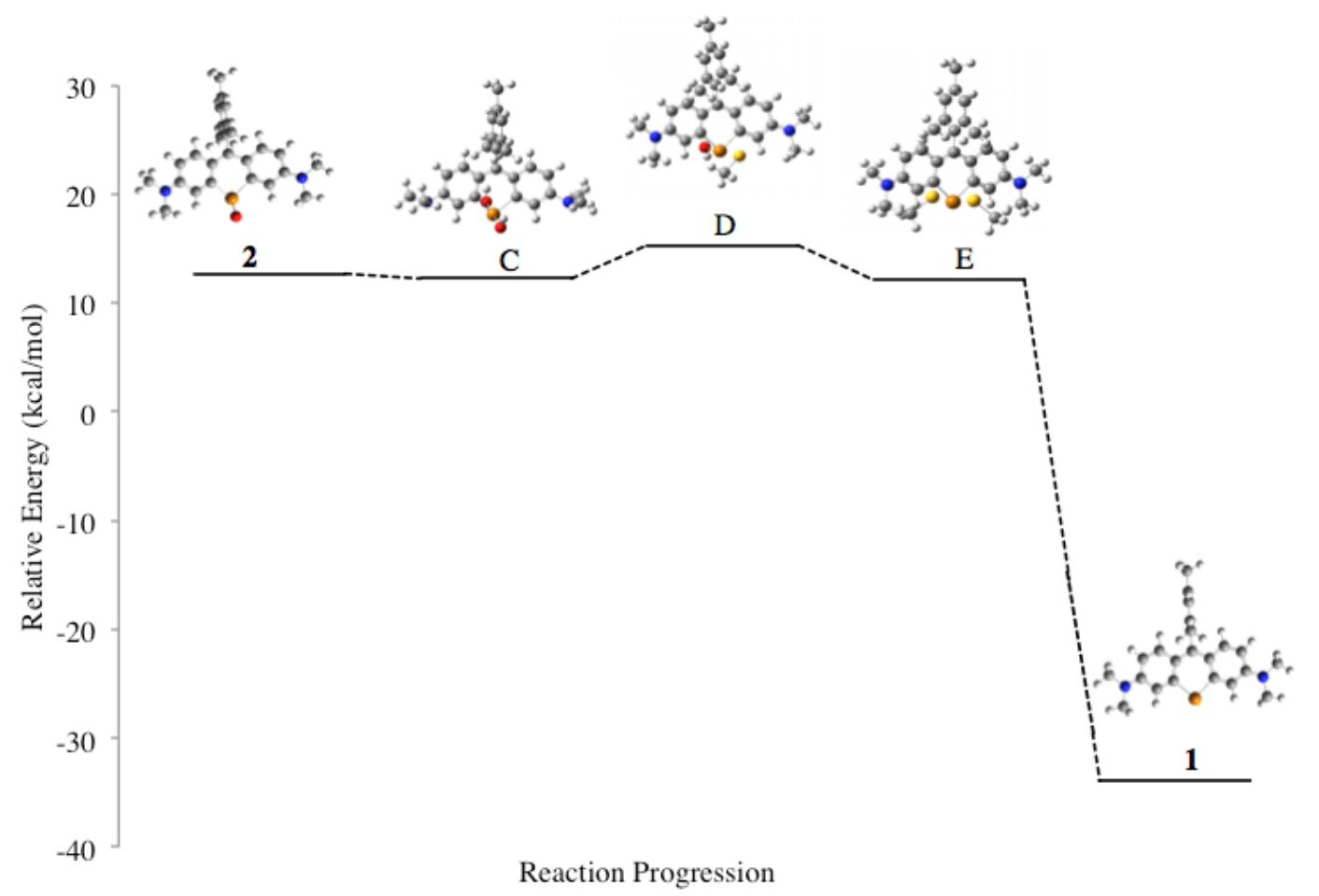

Figure 3.8. Tellurorhodamine structures in the reaction pathway for reduction of telluroxide $\mathbf{2}$ to tellurorhodamine $\mathbf{1}$ with methanethiol. Energies are relative to $\mathbf{1}$ in the balanced reaction and follow from Figure 3.7. 
To simplify the calculations, the reduction of $\mathbf{2}$ with a thiol to give $\mathbf{1}$ was calculated using methanethiol, which upon oxidation gives dimethyl disulfide. The $\Delta \mathrm{G}$ of the reduction of $\mathbf{2}$ to $\mathbf{1}$ and subsequent disulfide formation was calculated to be -46.6 $\mathrm{kcal} / \mathrm{mol}$. The computationally examined structures in Figure 3.8 were based on previously hypothesized substitution reactions, which involved the (dihydroxy)tellurane intermediate ( $\mathbf{C}$ in Scheme 3.3). ${ }^{15}$ There is a slight downhill transition from telluroxide 2 to the (dihydroxy)tellurane $\mathbf{C}(\Delta \mathrm{G}=-0.45 \mathrm{kcal} / \mathrm{mol})$, which is consistent with an equilibrium involving telluroxide 2 and $\mathrm{H}_{2} \mathrm{O} \cdot{ }^{22,25}$ Substitution of each of the hydroxy ligands with methanethiol (generating water) and subsequent reductive elimination of dimethyl disulfide reforms $\mathbf{1}$, which supports the $2: 1$ thiol to dye ratio for telluroxide reduction that was observed experimentally. The transition state for the reductive elimination is $26 \mathrm{kcal} / \mathrm{mol}$ higher than intermediate $\mathbf{E}$, and shows shortening of the $\mathrm{S}-\mathrm{S}$ bond by decreasing the S-Te-S bond angle.

\subsection{Conclusion}

Catalytic amounts of tellurorhodamine $\mathbf{1}$ were used to oxidize aromatic thiols to disulfides under aerobic conditions with visible-light irradiation. Catalytic amounts of selenorhodamine $\mathbf{3}$ with visible-light irradiation gave slower and/or poorer oxidation of thiols to disulfides. While both $\mathbf{1}$ and $\mathbf{3}$ produce ${ }^{1} \mathrm{O}_{2}$ efficiently upon irradiation, the selfsensitized generation of ${ }^{1} \mathrm{O}_{2}$ upon irradiation of $\mathbf{1}$ leads to oxidation of $\mathbf{1}$ to telluroxide $\mathbf{2}$, which is the true oxidant for the conversion of thiols to disulfides. We propose that a hydroxy(perhydroxy)tellurane is formed from the reaction of $\mathbf{1}$ with ${ }^{1} \mathrm{O}_{2}$ and water 
(structure $\mathbf{B}$ in Figure 3.7). Subsequent loss of $\mathrm{H}_{2} \mathrm{O}_{2}$ gives the telluroxide 2. Telluroxide 2 oxidizes two thiol molecules to generate a disulfide and a molecule of water, and regenerate tellurorhodamine $\mathbf{1}$, which reenters the photocatalytic cycle. The complete proposed mechanism of the catalytic cycle is summarized in Scheme 3.4. In this analysis, tellurorhodamine $\mathbf{1}$ is the resting form of the catalyst and tellurorhodamine telluroxide $\mathbf{2}$ is the active form of the catalyst. This mechanistic analysis is supported experimentally and computationally.

Scheme 3.4. Mechanism of photo-oxidation of 1 to 2 and reduction of 2 to 1 by corresponding thiol oxidation

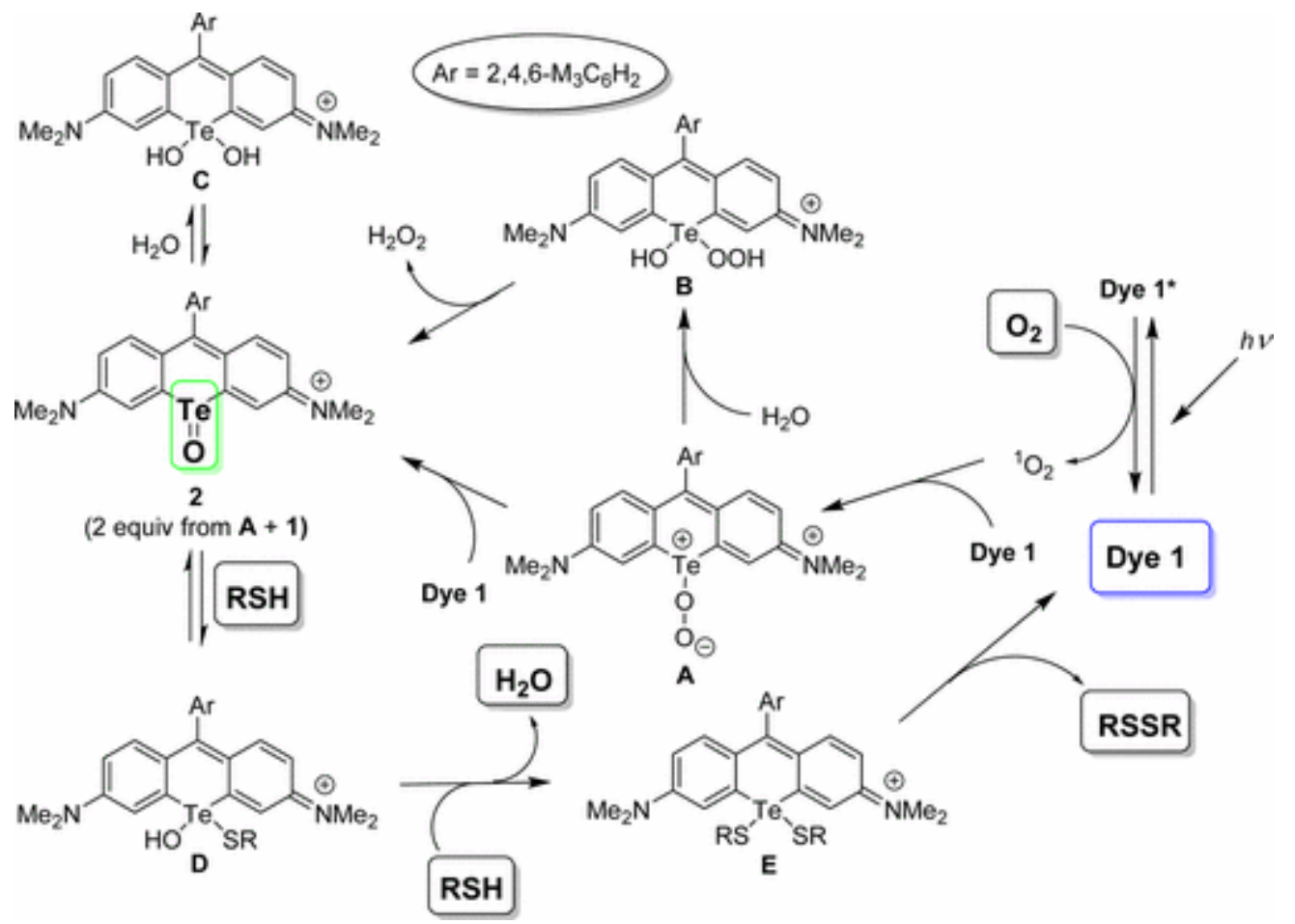

\subsection{Materials and Methods}




\subsubsection{Photo-oxidation of 1}

A solution $1\left(1 \times 10^{-5} \mathrm{M}\right)$ in $90 \% \mathrm{MeOH} / 10 \%$ water was irradiated with white LEDs and monitored by UV-Vis absorption every 2 min to give the spectra shown in Figure 2a. From this photo-oxidized sample, the $\mathrm{H}_{2} \mathrm{O}_{2}$ concentration was determined spectrophotometrically. ${ }^{26}$ A stock solution of oxo[5,10,15,20-tetra(4pyridyl)porphyrinato] titanium(IV) $\left(5 \times 10^{-6} \mathrm{M}\right)$ in perchloric acid $(2 \mathrm{M})$ was prepared. Aliquots $(10 \mu \mathrm{L})$ of the photo-oxidized sample were added to $3 \mathrm{~mL}$ of the stock solution and the change in absorption at $432 \mathrm{~nm}$ was used to determine the $\mathrm{H}_{2} \mathrm{O}_{2}$ concentration by comparing to a reference of an aliquot $(10 \mu \mathrm{L})$ of an aqueous urea peroxide solution $(1.0$ $\left.\times 10^{-5} \mathrm{M}\right)$. The samples were referenced to a blank using water $(10 \mu \mathrm{L})$. The concentration of $\mathrm{H}_{2} \mathrm{O}_{2}$ was determined to be $8.8 \times 10^{-6} \mathrm{M}(0.88$ equivalents compared to 1).

\subsubsection{Photocatalytic thiol oxidation}

Stock solutions of thiophenol, 2-naphthalenethiol, 2,6-dichlorothiophenol, and dodecane thiol $\left(1 \times 10^{-1} \mathrm{M}\right)$ were prepared in $1 \mathrm{~mL}$ of $\mathrm{CDCl}_{3}$. A stock solution of $1(1 \times$ $\left.10^{-3} \mathrm{M}\right)$ or $3\left(1 \times 10^{-3} \mathrm{M}\right)$ was prepared in $2 \mathrm{~mL}$ of $\mathrm{CDCl}_{3}$ with cyclohexane added as an internal standard. From these $0.2 \mathrm{~mL}$ of the stock solution of $\mathbf{1}$ or $\mathbf{3}$ and $0.2 \mathrm{~mL}$ of one of the thiol stock solutions were mixed in 5-mm NMR tubes resulting in a final concentration of $5 \times 10^{-2} \mathrm{M}$ of thiol and $5 \times 10^{-4} \mathrm{M}$ of $\mathbf{1}$ or $\mathbf{3}$ for each sample. A $10 \mu \mathrm{L}$ aliquot of water was added to each NMR tube. The samples were irradiated by $24-\mathrm{W}$ white LEDs with magnetic stirring (using a straightened paperclip sealed in a glass capillary) and the ${ }^{1} \mathrm{H}$ NMR spectrum was taken every $20 \mathrm{~min}$. 


\subsubsection{Reduction of 2 to 1 by aromatic thiols}

A solution of $2\left(1 \times 10^{-5} \mathrm{M}\right)$ was prepared by dissolving $\mathbf{1}$ in $95 \% \mathrm{MeOH}$ and oxidizing the solution with self-sensitized generation of ${ }^{1} \mathrm{O}_{2}$. The reduction of $\mathbf{2}$ to $\mathbf{1}$ with sequential additions of 0.5 equivalents of thiophenol, 2-naphthalenethiol, or 2,6dichlorothiophenol was followed by UV-Vis absorption spectroscopy. The addition of 2 equivalents of thiol gave complete reduction of $\mathbf{2}$ to $\mathbf{1}$.

\subsubsection{Stopped-Flow Experiments}

Stock solutions of thiophenol, 2-naphthalenethiol, 2,6-dichlorothiophenol, and dodecane thiol $\left(5 \times 10^{-4} \mathrm{M}\right)$ were prepared in $99 \% \mathrm{MeOH}$. A stock solution of $2\left(5 \times 10^{-6}\right.$ M) in $99 \% \mathrm{MeOH}$ was used for each scan. The rate of the consumption of $\mathbf{2}$ was monitored at $655 \mathrm{~nm}$ for each thiol. The acquired scans from mixing equal volumes of the stock solutions of $\mathbf{2}$ and thiol are shown as an average of triplicate measurements in Figure 3.4. The sample-handling unit was fitted with two drive syringes that are mounted inside a thermostatted-bath compartment, which allowed for variable-temperature experimentation. The optical detection cell was set up in the 10-mm path length.

\subsubsection{Determination of Singlet Oxygen Yield for Selenorhodamine}

Generation of ${ }^{1} \mathrm{O}_{2}$ was assessed by its luminescence peak at $1270 \mathrm{~nm}$. Timeresolved detection of the long-lived ${ }^{1} \mathrm{O}_{2}$ emission was used to distinguish signal from ${ }^{1} \mathrm{O}_{2}$ as previously described. ${ }^{11}$ The samples (MeOH solutions of $\mathbf{3}$ or tetramethyl selenorosamine used as a standard in quartz cuvettes) were placed in front of the spectrometer entrance slit.

\subsubsection{Computational Details}


Calculations were done with Gaussian09 ${ }^{21}$ input files and results were visualized using GaussView05. ${ }^{39}$ All structures were optimized using B3LYP ${ }^{22}$ level of theory with 6-31G $(\mathrm{d})^{32-34}$ basis set for all light atoms and LanL2DZ ${ }^{35-37}$ for Te. Transition states were located with the QST2 key word. Energy values were obtained from the free energy from the frequency calculations. The HOMO and LUMO of the reduced tellurorhodamine 1 and the oxidized tellurorhodamine telluroxide $\mathbf{2}$ were obtained from the optimized structures.

\subsection{References}

1. Campbell, A. N. \& Stahl, S. S. Overcoming the "Oxidant Problem" : Strategies to Use $\mathrm{O} 2$ as the Oxidant in Organometallic C-H Oxidation Reactions Catalyzed by $\mathrm{Pd}$ (and Cu). Acc. Chem. Res. 45, 851-863 (2012).

2. Prier, C. K., Rankic, D. A. \& Macmillan, D. W. C. Visible Light Photoredox Catalysis with Transition Metal Complexes: Applications in Organic Synthesis. Chem. Rev. 113, 5322-5363 (2013).

3. Narayanam, J. M. R. \& Stephenson, C. R. J. Chem Soc Rev Visible light photoredox catalysis : applications in organic synthesis. Chem Soc Rev 40, 102113 (2011).

4. Ravelli, D., Fagnoni, M. \& Albini, A. Photoorganocatalysis. What for? Chem Soc Rev 42, 97-113 (2013).

5. Lang, X., Ma, W., Chen, C., Ji, H. \& Zhao, J. Selective aerobic oxidation mediated by TiO2 photocatalysis. Acc. Chem. Res. 47, 355-363 (2014).

6. Oba, M., Tanaka, K., Nishiyama, K. \& Ando, W. Aerobic oxidation of thiols to disulfides catalyzed by diaryl tellurides under photosensitized conditions. J. Org. Chem. 76, 4173-4177 (2011).

7. Romero, N. A. \& Nicewicz, D. A. Mechanistic Insight into the Photoredox Catalysis of Anti- Markovnikov Alkene Hydrofunctionalization Reactions. J. Am. Chem. Soc. 136, 17024-17035 (2014).

8. Margrey, K. A. \& Nicewicz, D. A. A General Approach to Catalytic Alkene AntiMarkovnikov Hydrofunctionalization Reactions via Acridinium Photoredox 
Catalysis. Acc. Chem. Res. 49, 1997-2006 (2016).

9. Condie, A. G., Gonza, C. \& Stephenson, C. R. J. Visible-Light Photoredox Catalysis : Aza-Henry Reactions via C - H Functionalization. J. Am. Chem. Soc. 132, 1464-1465 (2010).

10. Pitre, S. P., Mctiernan, C. D. \& Scaiano, J. C. Library of Cationic Organic Dyes for Visible-Light-Driven Photoredox Transformations. 66-76 (2016) doi:10.1021/acsomega.6b00058.

11. Kryman, M. W. et al. Organotellurium fluorescence probes for redox reactions: 9aryl-3,6-diaminotelluroxanthylium dyes and their telluroxides. Organometallics 32, 4321-4333 (2013).

12. Kryman, M. W. et al. Synthesis and properties of heavy chalcogen analogues of the texas reds and related rhodamines. Organometallics 33, 2628-2640 (2014).

13. Engman, L., Stern, D., Pelcman, M. \& Andersson, C. M. Thiol Peroxidase Activity of Diorganyl Tellurides. J. Org. Chem. 59, 1973-1979 (1994).

14. Vessman, K., Ekstrom, M., Berglund, M., Andersson, C. \& Engman, L. Catalytic Antioxidant Activity of Diaryl Tellurides in a Two-Phase Lipid Peroxidation Model. J. Org. Chem. 60, 4461-4467 (1995).

15. Detty, M. R., Friedman, A. E. \& Oseroff, A. R. A Mechanism for the Oxidation of Glutathione to Glutathione Disulfide with Organotellurium(IV) and Organoselenium(IV) Compounds. A Stepwise Process with Implications for Photodynamic Therapy and Other Oxidative Chemotherapy. J. Org. Chem. 82458250 (1994).

16. You, Y., Ahsan, K. \& Detty, M. R. Mechanistic Studies of the Tellurium (II)/ Tellurium (IV) Redox Cycle in Thiol Peroxidase-like Reactions of Diorganotellurides in Methanol. J. Am. Chem. Soc. 4918-4927 (2003).

17. Koide, Y. et al. A reversible near-infrared fluorescence probe for reactive oxygen species based on Te-rhodamine. Chem. Commun. 48, 3091 (2012).

18. Oba, M., Endo, M., Nishiyama, K., Ouchi, A. \& Ando, W. Photosensitized oxygenation of diaryl tellurides to telluroxides and their oxidizing properties. 2, 1672-1673 (2004).

19. Kearns, D. R. PHYSICAL AND CHEMICAL PROPERTIES OF SINGLET MOLECULAR OXYGEN. 1970, 395-427 (1971).

20. Matsubara, C., Kawamoto, N. \& Takamura, K. Oxo[5, 10, 15, 20-tetra(4pyridyl)porphyrinato]titanium(IV): an ultra-high sensitivity spectrophotometric reagent for hydrogen peroxide. Analyst 117, 1781 (1992). 
21. Frisch, M. J.; Trucks, G.W.; Schlegel, H. B.; Scuseria, G. E.; Robb, M. A.; Cheeseman, J. R.; Scalmani, G.; Barone, V.;Mennucci, B.; Petersson, G. A.; Nakatsuji, H.; Caricato, M.; Li, X.; Hratchian, H. P.; Izmaylov, A. F.; Bloino, J.; Zheng, G.; Sonnenber, D. J. Gaussian 09. Gaussian, Inc. Wallingford CT 2-3 (2009) doi:111.

22. Frisch, M. J. et al. Gaussian 09, Revision B.01; Gaussian. Inc.: Wallingford, CT 2009-2009 (2010). doi:111. 


\section{Simplification of the potassium ferrioxalate actinometer}

\subsection{Background}

The development of photocatalysts has been of great interest particularly in areas of green chemistry for organic transformations, ${ }^{1-5}$ and solar fuel production i.e. artificial photosynthesis. ${ }^{6-8}$ An important measurement of the efficiency of such photocatalysts is the quantum yield (QY), which is defined as moles of desired product per mole of photon absorbed. ${ }^{9}$ This measurement is vital in determining the applicability and scalability of a light driven process. ${ }^{10}$ Typical quantum yield values range between zero and one in which a quantum yield of one would indicate that every photon absorbed results in the desired product. Quantum yields above one indicate subsequent dark reactions after photoinitiation. The most common way of determining the quantum yield of a photocatalyst is to use a chemical actinometer, with a known quantum yield, to determine the number of absorbed photons, and take the ratio of absorbed photons to the product formed. ${ }^{11}$

The most commonly used chemical actinometer is potassium ferrioxalate. ${ }^{12-14}$ It utilizes the photoreduction of potassium ferrioxalate to produce an $\mathrm{Fe}^{2+}$ species. UV-Vis spectroscopy is used to determine the $\mathrm{Fe}^{2+}$ concentration by monitoring an $\mathrm{Fe}^{2+}$ 9, 10 phenanthroline complex. Potassium ferrioxalate can absorb photons with wavelengths between 300 and $580 \mathrm{~nm}$ to produce iron(II) oxalate and oxalate. In a subsequent dark reaction the $\mathrm{Fe}^{3+}$ oxalate is oxidized by oxalate to produce Iron (III) and a dianionic oxalate species. The $\mathrm{Fe}^{3+}$ oxalate then reduces to $\mathrm{Fe}^{2+}$ oxalate by the release of carbon 
dioxide. The reaction follows a 2:2:1 stoichiometry, as seen in Scheme 4.1, of iron (II) oxalate, carbon dioxide, and oxalate per photon, respectively. The ligand 9,10phenanthroline is then used to form a visible light absorbing iron (II) complex to determine the number of photons absorbed by the sample. ${ }^{15}$

Challenges arise with this method since to determine the amount of $\mathrm{Fe}^{2+}$ produced, Scheme 4.1. Photoreduction of potassium ferrioxalate. ${ }^{15}$

$$
\begin{aligned}
& {\left[\mathrm{Fe}^{3+}\left(\mathrm{C}_{2} \mathrm{O}_{4}\right)\right]^{3-}+h v \longrightarrow\left[\mathrm{Fe}^{2+}\left(\mathrm{C}_{2} \mathrm{O}_{4}\right)_{2}\right]^{2-}+\mathrm{C}_{2} \mathrm{O}_{4}^{-}} \\
& {\left[\mathrm{Fe}^{3+}\left(\mathrm{C}_{2} \mathrm{O}_{4}\right)\right]^{3-}+\mathrm{C}_{2} \mathrm{O}_{4}^{-} \longrightarrow\left[\mathrm{Fe}^{3+}\left(\mathrm{C}_{2} \mathrm{O}_{4}\right)_{2}\right]^{2-}+\mathrm{C}_{2} \mathrm{O}_{4}{ }^{2-}} \\
& {\left[\mathrm{Fe}^{3+}\left(\mathrm{C}_{2} \mathrm{O}_{4}\right)_{2}\right]^{2-} \longrightarrow\left[\mathrm{Fe}^{2+}\left(\mathrm{C}_{2} \mathrm{O}_{4}\right)_{2}\right]^{2-}+2 \mathrm{CO}_{2}}
\end{aligned}
$$

$$
2\left[\mathrm{Fe}^{3+}\left(\mathrm{C}_{2} \mathrm{O}_{4}\right)\right]^{3-}+h v \longrightarrow 2\left[\mathrm{Fe}^{2+}\left(\mathrm{C}_{2} \mathrm{O}_{4}\right)_{2}\right]^{2-}+2 \mathrm{CO}_{2}+\mathrm{C}_{2} \mathrm{O}_{4}{ }^{2-}
$$

aliquots must be taken away from the light source at predetermined time points and stored in the dark for 1 hour to form the visible-light absorbing phenanthroline complex. Therefore, the rates of photons per second calculated from such measurements are often derived from only a few data points. Real time monitoring of the potassium ferrioxalate photoreduction has been reported where ${ }^{13} \mathrm{C}$ NMR has been used to quantify the production of $\mathrm{CO}_{2}$ rather than $\mathrm{Fe}^{2+} .{ }^{16}$ The amount of photons absorbed could be calculated, but only for a limited time before the $\mathrm{CO}_{2}$ concentration in solution reached saturation.

Herein we describe a simplified version to monitor the photoreduction of potassium ferrioxalate in real time. The method developed focuses on determining the 
amount of carbon dioxide produced by pressure change or collection rather than spectroscopic methods. An adapted version of the pressure change method was developed by collecting the $\mathrm{CO}_{2}$ to measure a change of volume instead. The monitoring of $\mathrm{CO}_{2}$ rather than $\mathrm{Fe}^{2+}$ was advantageous as multiple measurements could be obtained in real time from only one sample rather than multiple. Also, a Fe ${ }^{2+}$ calibration curve is typically required for the traditional method and is not for this method. Here we compare the results of these methods with the traditional method.

\subsection{Results and Discussion}

\subsubsection{Traditional spectroscopic method of $\mathrm{Fe}(\mathrm{II})$ detection}

Typically the measurement of $\mathrm{Fe}^{2+}$ produced by photoreduction utilizes the absorbance of an iron (II) phenanthroline sturcture (ferroin). To quantify $\mathrm{Fe}^{2+}$ production 11 standard calibration solutions containing ferroin are prepared. Once prepared, the solutions must be placed in the dark for 1 hour before measuring the visible absorbance at $511 \mathrm{~nm}$. The resulting absorbances from 350 to $600 \mathrm{~nm}$, as seen in Figure 4.1, show $\lambda_{\max }$ at $511 \mathrm{~nm}$. The slope of the concentration vs the absorbance max at $511 \mathrm{~nm}$ can then be used to calculate the molar absorptivity of ferroin. An error in concentration in one calibration solution can decrease the correlation coefficient of the trend line enough to where the entire calibration curve needs to be repeated.

Using the calculated molar absorptivity of ferroin the total Fe(II) produced from the photolysis of potassium ferrioxalate can be calculated. Photoreduction of potassium ferrioxalate was performed in triplicate using this method. Solutions of potassium 
ferrioxalate were prepared in the dark, to avoid incident light, and then irradiated with blue LEDs. The aliquots from the solutions were then removed at designated time points (20, 40, 60 minutes). The alliquotes were diluted and phenanthroline was added to produce ferroin. After an hour in the dark, the absorbance spectrum of each sample was taken and the amount of $\mathrm{Fe}(\mathrm{II})$ was determined by Equation 4.1.

Equation 4.1. Spectroscopic calculation of Fe(II)

$$
\mathrm{n}_{\mathrm{Fe} 2+}=\frac{6.023 \times 10^{20} \mathrm{~V}_{1} \mathrm{~V}_{3} \log _{10}\left(\mathrm{I}_{0} / \mathrm{I}\right)}{\mathrm{V}_{2} \mathrm{l} \varepsilon}
$$

Where $\mathrm{V}_{1}$ is the volume of the sample solution that was irradiated $(10 \mathrm{~mL}), \mathrm{V}_{2}$ is the volume of sample taken for analysis $(0.1 \mathrm{~mL}), \mathrm{V}_{3}$ is the final volume that $\mathrm{V}_{2}$ is diluted $(100 \mathrm{~mL}), \log _{10}\left(\mathrm{I}_{0} / \mathrm{I}\right)$ is the measured absorbance of ferroin at $511 \mathrm{~nm}, 1$ is the path length of the spectrophotometer cell $(1 \mathrm{~cm})$, and $\varepsilon$ is molar extinction coefficient determined experimentally from the Fe(II) calibration curve (11000 $\left.\mathrm{L} \mathrm{mols}^{-1} \mathrm{~cm}^{-1}\right)$. 


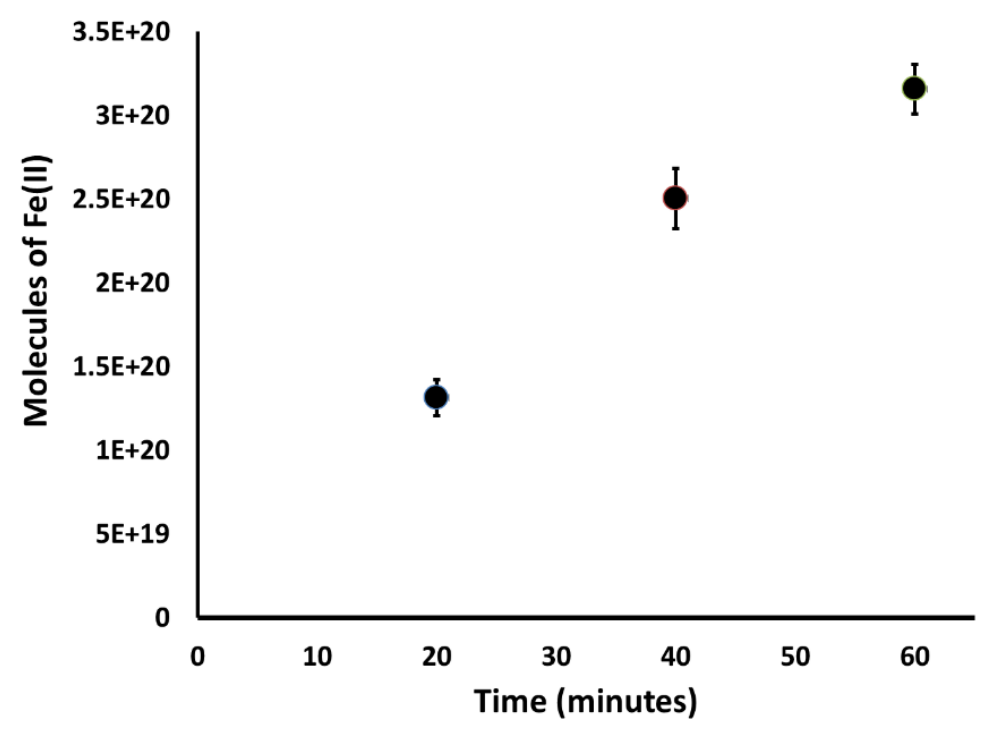

Figure 4.1. The total Fe(II) produced over time as determined spectroscopically.

Table 4.1. Selected quantum yields of the photoreduction of potassium ferrioxalate at various wavelengths. ${ }^{15}$

\begin{tabular}{ccc}
\hline Wavelength $(\mathbf{n m})$ & $\left(\mathbf{K}_{3} \mathbf{F e}\left(\mathbf{C}_{2} \mathbf{O}_{4}\right)_{3}\right) \mathbf{M}$ & $\varphi$ \\
\hline 579 & 0.15 & 0.013 \\
546 & 0.15 & 0.15 \\
480 & 0.15 & 0.94 \\
436 & 0.15 & 1.01 \\
366 & 0.006 & 1.21 \\
253 & 0.006 & 1.25 \\
\hline
\end{tabular}

The total photons absorbed can then be calculated using the Fe(II) produced and the known quantum yield of the photoreduction at the wavelength irradiated. While, the LEDs used are polychromatic light, the spectrum is narrow enough to treat it as 
monochromatic. Furthermore, the quantum yield of potassium ferrioxalate varies only slightly for wavelengths under $480 \mathrm{~nm}$. The amount of photons absorbed was calculated using equation 4.2 with a quantum yield of 0.94 for light at $480 \mathrm{~nm}$.

Equation 4.2. Calculation of photons absorbed

$$
\mathrm{n}=\frac{\mathrm{n}_{\mathrm{Fe} 2+}}{\phi_{\lambda}}
$$

The lengthy process results in a total of one data point per solution work-up. The process was repeated in triplicate using aliquots from three different irradiated samples. The average total amount of Fe(II) produced is shown with error bars representing one standard deviation from the mean as seen in Figure 4.1. Care was taken to avoid incident light through the multiple steps, however, this is especially challenging when placing the cuvette in the UV-Vis spectrometer. Time points were not taken after an hour because visible precipitation of $\mathrm{Fe}(\mathrm{II})$ occurred which would result in erroneous low measurements of Fe(II) produced. Overall, this spectroscopic method of chemical actinometry results in hours of work with several possible points to introduce error.

\subsubsection{Calculation of photons absorbed by pressure increase}

Reviewing the photoreduction of potassium ferrioxalate; it can be seen that the reaction produces 2 moles of $\mathrm{CO}_{2}$ and 1 mole of an oxalate dianion for every photon

absorbed. The production of $\mathrm{CO}_{2}$ has been previously measured in lieu of $\mathrm{Fe}^{2+}$ by ${ }^{13} \mathrm{C}$ NMR, however, the reaction progress could only be monitored until the $\mathrm{CO}_{2}$ saturated the solution and was no longer dissolved. Measuring $\mathrm{CO}_{2}$, rather than $\mathrm{Fe}^{2+}$, has distinct 
advantages, including real-time measurements not requiring the formation of the phenanthroline complex. We show that the $\mathrm{CO}_{2}$ produced may be measured by a change in pressure after saturation of the solution. The amount of $\mathrm{CO}_{2}$ produced can then be calculated using the ideal gas law in combination with Henry's law to account for the dissolved $\mathrm{CO}_{2}$ as shown in Equation 4.3. However, utilizing the slope of pressure change over time to determine the rate of photons absorbed is based on hundreds of data points rather than triplicate and relies less on the theoretical value of Henry's Law.

Equation 4.3. Calculation of moles of $\mathrm{CO}_{2}$ by change in pressure.

$$
n=\frac{R T}{P V_{1}}+\frac{P}{K_{H}} \times V_{2}
$$

Where $n$ is the number of moles of $\mathrm{CO}_{2}$ produced at a given time point, $R$ is the ideal gas law constant $\left(0.08206 \mathrm{~L} \mathrm{~atm} \mathrm{~mol}^{-1} \mathrm{~K}^{-1}\right), T$ is the temperature in Kelvin $\left(295{ }^{\circ} \mathrm{K}\right)$ which was held constant through out the experiment, $P$ is the pressure at a given time point $V_{l}$ is the total volume $(0.040 \mathrm{~L})$ of the headspace of the reaction vessel which was held constant throughout the experiment, $K_{H}$ is Henry's Law Constant for $\mathrm{CO}_{2}(29.41$ atm $\left.\mathrm{mol}^{-1}\right)$, and $V_{2}$ is the volume of the solution irradiated $(0.01 \mathrm{~L})$ which is constant throughout the experiment.

To compare the results of $\mathrm{CO}_{2}$ produced directly to the spectroscopically measured $\mathrm{Fe}^{2+}$ production, the conditions were kept identical using the same light source, reaction vessel, and solution volume. However, to determine the change in pressure over time, the solutions were sealed with caps equipped with pressure sensors and the pressure was measured every 30 seconds over the course of 8 hours. 


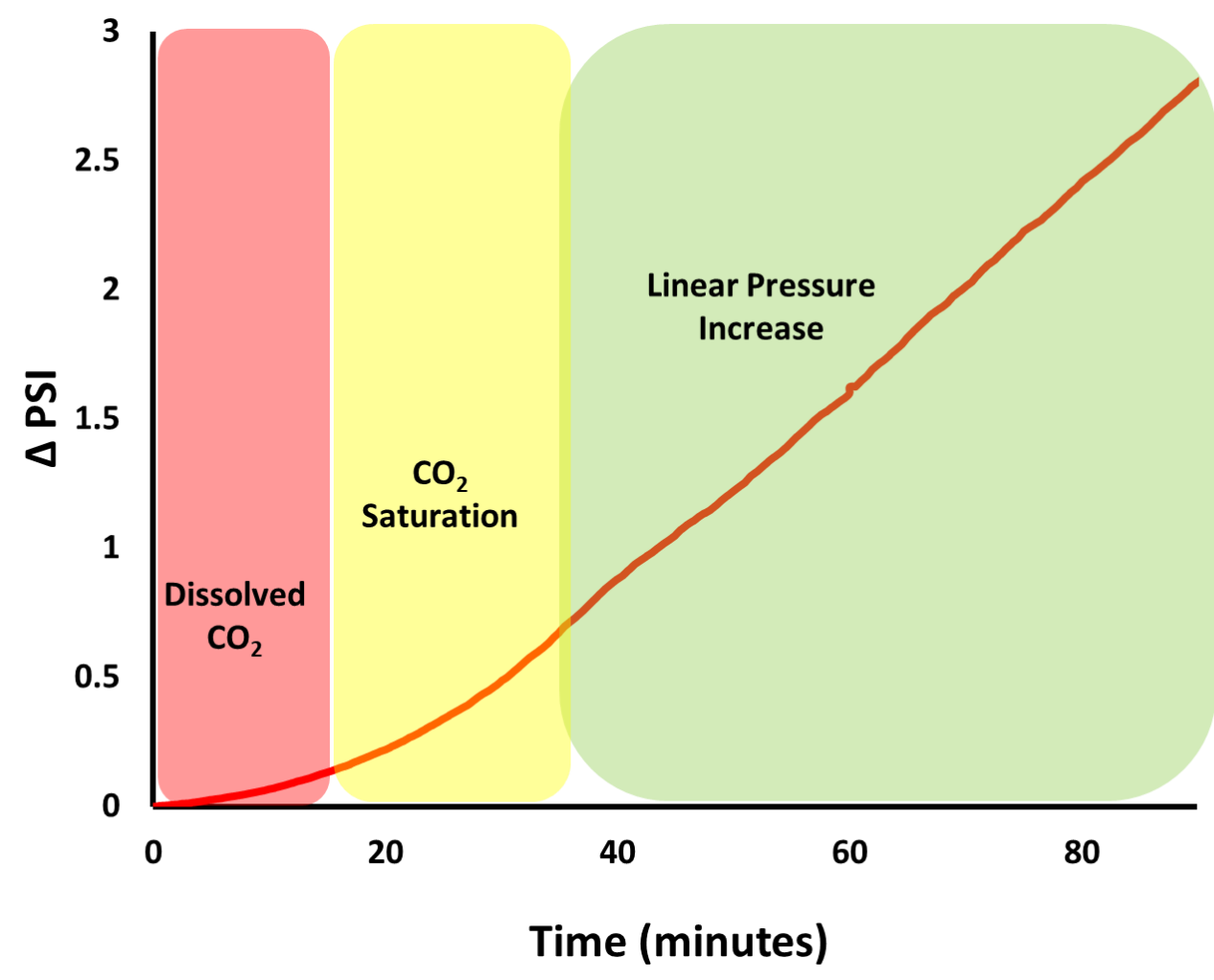

Figure 4.2. Change in pressure over time from irradiation of potassium ferrioxalate $(0.15$ M, $10 \mathrm{~mL}$ ) with blue LEDs for first 90 minutes.

Upon initial irradiation, the pressure does not increase due to the high solubility of $\mathrm{CO}_{2} . \mathrm{CO}_{2}$ has a high solubility in water and an equilibrium with carbonic acid formed. For comparison, the Henry's law constant of $\mathrm{CO}_{2}$ is $29.41 \mathrm{~atm} \mathrm{~mol}^{-1}$ as compared to $\mathrm{N}_{2}$ which has a constant of 1639 atm $\mathrm{mol}^{-1}$. The initial rate of $\mathrm{CO}_{2}$ in solution cannot be calculated because Henry's law can only be used to determine the maximum amount of gas that can be dissolved. Therefore, during the initial time period, where there is no 
pressure change, the produced $\mathrm{CO}_{2}$ remains largely dissolved in solution. Around 20 minutes, the pressure increase is a result of the $\mathrm{CO}_{2}$ saturation which appears non-linear, however, after 30 minutes a linear rate of $\mathrm{CO}_{2}$ was observed. The linear or pseudo-zeroth order rate of $\mathrm{CO}_{2}$ is expected as the reaction is only limited by the amount of photons absorbed. Furthermore, as compared to the traditional spectroscopic method of Fe(II) production, a linear increase in $\mathrm{CO}_{2}$ was also observed.

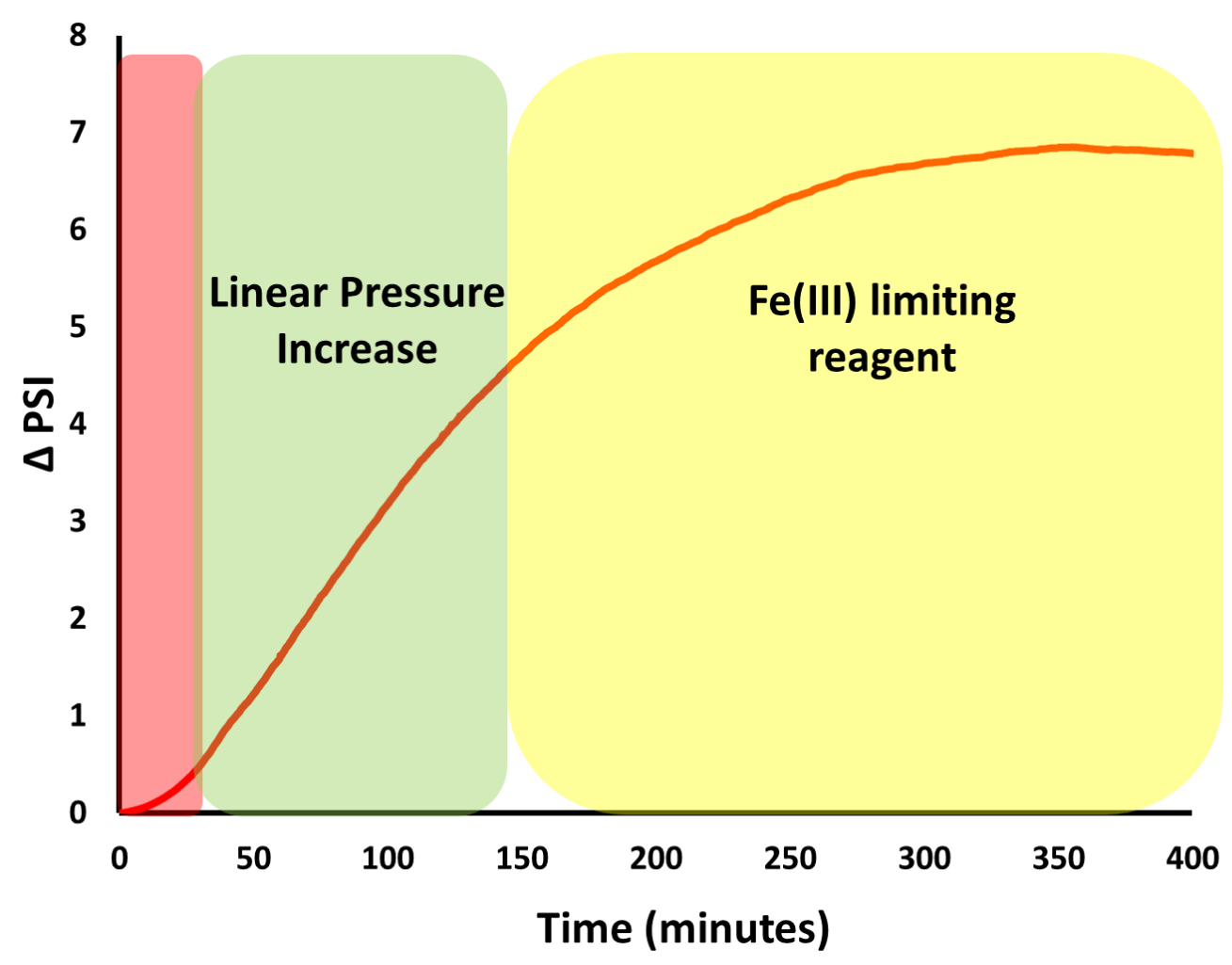

Figure 4.3. Change in pressure over time from the photoreduction of potassium ferrioxalate $(0.15 \mathrm{M}, 10 \mathrm{~mL})$ with blue LED irradiation over the course of 7 hours.

The reaction time was extended from 1 hour to 7 hours, in the resulting pressure increase with irradiation is shown in Figure 4.3. This resulted in a longer dynamic range of linear $\mathrm{CO}_{2}$ production which can be used for calculating the amount of photons 
absorbed using the rate of $\mathrm{CO}_{2}$ production. However, after around 150 minutes of irradiation there is a significant decrease in the production of $\mathrm{CO}_{2}$ as observed as a slower increase in pressure. This region is attributed to the consumption of potassium ferrioxalate which results in a slower rate of photoreduction where the absorption of light is no longer limiting the reaction. Measurements of $\mathrm{CO}_{2}$ produced from these time portions would result in erroneously low calculations of photons absorbed. The linear region of pressure increase, however, can be used to calculate the rate of photons absorbed. The determination of the amount of photons absorbed at a given time point may be calculated using equation 4.4 .

Equation 4.4. Calculation of number of photons absorbed by $\mathrm{CO}_{2}$ production

$$
n_{\text {photons }}=\frac{\text { moles of } \mathrm{CO}_{2}}{\phi_{\lambda}}
$$

Where, $n_{\text {photons }}$ is the number of photons absorbed in moles, $\phi_{\lambda}$ is the quantum yield of the reaction at the specific wavelength, in this case 0.94, and moles of $\mathrm{CO}_{2}$ is determined using Equation 4.3 at a specific time point.

The rate of photons absorbed may be calculated in a similar fashion, to determine the amount of photons absorbed per second. Utilizing the rate of $\mathrm{CO}_{2}$ produced overtime is advantageous because the slope of $\mathrm{CO}_{2}$ production over time is calculated based on hundreds of data points rather than picking a singular time point. However, it is important to note that the rate of $\mathrm{CO}_{2}$ production should only be calculated within the dynamic range of $\mathrm{CO}_{2}$ production, which in this case was found to be between 30 and 150 minutes. The rate of photons per second may then be calculated using Equation 4.5. 
Equation 4.5. Calculation of photons per second by $\mathrm{CO}_{2}$ production.

$$
\frac{n_{\text {photons }}}{\text { second }}=\frac{\frac{\text { moles of } \mathrm{CO}_{2}}{\text { seconds }}}{\phi_{\lambda}}
$$

To calculate the moles of $\mathrm{CO}_{2}$ produced over time in a closed system it is important to incorporate Henry's law at each time point. As the pressure increases the amount of $\mathrm{CO}_{2}$ that is dissolved in solution also increases. However, utilizing a change in volume instead can keep the pressure constant and the $\mathrm{CO}_{2}$ may be calculated using Equation 4.3.

\subsubsection{Calculation of photons absorbed by $\mathrm{CO}_{2}$ capture}

To avoid the need for pressure sensors an alternative method was developed in which the $\mathrm{CO}_{2}$ was captured using standard laboratory equipment. As shown in Figure 4.4, a rubber tube was used to connect the reaction vessel to a submerged graduated cylinder filled with water. Upon the production of $\mathrm{CO}_{2}$, the water can be displaced and the volume of $\mathrm{CO}_{2}$ can be used rather than the pressure increase. 

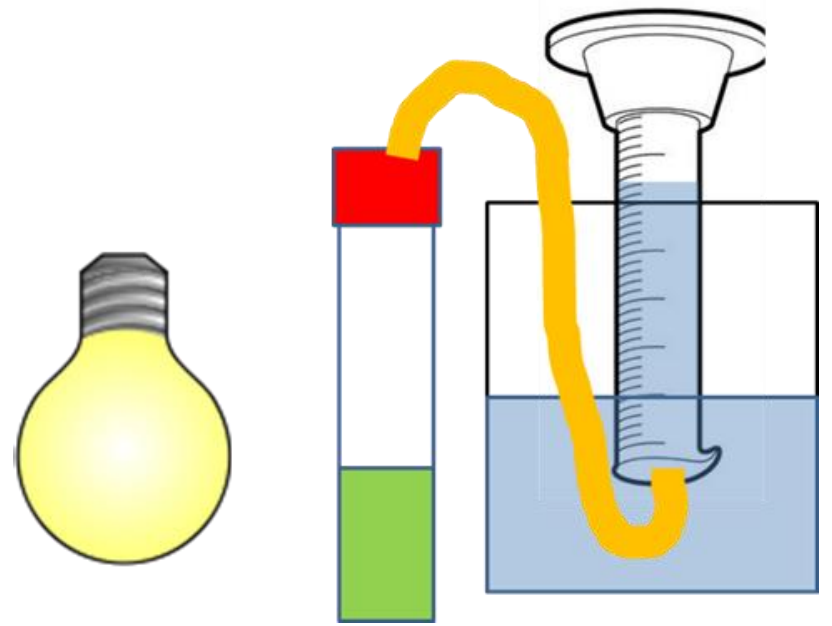

Figure 4.4. Depiction of $\mathrm{CO}_{2}$ capture method by water displacement.

Using the change in volume, to follow the photoreduction of potassium

ferrioxalate, the equation to calculate the amount $\mathrm{CO}_{2}$ produced the same as Equation 4.3, except the pressure is considered constant while volume $\left(\mathrm{V}_{1}\right)$ changes instead. Therefore, the addition of Henry's law constant, to determine the amount of $\mathrm{CO}_{2}$ dissolved, stays constant because the pressure is assumed constant.

The change in volume over time showed similar trends to monitoring the change in pressure over time. The initial period up to 20 minutes was not used because of the dissolution of $\mathrm{CO}_{2}$ in solution. However, the measured amount of $\mathrm{CO}_{2}$ at 40 and 60 minutes was in good agreement with the prior two methods. The results from each three methods (Fe(II) production, change in pressure, change in volume) can be seen in Figure 4.5 . 


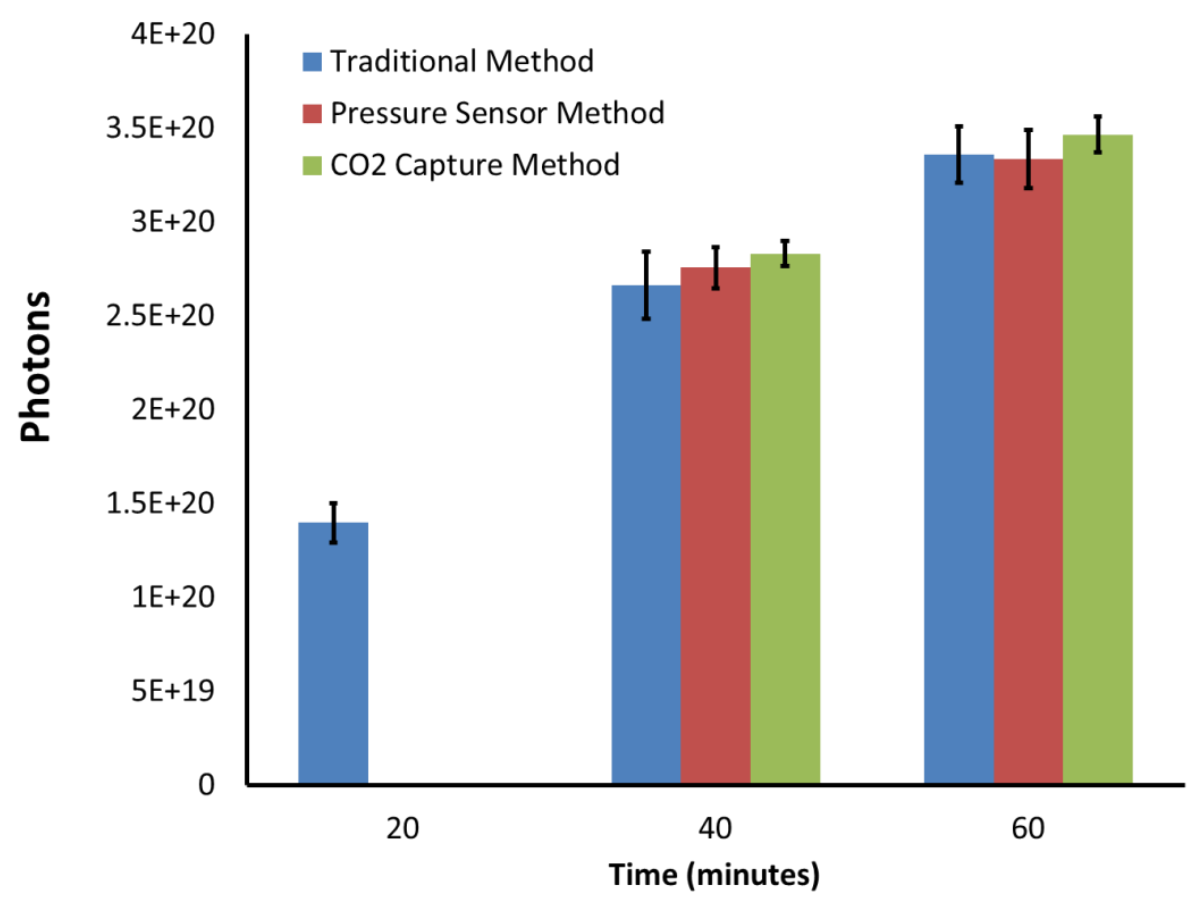

Figure 4.5. Calculated amount of photons absorbed by the three methods.

\subsection{Conclusions}

Chemical actinometry is a necessary photochemical measurement to determine the quantum yield of a light driven reaction. The photoreduction of potassium ferrioxalate is one of the most common chemical actinometers used. The amount of solution preparation, transfers, and waiting periods make this method tedious and leave little room for error. For these reasons, quantum yield measurements typically have an assumed $10 \%$ margin of error. We have shown two alternative approaches to this method which rely on measuring the amount of $\mathrm{CO}_{2}$ produced by physical methods rather than the amount of $\mathrm{Fe}(\mathrm{II})$ which is typically measured spectroscopically. Measuring $\mathrm{CO}_{2}$ comes 
with its own advantages and disadvantages compared to the traditional spectroscopic method.

In both $\mathrm{CO}_{2}$ measurements, by volume or by pressure, at early time periods (prior to 30 minutes of irradiation) it was difficult to calculate the amount of $\mathrm{CO}_{2}$ produced. This is a result of the high solubility of $\mathrm{CO}_{2}$ in water. Further research may focus on electrolytes or other compounds to lower the solubility of the $\mathrm{CO}_{2}$ to avoid such shortcomings. However, it should be considered that the effect of additional chemical species in solution may alter the quantum yield of the reaction.

The advantage of measuring $\mathrm{CO}_{2}$ at later time periods, between 30 and 150 minutes, is the decreased chance for incident light exposure. The samples are not moved during the course of the measurement and the measurement is collected in real-time. Furthermore, the vast number of data points that can be collected from a single sample is far superior to the $\mathrm{Fe}(\mathrm{II})$ detection method which yields only one data point per sample. There is no dissolution or waiting periods in the dark which is also beneficial for adopting this methodology for widespread implementation.

\subsection{Materials and Methods}

\subsubsection{Synthesis of potassium ferrioxalate}

Potassium ferrioxalate, $\mathrm{K}_{3} \mathrm{Fe}\left(\mathrm{C}_{2} \mathrm{O}_{4}\right)_{3} \bullet 3 \mathrm{H}_{2} \mathrm{O}$, was prepared by mixing aqueous solutions of $\mathrm{K}_{2} \mathrm{C}_{2} \mathrm{O}_{4}(1.5 \mathrm{M}, 300 \mathrm{~mL})$ with $\mathrm{K}_{3} \mathrm{Fe}\left(\mathrm{C}_{2} \mathrm{O}_{4}\right)_{3} \bullet 3 \mathrm{H}_{2} \mathrm{O}$ (1.5 M, $\left.100 \mathrm{~mL}\right)$. Upon mixing the two solutions an immediate change from colorless to green occurred with 
green crystals precipitating. The solution was left for 10 minutes without stirring and then vacuum filtered and dried at $60{ }^{\circ} \mathrm{C}$ overnight.

\subsubsection{Traditional method determining $\mathrm{Fe}(\mathrm{II})$ concentration}

All solution preparation took place in a novel light free set up with the exception of a red LED light for safety (Figure 4.6). The samples were prepared by adding $\sim 0.75 \mathrm{~g}$ of $\mathrm{K}_{3} \mathrm{Fe}\left(\mathrm{C}_{2} \mathrm{O}_{4}\right)_{3} \bullet 3 \mathrm{H}_{2} \mathrm{O}$, to $1 \mathrm{~mL}$ of $1.0 \mathrm{M} \mathrm{H}_{2} \mathrm{SO}_{4}$, and $9 \mathrm{~mL}$ of $\mathrm{DI} \mathrm{H}_{2} \mathrm{O}$ to produce a $0.15 \mathrm{M}$ solution in triplicate samples. The solutions were then transferred in covered containers to the light source and were irradiated with blue LEDs while stirring at $500 \mathrm{rpm}$. At times 20, 40, and 60 minutes a $0.1 \mathrm{~mL}$ aliquot was taken from each sample and added to a 100 $\mathrm{mL}$ volumetric flask. The aliquot was then diluted using $5 \mathrm{~mL}$ of an aqueous 1,10 phenantthroline solution ( $0.1 \%$ by weight), $1 \mathrm{~mL}$ of a sodium acetate buffer, and diluted with DI water. The solutions were shaken and allowed to sit in the dark for one hour before the absorbance at $511 \mathrm{~nm}$ was measured by UV-Vis absorption spectroscopy. 


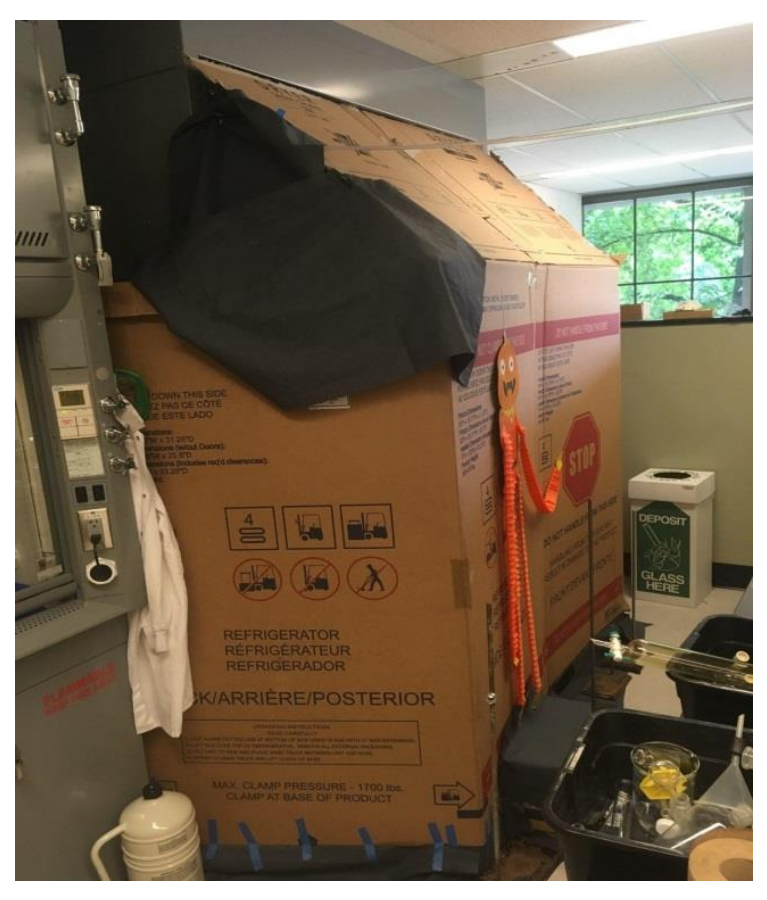

Figure 4.6. Picture of dark room set up for solution preparation.

\subsubsection{Determination of carbon dioxide by pressure change}

Potassium ferrioxalate solutions $(0.15 \mathrm{M}, 10 \mathrm{~mL})$ were prepared identically to the traditional method and transported to LEDs in a covered container. The solutions were capped using pressure sensors and the pressure was measured every 30 seconds for 7 hours total.

\subsubsection{Determination of carbon dioxide by volume change method}

Solutions of potassium ferrioxalate $(0.15 \mathrm{M}, 10 \mathrm{~mL})$ were put in the same reaction vessel as for the pressure change and traditional method but capped with a septum. The septum was pierced with a syringe connected to a hose fed to an inverted submerged graduated cylinder (Figure 4.4). Before irradiation, a syringe full of air was injected through the septum to evacuate any water in the hose. The solution was then irradiated 
with blue LEDs for 90 minutes and the change in volume in the graduated cylinder was

recorded every ten minutes.

\subsection{References}

1. Lang, X., Chen, X. \& Zhao, J. Heterogeneous visible light photocatalysis for selective organic transformations. Chem. Soc. Rev. 43, 473-486 (2014).

2. Ravelli, D., Fagnoni, M. \& Albini, A. Photoorganocatalysis. What for? Chem. Soc. Rev. 42, 97-113 (2013).

3. Prier, C. K., Rankic, D. A. \& MacMillan, D. W. C. Complexes : Applications in Organic Synthesis. Chem Rev. 113, 5322-5363 (2013).

4. Roibu, A. et al. An accessible visible-light actinometer for the determination of photon flux and optical pathlength in flow photo microreactors. Sci. Rep. (2018) doi:10.1038/s41598-018-23735-2.

5. Asenath-Smith, E., Ambrogi, E. K., Moores, L. C., Newman, S. D. \& Brame, J. A. Leveraging chemical actinometry and optical radiometry to reduce uncertainty in photochemical research. J. Photochem. Photobiol. A Chem. (2019) doi:10.1016/j.jphotochem.2018.12.024.

6. Youngblood, W. J., Lee, S.-H. A., Maeda, K. \& Mallouk, T. E. ChemInform Abstract: Visible Light Water Splitting Using Dye-Sensitized Oxide Semiconductors. ChemInform 41, (2010).

7. Barber, J. Photosynthetic energy conversion: Natural and artificial. Chem. Soc. Rev. 38, 185-196 (2009).

8. Berardi, S. et al. Molecular artificial photosynthesis. Chem. Soc. Rev. 43, 75017519 (2014).

9. Rubin, M. B. \& Braslavsky, S. E. Quantum yield: The term and the symbol. A historical search. Photochem. Photobiol. Sci. 9, 670-674 (2010).

10. Maschmeyer, T. \& Che, M. Catalytic aspects of light-induced hydrogen generation in water with $\mathrm{TiO} 2$ and other photocatalysts: a simple and practical way towards a normalization? Angew. Chemie - Int. Ed. 49, 1536-1539 (2010).

11. Demas, J. N., Bowman, W. D., Zalewski, E. F. \& Velapoldi, R. A. Determination of the quantum yield of the ferrioxalate actinometer with electrically calibrated radiometers. J. Phys. Chem. (1981) doi:10.1021/j150619a015. 
12. Receive, F. A new sensitive chemical actinometer. I. Some trials with potassium ferrioxalate. Proc. R. Soc. London. Ser. A. Math. Phys. Sci. 220, 104-116 (2006).

13. Hatchard, C. G. \& Parker, C. A. A New Sensitive Chemical Actinometer. II. Potassium Ferrioxalate as a Standard Chemical Actinometer. Proc. R. Soc. A Math. Phys. Eng. Sci. (1956) doi:10.1098/rspa.1956.0102.

14. Kuhn, H. J., Braslavsky, S. E. \& Schmidt, R. Chemical actinometry (IUPAC Technical Report). Pure Appl. Chem. 76, 2105-2146 (2007).

15. Rabek, J. F. Experimental Methods in Photochemistry and Photophysics. (John Wiley \& Sons, 1982).

16. Ji, Y., DiRocco, D. A., Hong, C. M., Wismer, M. K. \& Reibarkh, M. Facile Quantum Yield Determination via NMR Actinometry. Org. Lett. (2018) doi:10.1021/acs.orglett.8b00391. 


\section{Predictive Modeling of Heavy Atom Effects in Halogenated Fluorescein Derivatives}

\subsection{Background}

The development of chromophores with significant rates of ISC to produce excited triplet-states (high $\phi_{\mathrm{t}}$ ) is important for the development of photocatalysts, LEDs, and ${ }^{1} \mathrm{O}_{2}$ sensitizers. ${ }^{1-3}$ Designing chromophores with high $\phi_{\mathrm{t}}$ is often difficult as minor structural modification of existing chromophores can result in significant changes to the $\phi_{\mathrm{t}}$. As such, the $\phi_{\mathrm{t}}$ of a chromophore is often explained post synthesis and characterization. ${ }^{4-6}$ Determining the structural properties that result in high $\phi_{\mathrm{t}}$ is key to developing predictive models for the rational design of triplet photosensitizers. Such predictive power can alleviate synthetic labor required to synthesize and characterize chromophore derivatives and novel compounds.

One technique to increase the $\phi_{\mathrm{t}}$ of a chromophore is the addition of heavy atoms. The heavy atom effect is used to describe the increased spin orbit coupling observed in compounds containing atoms with high atomic number $(\mathrm{Z})$. The increased $\mathrm{Z}$ results in an increased nuclear charge and therefore magnetic moment. Electrons within the viscinity of the heavy atom can be influenced through spin coupled interactions with the orbital from this magnetic moment, thus increasing the rate of ISC. ${ }^{1}$ Traditionally, the strength of the spin orbit coupling interaction has only been predicted from a monoatomic point of view. Recently, it has been shown in selenium containing chromophores that the 
computationally determined contribution of the heavy atom (selenium) to the LUMO resulted in an increased $\phi_{\Delta}$ which was used as a lower bound approximation for the $\phi_{\mathrm{t} .}{ }^{6}$ Four different chromophores with selenium in different positions were synthesized, characterized and computationally modeled (Figure 5.1). It was found that the position of the selenium atom was directly related to its LUMO contribution and to the $\phi_{\Delta .}$

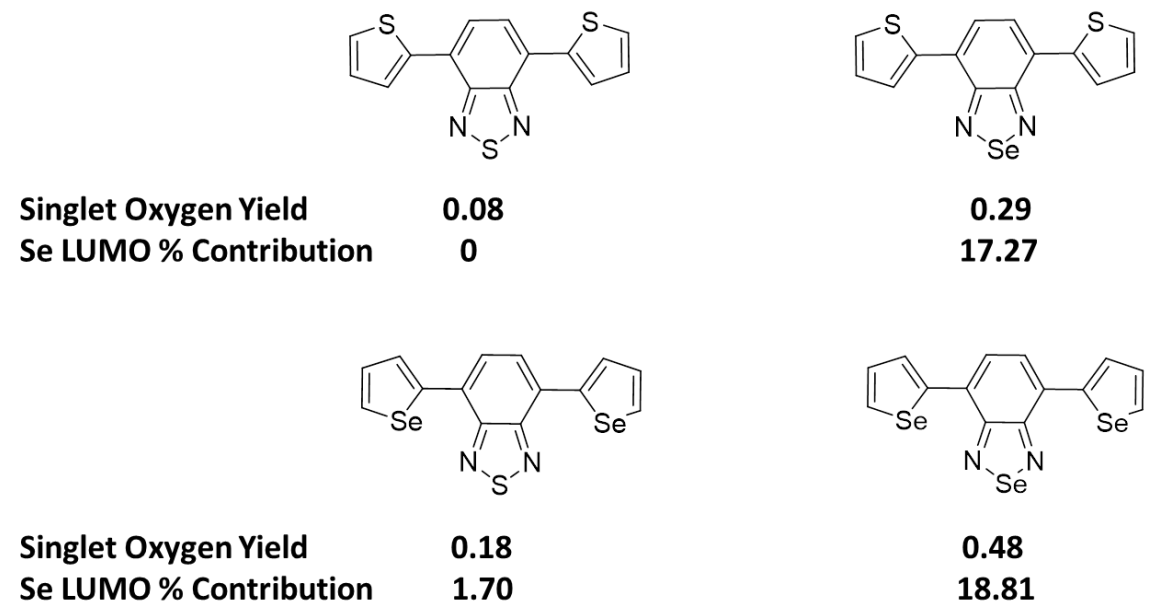

Figure 5.1. Heavy atom contribution to the LUMO and triplet yield from selenium containing chromophores. ${ }^{6}$

Herein we use a similar approach to model the heavy atom contributions from various halogenated fluorescein derivatives with known $\phi_{\Delta}$. Chromophores containing the heavy atoms bromine and iodine were used. It was found that the $\phi_{\Delta}$ and heavy atom contribution to the triplet-state LUMO was dependent on the identity and position of the heavy atoms. From this, a predictive model was developed. To test the predictive power of the model, two fluorescein derivatives with unreported singlet oxygen yields were synthesized and their experimental $\phi_{\Delta}$ and predicted $\phi_{\Delta}$ were compared. 


\subsection{Results and Discussion}

\subsubsection{Computational modeling of halogenated fluorescein derivatives}

Scheme 5.1. General halogenation pattern of fluorescein derivatives.<smiles>[R]c1cc2c(-c3c([R])c([R])c([R])c([R])c3[R])c3cc([R2])c(=O)c([R])c-3oc2c([R3])c1[O-]</smiles>

Halogenation of fluorescein dyes characteristically show a red-shifted absorbance as compared to fluorescein. Iodination or bromination of fluorescein typically results in higher singlet oxygen quantum yields and decreased fluorescence quantum yields as compared to chlorinated or unhalogenated fluorescein. ${ }^{7}$ The increased rates of ISC result in a higher triplet yield which in turn can be approximated by the singlet oxygen yield of the dyes. However, it has been previously shown that the position is of great importance to how much the singlet oxygen quantum yield is increased from the parent compound. The present study aimed to determine if the contribution of the heavy atoms to the frontier orbitals could be quantified and used as a predictive model to calculate the singlet oxygen quantum yield of other derivatives. Computational chemistry was used to model seven different fluorescein derivatives containing bromine or iodine as heavy atoms in different positions. 
Table 5.1. Fluorescein derivatives that were computationally modeled and their reported singlet oxygen quantum yields in $\mathrm{H}_{2} \mathrm{O}$.

\begin{tabular}{lllll}
\hline \multicolumn{1}{c}{ Fluorescein Derivative } & $\mathbf{R}_{\mathbf{1}}$ & $\mathbf{R}_{\mathbf{2}}$ & $\mathbf{R}_{\mathbf{3}}$ & $\boldsymbol{\varphi}_{\mathbf{\Delta}}{ }^{8}$ \\
\hline Rose Bengal & $\mathrm{Cl}$ & $\mathrm{I}$ & $\mathrm{I}$ & 0.76 \\
Phloxin B & $\mathrm{Cl}$ & $\mathrm{Br}$ & $\mathrm{Br}$ & 0.65 \\
Eosin Y & $\mathrm{H}$ & $\mathrm{Br}$ & $\mathrm{Br}$ & 0.57 \\
Erthrosine & $\mathrm{H}$ & $\mathrm{I}$ & $\mathrm{I}$ & 0.68 \\
3',4',5',6'-tetrabromo & $\mathrm{Br}$ & $\mathrm{H}$ & $\mathrm{H}$ & 0.20 \\
4,5-dibromo & $\mathrm{H}$ & $\mathrm{H}$ & $\mathrm{Br}$ & 0.42 \\
4,5-diiodo & $\mathrm{H}$ & $\mathrm{H}$ & $\mathrm{I}$ & 0.48 \\
\hline
\end{tabular}

While a wide variety of halogenated derivatives have been reported, the seven that were chosen to model are of the most well studied. Often there are large discrepancies in the literature for singlet oxygen quantum yields for the same compounds even under the same conditions. Derivatives with a wide variance in reported singlet oxygen quantum yields were not included because potential influence on the model from choosing the incorrect reported value. The seven different known compounds were optimized in the triplet state using Density Functional Theory (DFT) at a B3LYP level of theory. A split basis set was used treating $\mathrm{C}, \mathrm{H}, \mathrm{O}, \mathrm{Cl}$, and $\mathrm{Br}$ atoms with 6-311 $\mathrm{G}(\mathrm{d})$ and LANL2dz for I atoms. ${ }^{9}$ Heavy atom contributions were determined using the natural atomic orbital method using Multiwfn. The resulting heavy atom contributions from $\mathrm{Br}$ or I were then plotted against the literature singlet oxygen yield of the respective derivatives as seen in Figure 5.2. 


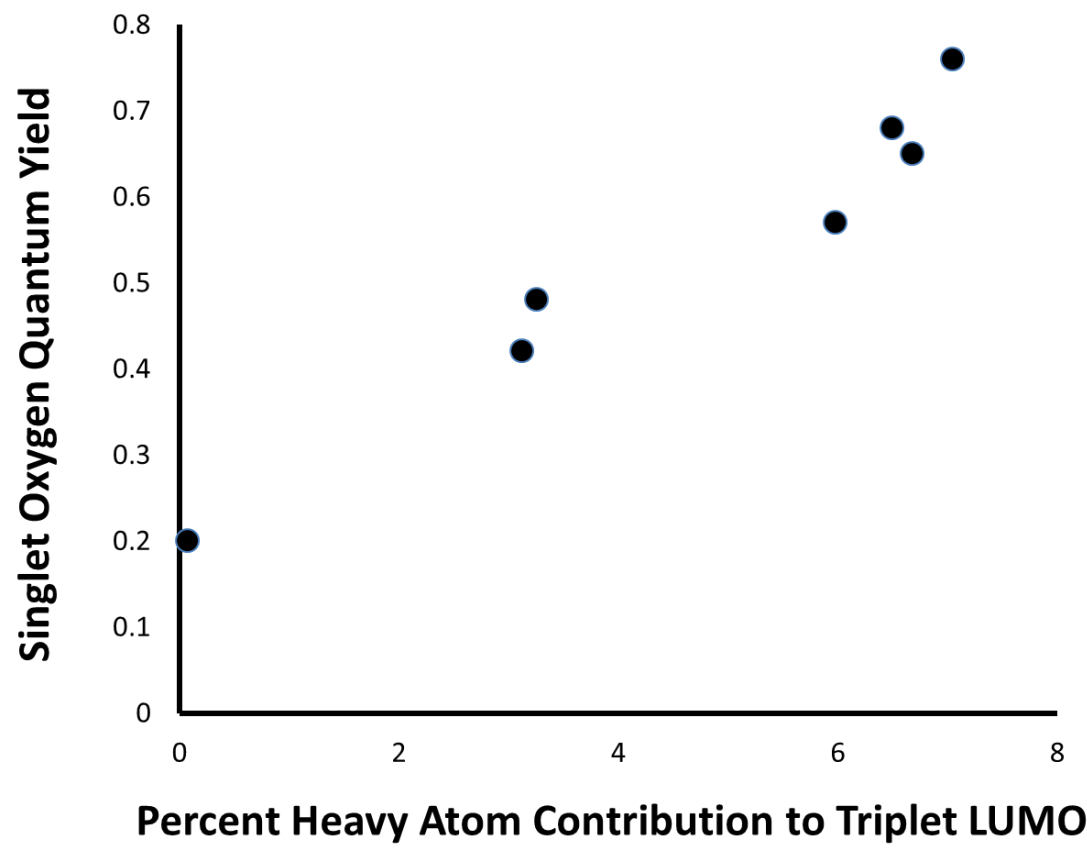

Figure 5.2. Computationally determined heavy atom contribution to the triplet LUMO compared to the literature singlet oxygen quantum yield.

The resulting plot shows a progressive increase of singlet oxygen yield compared to the heavy atom contribution. However, the model does not take into account the heavy atoms identity, $\mathrm{Br}$ or I. The rate of ISC is known to increase proportionally with the atomic number $(Z)$ of the heavy atom. As a rudimentary method to incorporate this trend, the heavy atom contribution from I or Br was multiplied by the halogen atomic number, 35 or 53 . The following equation was then used to determine the adjusted heavy atom contribution compared to the reported singlet oxygen yield. 
Equation 5.1. Calculation of adjusted heavy atom contribution.

Adjusted Heavy Atom Contrubtion $=\sum\left(\frac{\% \text { contrıutıon }}{100} \times Z_{\text {Halogen }}\right)$

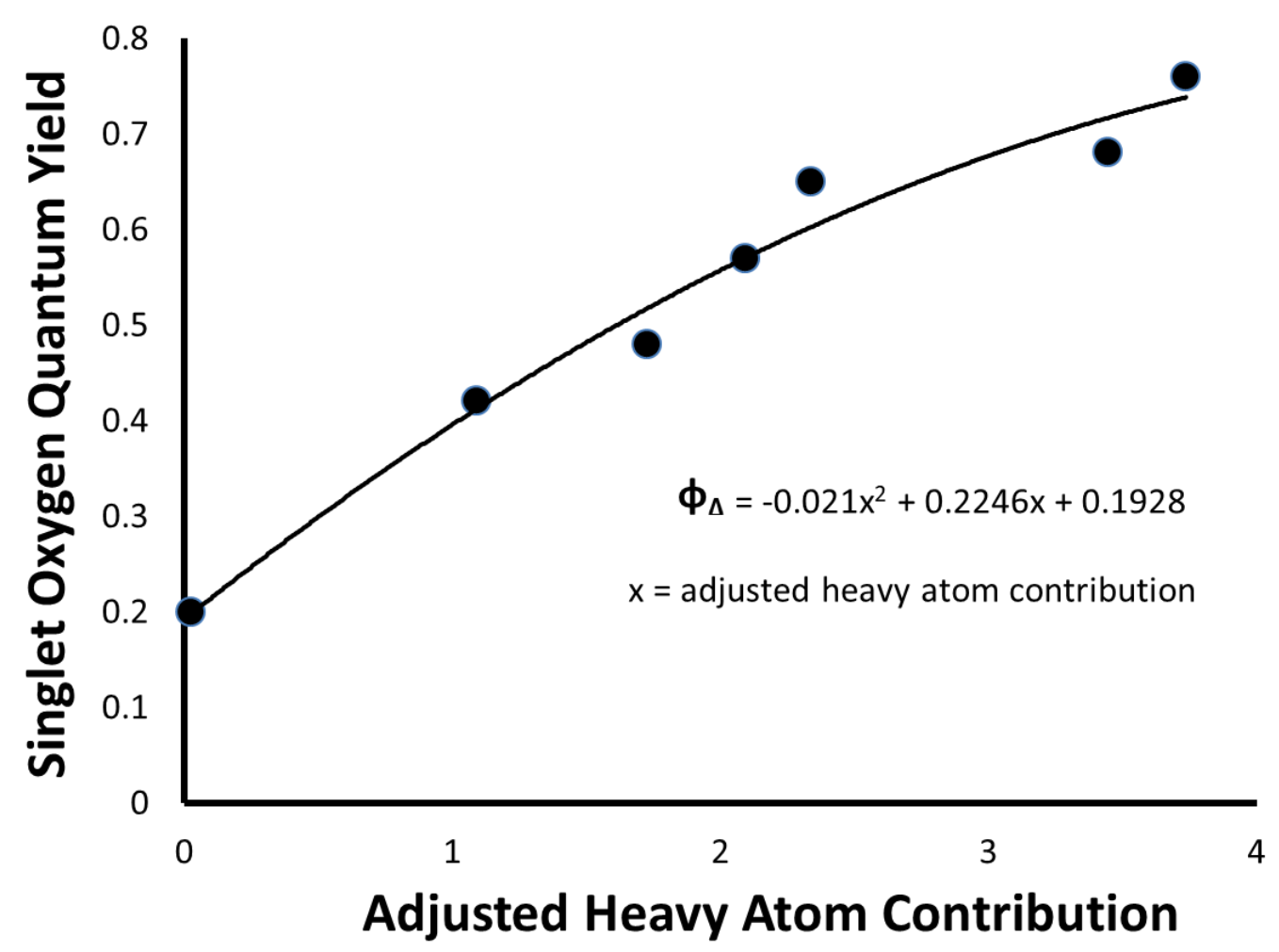

Figure 5.3. Plot of the adjusted computationally determined heavy atom contribution compared to the literature reported singlet oxygen quantum yield.

The resulting plot shows that the increase in heavy atom contribution results in an increase in singlet oxygen yield. The model makes some key distinctions between the position and the identity of the heavy atoms. For example, 3', 4', ,', '6'-tetrabromo fluorescein may be expected to have a higher singlet oxygen yield than 4,5-dibromo if 
only the number of heavy atoms were considered. However, the contribution of the halogens from the top ring $\left(4^{\prime}-6^{\prime}, \mathrm{R}_{1}\right)$ is negligible compared to the lower 4 and $5\left(\mathrm{R}_{3}\right)$ positions. The calculations show that 4,5-dibromo fluorescein has a higher adjusted heavy atom contribution which is seen experimentally in the higher singlet oxygen yield. Furthermore, when comparing 4,5-dibromo and 4,5-diiodo fluorescein, adjusting the contribution by multiplying by $Z$, 4,5-diiodo has a significantly higher heavy atom contribution which is observed with a significantly higher singlet oxygen yield. Fitting a second order polynomial to the data allows the $\phi_{\Delta}$ to be predicted by the calculated adjusted heavy atom contribution. A second order fit was chosen to limit the calculated $\phi_{\Delta}$ to below the maximum possible quantum yield of 1 . To test the predictive power of the model, two fluorescein derivatives were unreported singlet oxygen yields were synthesized and the photophysical properties were examined.

\begin{tabular}{lccc}
\hline \multicolumn{1}{c}{$\begin{array}{c}\text { Fluorescein } \\
\text { Derivative }\end{array}$} & $\begin{array}{c}\text { \% Heavy atom } \\
\text { contribution }\end{array}$ & $\begin{array}{c}\text { Adjusted heavy atom } \\
\text { contribution }\end{array}$ & $\boldsymbol{\varphi}_{\Delta}{ }^{8}$ \\
\hline Rose Bengal & 7.05 & 3.73 & 0.76 \\
Phloxin B & 6.68 & 3.45 & 0.65 \\
Eosin Y & 5.98 & 2.09 & 0.57 \\
Erthrosine & 6.50 & 3.45 & 0.68 \\
3',4',5',6'-tetrabromo & 0.07 & 0.02 & 0.20 \\
4,5-dibromo & 3.12 & 1.09 & 0.42 \\
4,5-diiodo & 3.26 & 1.72 & 0.48 \\
\hline
\end{tabular}

Table 5.2. Computationally determined heavy atom contributions from known fluorescein derivatives.

\subsubsection{Synthesis of unreported fluorescein derivatives}


The synthesis of two previously reported fluorescein derivatives was undertaken to test the predictive power of the computational model created. Both dyes were synthesized by the solid state reaction of tetrahalogenated phthalic anhydride with resorcinol and catalyzed using zinc chloride (Scheme 5.2). Further halogenation was achieved using post synthetic methods to add bromine or iodine to the respective dyes.

Scheme 5.2. Synthesis of unreported halogenated derivatives<smiles>[X]c1c([X])c([X])c2c(c1[X])C(=O)OC2=O</smiles><smiles>O=C(O)c1c(Br)c(Br)c(Br)c(Br)c1-c1c2ccc(=O)cc-2oc2cc(O)ccc12</smiles><smiles>[GeH3]</smiles><smiles></smiles>

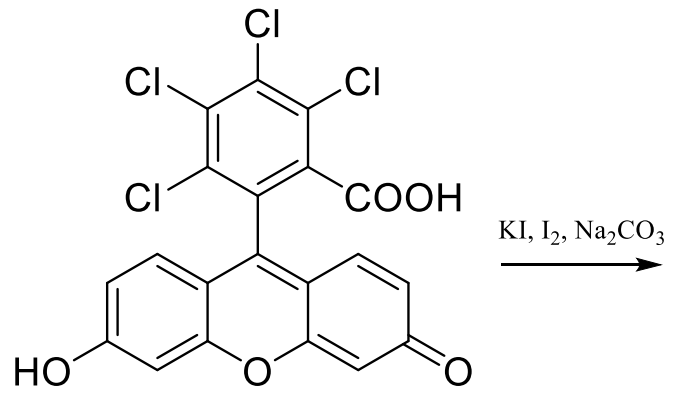<smiles>O=C(O)c1c(Cl)c(Cl)c(Cl)c(Cl)c1-c1c2ccc(=O)c(I)c-2oc2c(I)c(O)ccc12</smiles> 
5.2.2. Photophysical properties of halogenated derivatives

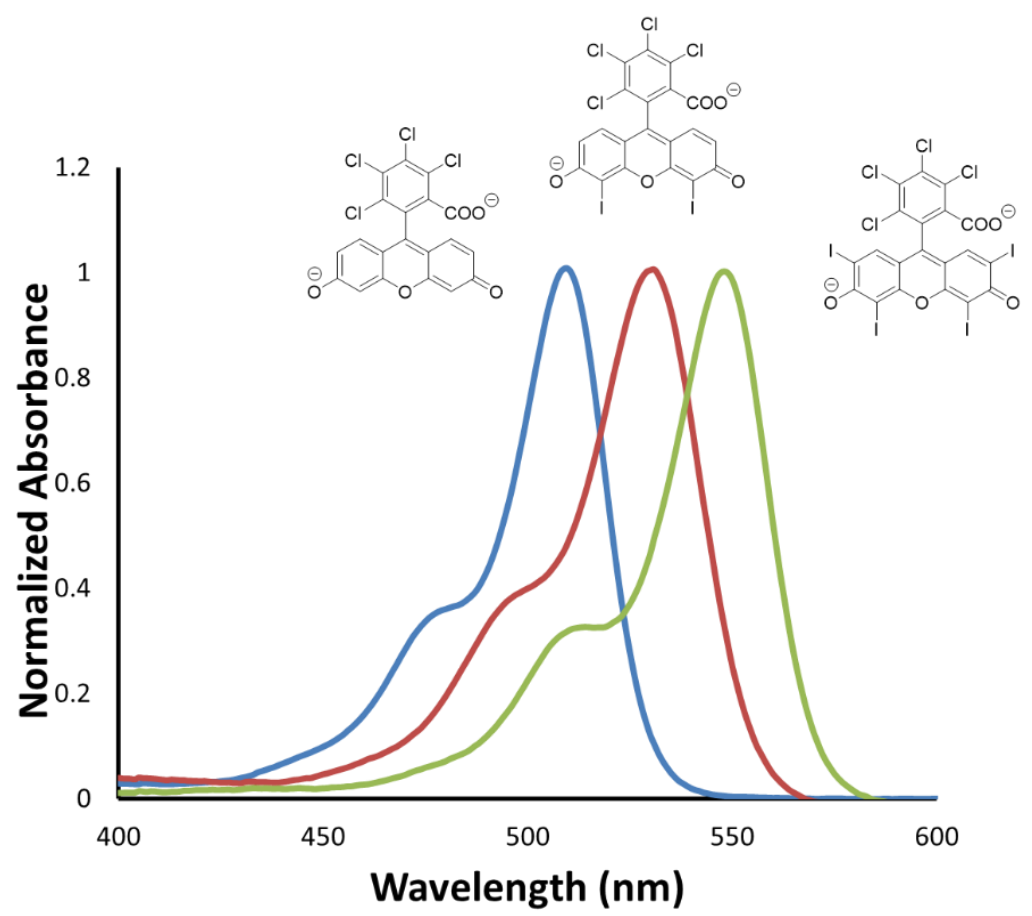

Figure 5.4. UV-Vis absorbance spectra of prepared dyes compared to Rose Bengal for reference.

The synthesized dyes, octabromo fluorescein $(\mathrm{FBr})$ and 3', 4',5',6'-tetrachlor-4,5diiodo fluorescein (FI), were found to have red shifted absorbance compared to unhalogenated fluorescein. The absorbance of FI has an absorbance max at $530 \mathrm{~nm}$ which places it between 3',4',5',6'-tetrachloro fluorescein and Rose Bengal. The absorbance of FBr shows a similar bathochromic shift as compared to its starting material of $3^{\prime}, 4^{\prime}, 5^{\prime}, 6^{\prime}-$ tetrabromo fluorescein, Figure 5.4. 


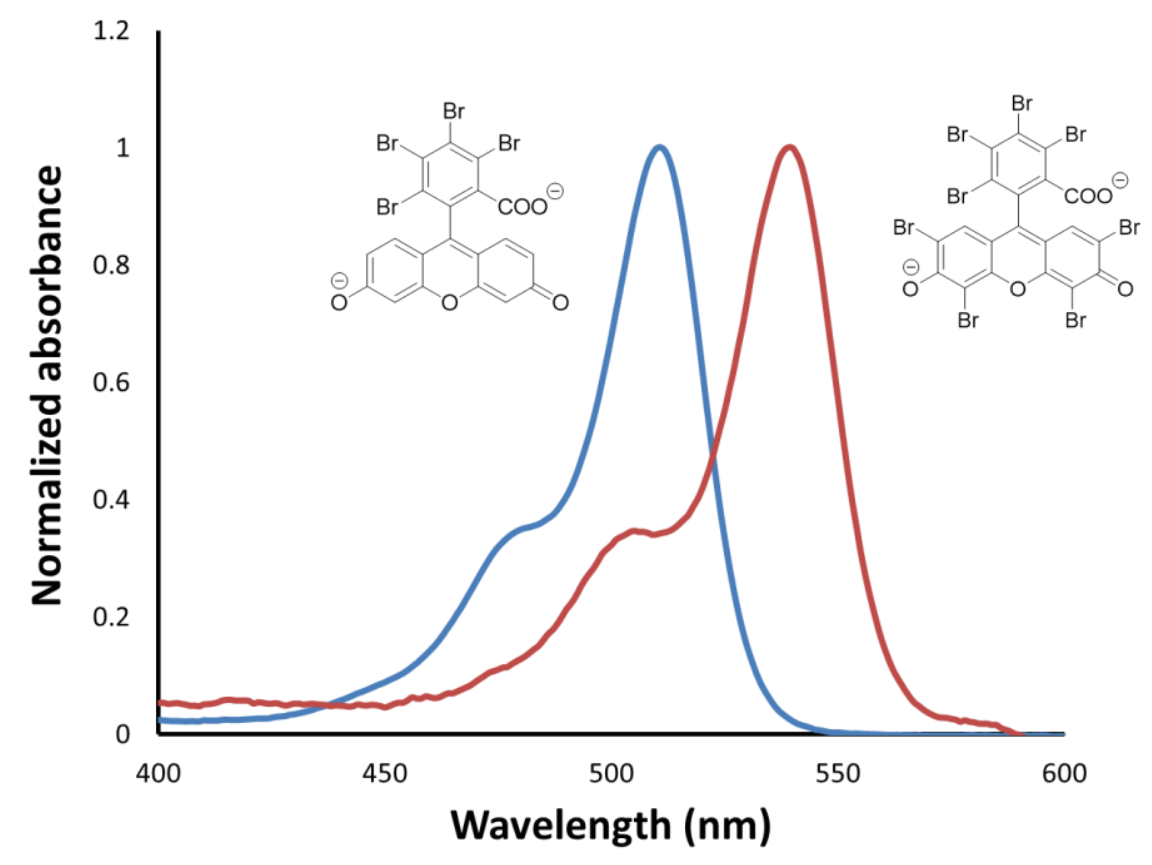

Figure 5.4. UV-Vis absorbance of FBr8 compared to tetrabromo fluorescein for reference.

Both synthesized derivatives were found to be visibly luminescent under black light $(365 \mathrm{~nm})$ irradiation. Fluorescence spectra of the compounds were compared to Rose Bengal as a standard which has a known fluorescence quantum yield $\left(\phi_{f}\right)$ of 0.016 .

FI displayed a similar $\phi_{\mathrm{f}}$ to that of Rose Bengal around 0.01 despite having a lower anticipated $\phi_{\Delta} . \mathrm{FBr}$, interestingly, was an order of magnitude more luminescent with a $\phi_{\mathrm{f}}$ of 0.17. The short Stokes shift between the absorbance and emission of the compounds is characteristic of fluorescence rather than phosphorescence.

The $\phi_{\Delta}$ of FBr and FI were determined experimentally using the previously described method of oxygen consumption. FBr was found to have a $\phi_{\Delta}$ of 0.65 while FI was lower at 0.51 as compared to Rose Bengal as a standard $\left(\phi_{\Delta}=0.76\right)$. The 
experimentally determined $\phi_{\Delta}$ were in good agreement with their predicted values as discussed below.

The two fluorescein derivatives were also computationally modeled in an identical manner to the literature derivatives. $\mathrm{FBr}$ was determined to have a heavy atom contribution to the triplet LUMO of $7.05 \%$ and adjusted value of 2.46 . FI was found to have a heavy atom contribution of $3.26 \%$ and adjusted contribution of 1.73 . Based on the model developed from known fluorescein derivatives, it is expected that $\mathrm{FBr}$ should have a higher $\phi_{\Delta}$ than FI. Using the adjusted heavy atom contributions it was calculated that $\mathrm{FBr}$ and FI are expected to have $\phi_{\Delta}$ of 0.61 and 0.53 , respectively. A summary of the experimental photophysical properties and calculated $\phi_{\Delta} \mathrm{O} F \mathrm{FBr}$ and $\mathrm{FI}$ is provided in Table 5.3.

Table 5.3. Summary of photophysical properties of FI and $\mathrm{FBr}$

\begin{tabular}{|c|c|c|c|c|c|}
\hline Synthesized derivative & $\begin{array}{c}\text { Abs } \lambda_{\text {max }} \\
\text { (nm) }\end{array}$ & $\begin{array}{c}\text { Emission } \\
\lambda_{\max }(\mathrm{nm})\end{array}$ & $\varphi_{\mathrm{f}}$ & $\begin{array}{c}\varphi_{\Delta} \\
\text { Calculated }\end{array}$ & $\begin{array}{c}\varphi_{\Delta} \\
\text { Experimental }\end{array}$ \\
\hline Octabromo $(\mathrm{FBr})$ & 540 & 557 & 0.17 & 0.61 & 0.65 \\
\hline $3^{\prime}, 4^{\prime}, 5^{\prime}, 6^{\prime}$-tetrachlor-4,5-diiodo & 530 & 549 & 0.01 & 0.53 & 0.51 \\
\hline
\end{tabular}
(FI)

The results indicate that the predictive modeling of heavy atom contributions can allow for an estimate of the $\phi_{\Delta}$. The model is able to predict that the chlorinated top ring FI slightly increase the contribution from I atoms at the 4 and 5 positions compared to 
4,5-diiodo fluorescein. The increased heavy atom contribution results in a slightly higher $\phi_{\Delta}$ of FI at 0.51 experimentally or 0.53 calculated while 4,5 -diiodo fluorescein has a literature $\phi_{\Delta}$ of 0.48 . Similarly, FBr differs from Eosin Y only by the brominated top ring compared to Eosin Y's unhalogenated top ring. The model predicts that the $\phi_{\Delta}$ should increase in FBr compared to Eosin $\mathrm{Y}$ due to the increased heavy atom contribution from Br. Interestingly, this increased contribution was from the 4,5,2,7 $\left(\mathrm{R}_{2}, \mathrm{R}_{3}\right)$ positions and not due to the added bromines on $3^{\prime}, 4^{\prime}, 5^{\prime}, 6^{\prime}\left(\mathrm{R}_{1}\right)$ positions of the top ring.

\subsection{Conclusion}

The ability to predict $\phi_{\Delta}$ has significant implications in the rational design of photocatalysts. The ability to predict such photophysical properties would spare significant synthetic expenses to increase the triplet yield of a photosensitizer. Inversely, such predictive power can allow for the substitution of heavy atoms at certain positions that do not significantly contribute to the frontier orbitals which would allow for the retention of high a singlet yield and potentially a high $\phi_{\mathrm{f}}$.

The simple model presented allowed for several key predictions on the placement of bromine and iodine on fluorescein derivatives. For example, the model shows that substitution at the $3^{\prime}, 4^{\prime}, 5^{\prime}, 6^{\prime}$ positions have a low contribution which explains the low $\phi_{\Delta}$ of tetrabromofluorescein $(0.20)$. When tested, using two unreported derivatives, the model was able to predict $\phi_{\Delta}$ in good agreement with those experimentally determined. 
While the model proposed is promising using fluorescein derivatives, it is yet unproven in other types of triplet photosensitizers and their respective derivatives.

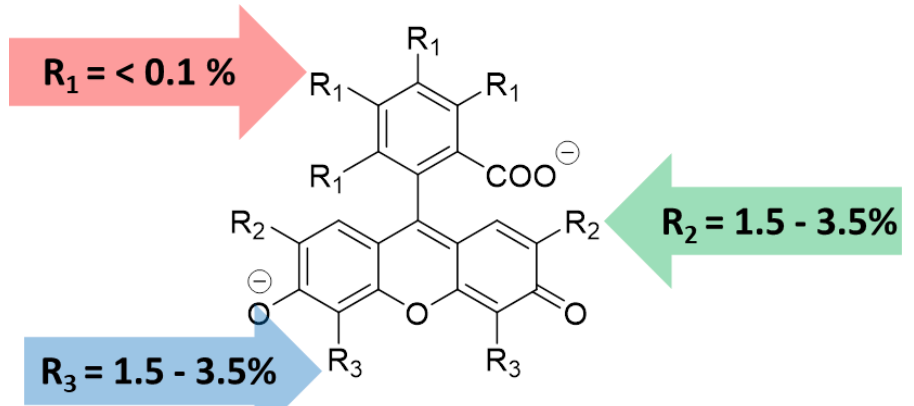

Figure 5.5. General computationally determined heavy atom contributions to the LUMO by position.

The key issue with using such a model is that it is an oversimplification of the complex relaxation pathways of a chromophore. Specifically ISC, does not solely depend on heavy atom effects. Many chromophores with high $\phi_{\Delta}$ exist that do not contain heavy atoms such as rubrene and Buckminster fullerenes. The ISC rates in these chromophores are believed to exist due to the low energy gap between $S_{1}$ and $T_{1}$. Furthermore, using the $\phi_{\Delta}$ to represent the $\phi_{\mathrm{t}}$ is not always an appropriate approximation. The excited state lifetime and solvent effects can result in low $\phi_{\Delta}$ even with a high $\phi_{\mathrm{t}}$. Furthermore, the methods in which $\phi_{\Delta}$ is measured vary and can result in different literature values from the same chromophore.

\subsection{Materials and methods}

\subsubsection{Computational modeling}


All fluorescein derivatives were optimized using B3LYP level of theory with a split basis set treating $\mathrm{C}, \mathrm{H}, \mathrm{O}, \mathrm{Cl}$, and $\mathrm{Br}$ atoms with 6-311 $\mathrm{G}(\mathrm{d})$ and LANL2dz for I atoms. After optimization of the structures an energy calculation was performed using the keywords density $=$ current and $p o p=$ nboread. Heavy atom contributions were determined by feeding the energy calculation into Multiwfn using the natural atomic orbital method as previously described. ${ }^{6}$

\subsubsection{Synthesis of $3^{\prime}, 4^{\prime}, 5^{\prime}, 6^{\prime}$-tetrachloro fluorescein}

3', 4', 5', 6'-tetrachloro fluorescein was synthesized by a solid state reaction previously reported. ${ }^{10}$ Tetrachloro phthalic anhydride $(5.091 \mathrm{~g}, 17.86 \mathrm{mmol})$ was ground with of resorcinol $(3.862 \mathrm{~g}, 35.10 \mathrm{mmol})$ and $\mathrm{ZnCl}_{2}(0.680 \mathrm{~g}, 5.01 \mathrm{mmol})$ as a catalyst. The mixture was then heated in a sandbath $\left(140^{\circ} \mathrm{C}\right)$ under nitrogen flow for 40 minutes. While still warm, $\mathrm{MeOH}$ ( $5 \mathrm{~mL} / 1 \mathrm{mmol}$ of phthalic anhydride) was added and sonicated until all solid was dissolved. Water $\left(15 \mathrm{~mL} \mathrm{H}_{2} \mathrm{O} / 1 \mathrm{mmol}\right.$ phthalic anhydride) was then added and the precipitated product was obtained via vacuum filtration. The filtered product $7.455 \mathrm{~g}, 88 \%$ yield) was then dried by oven $\left(60^{\circ} \mathrm{C}\right)$ overnight.

\subsubsection{Synthesis of FI}

To tetrachlorofluorescein $(0.514 \mathrm{~g}, 1.09 \mathrm{mmol})$ saturated $\mathrm{NaHCO}_{3}(27 \mathrm{~mL})$ and $\mathrm{KI}(15$ $\mathrm{mL}, 0.1 \mathrm{M})$ solution were added. $\mathrm{I}_{2}(0.758 \mathrm{~g}, 2.99 \mathrm{mmol})$ was added and refluxed for 40 minutes then cooled to room temperature. $4 \mathrm{M}$ HCL was added dropwise until a secession of effervescence was observed. Product was obtained via vacuum filtration and

dried for 12 hours in a drying oven. The product was recrystallized in DCM providing of 
FI (0.687 g, 87\% yield). ${ }^{1} \mathrm{H}$ NMR (400 MHz, DMSO-d 6$): 7.02 \mathrm{ppm}(\mathrm{d}, J=14 \mathrm{~Hz}), 6.73$ $\operatorname{ppm}(\mathrm{d}, J=14 \mathrm{~Hz})$.

\subsubsection{Synthesis of FBr}

To tetrabromofluorescein $(0.652 \mathrm{~g}, 1.01 \mathrm{mmol})$ was dissolved in ethanol $(5 \mathrm{~mL})$ in a cool water bath. To this solution 9 eq. of $\mathrm{Br}_{2}$ was added and stirred for 30 minutes. The solution was allowed to sit overnight and the precipitate was isolated by vacuum filtration then dried in an oven over night. The resulting product was pink powder $(0.685 \mathrm{~g}, 73 \%$ yield). ${ }^{1} \mathrm{H}$ NMR (400 MHz, DMSO-d 6$): 7.51 \mathrm{ppm}(\mathrm{s})$.

\subsubsection{General methods for determination of photophysical properties}

$\mathrm{UV}-\mathrm{V}$ is spectra were recorded using a name spectrometer and quartz cuvettes. Stock solutions of $\mathrm{FBr}$ and $\mathrm{FI}\left(10^{-5} \mathrm{M}\right)$ were created in phosphate buffer saline ( $\left.\mathrm{pH} 7.4\right)$. The fluorescence quantum yields were determined using Equation 2.2 with Rose Bengal as the relative standard $\left(\varphi_{\mathrm{f}}=0.016\right)$. The $\phi_{\Delta}$ were determined by the rate of oxygen consumption in DMSO as previously described using Rose Bengal as a standard $\left(\phi_{\Delta}=\right.$ $0.76){ }^{11}$

\subsection{References}

1. Zhao, J., Wu, W., Sun, J. \& Guo, S. Triplet photosensitizers: from molecular design to applications. Chem. Soc. Rev. 42, 5323-5351 (2013).

2. Detty, M. R. \& Merkel, P. B. Chalcogenapyrylium dyes as potential photochemotherapeutic agents. Solution studies of heavy atom effects on triplet yields, quantum efficiencies of singlet oxygen generation, rates of reaction with singlet oxygen, and emission quantum yields. J. Am. Chem. Soc. 112, 3845-3855 (1990). 
3. Rivard, E. Tellurophenes and Their Emergence as Building Blocks for Polymeric and Light-emitting Materials. Chem. Lett. 44, 730-736 (2015).

4. Kryman, M. W. et al. Synthesis and properties of heavy chalcogen analogues of the texas reds and related rhodamines. Organometallics 33, 2628-2640 (2014).

5. Farrell, K. M., Brister, M. M., Pittelkow, M., Sølling, T. I. \& Crespo-Hernández, C. E. Heavy-Atom-Substituted Nucleobases in Photodynamic Applications: Substitution of Sulfur with Selenium in 6-Thioguanine Induces a Remarkable Increase in the Rate of Triplet Decay in 6-Selenoguanine. J. Am. Chem. Soc. 140, 11214-11218 (2018).

6. Acharya, R., Cekli, S., Zeman, C. J., Altamimi, R. M. \& Schanze, K. S. Effect of Selenium Substitution on Intersystem Crossing in $\pi$-Conjugated Donor-AcceptorDonor Chromophores: The LUMO Matters the Most. J. Phys. Chem. Lett. 693697 (2016) doi:10.1021/acs.jpclett.5b02902.

7. Fleming, G. R., Knight, A. W. E., Morris, J. M., Morrison, R. J. S. \& Robinson, G. W. Picosecond Fluorescence Studies of Xanthene Dyes. J. Am. Chem. Soc. 99, 4306-4311 (1977).

8. Wilkinson, F., Ross, A. \& Helman, P. Quantum Yields for the Photosensitized Formation of the Lowest Electronically Excited Singlet State of Molecular Oxygen in Solution. J. Phys. Chem. A 22, 113-256 (1992).

9. Frisch, M. J. et al. Gaussian 09, Revision B.01; Gaussian. Inc.: Wallingford, CT 2009-2009 (2010). doi:111.

10. Pietrancosta, N. et al. Rose Bengal analogs and vesicular glutamate transporters (VGLUTs). Bioorganic Med. Chem. 18, 6922-6933 (2010).

11. Lutkus, L. V., Rickenbach, S. S. \& McCormick, T. M. Singlet oxygen quantum yields determined by oxygen consumption. J. Photochem. Photobiol. A Chem. 378, 131-135 (2019). 


\section{Conversion of Light to Mechanical Energy by Reversible Oxygen Consumption}

\subsection{Background}

Pressure differentials have been used by humans for hundreds of years to produce mechanical or electrical energy. The most common example being that of the internal combustion engine. Upon ignition of hydrocarbons the production of carbon dioxide and water vapor produce a high pressure which is then compensated for by a change in volume by the raising of a piston. The utility of the system depends on the continuous burning of hydrocarbons for the repeated raising and lowering of a piston to perform pressure-volume work (PV work). While PV work has been historically depended on petroleum fuels, the call for emission free sources of energy may limit its future usage.

The search for alternative energies has led to the development of a wide variety of systems to reduce the usage of fossil fuels. A common theme among most alternative energy sources is transforming solar radiation into useful forms of energy. Transforming solar energy to electricity can be directly achieved using photovoltaics such as silicon solar cells. Other approaches have focused on solar to electrical conversion through indirect methods. Photocatalysts have been used to store solar energy in the form of chemical potential in solar fuels such as hydrogen, methanol, and hydrogen peroxide. ${ }^{1-3}$ Photothermal farms transform solar energy indirectly to mechanical energy via conversion of sunlight to thermal energy to produce steam and push a turbine. Few strategies for the direct conversion of light to mechanical energy exist. ${ }^{4}$ The most 
common approach of photo-mechanical conversion is found in photo-actuators which have utilized photothermal effects or photo-isomerization between $E$ and $\mathrm{Z}$ conformations to produce structural or property changes. ${ }^{5-7}$ A prototype light driven engine has been reported which uses the photo-isomerization of an azobenzene compound. Solubility differences between the $E$ and $Z$ conformations were used to produce osmotic pressure to run the engine. ${ }^{7}$ While such technologies are promising, they are limited to specific wavelengths to induce the desired isomerization and often require high energy UV-light.

Herein, we introduce the unusual case of solar to mechanical energy conversion by pairing a light driven oxygen consuming reaction with its dark release of oxygen. The reaction utilizes 1 ,4-dimethyl naphthalene $\left(1,4\right.$-DMN) reacting with singlet oxygen $\left({ }^{1} \mathrm{O}_{2}\right)$ produced by a photosensitizer. The oxygen becomes trapped in the metastable form of an endoperoxide (1,4-DMN EP) resulting in a pressure decrease over time. In a dark reaction, the 1,4-DMN EP releases oxygen to regenerate the 1,4-DMN and results in a pressure increase (Figure 6.1). The results show promise for utilization of such a system for the unique opportunity to convert solar energy to mechanical energy via isothermal PV work. 


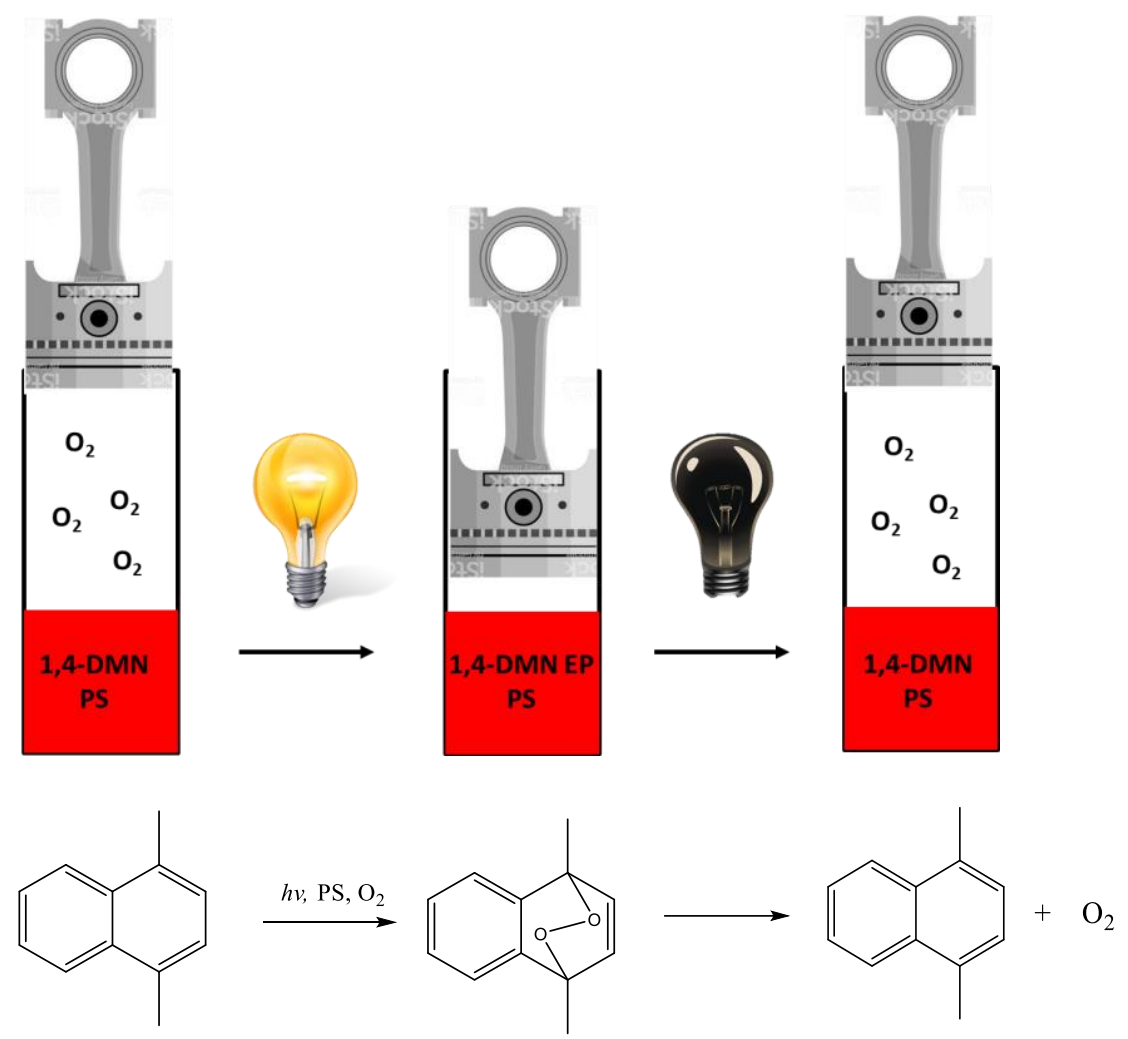

Figure 6.1. Depiction of PV work by oxygen consumption and release using the reversible reaction of $1,4-\mathrm{DMN}$ with ${ }^{1} \mathrm{O}_{2}$.

\subsection{Results and discussion}

\subsubsection{Pressure changes from oxygen consumption and release}

The reaction of ${ }^{1} \mathrm{O}_{2}$ with naphthalene and anthracene compounds to produce their respective endoperoxides has been well studied for cancer therapeutic agents, organic oxidations, and ${ }^{1} \mathrm{O}_{2}$ measurements. The rates of endoperoxide formation, dissociation, and degradation are heavily dependent up on the 1,4 substituents for naphthalene compounds or 9,10 positions in anthracene compounds. The rate of oxidation ultimately relies on the stability of the endoperoxide formed and therefore inversely effects the rate of ${ }^{1} \mathrm{O}_{2}$ release. For example, acyloxy anthracenes are inert to ${ }^{1} \mathrm{O}_{2}, 1,4-\mathrm{DMN}$ forms a metastable endoperoxide at $25^{\circ} \mathrm{C}$ while the 1,2,3,4-tetra substituted derivative forms a 
stable endoperoxide that does not release ${ }^{1} \mathrm{O}_{2}$ at $25^{\circ} \mathrm{C}$. Replacing methyl groups with ether groups for naphthalene compounds such as 1,4-dimethoxy naphthalene can irreversibly react with ${ }^{1} \mathrm{O}_{2}$ to form a quinone via a proposed hydroperoxide intermediate. ${ }^{8}$ For the purposes PV work by the consumption and release of oxygen we chose 1,4-DMN because its metastable endoperoxide allows for the light driven oxidation and dark release of oxygen on a reasonable time scale and at mild temperatures.

To monitor the oxygen consumption and release over time, a ring of LEDs was used to irradiate samples in sealed reaction vessels attached with pressure sensors. The LEDs were controlled by a digital timer to turn the lights on and off at specific time increments. ${ }^{1} \mathrm{O}_{2}$ was produced using Rose Bengal $\left(\phi_{\Delta}=0.76\right)$ as the photosensitizer under green LED irradiation. A chiller was used to implement temperature control to the system.

As shown in Figure 6.2, preliminary results at $25^{\circ} \mathrm{C}$ and atmospheric conditions show a pressure change of approximately 0.5 PSI over the course of 6 hours. Immediately upon turning the lights off a pressure increase was observed. After 6 hours of darkness the pressure nearly returned back to the initial conditions. Repeating the cycle of light on-light off shows nearly an identical response of oxygen consumption and release suggesting minimal degradation to the Rose Bengal or 1,4-DMN. In theory, the consumption of oxygen and release may be cycled in perpetuity because the photosensitizer does not react while the 1,4-DMN and oxygen are regenerated making the system catalytic. Furthermore, it's important to note that a Rose Bengal was selected as 
the photosensitizer because of its high $\phi_{\Delta}$, however, a wide variety of ${ }^{1} \mathrm{O}_{2}$ photosenstizers have been reported and a "cocktail" of photosensitizers may be used to capture the visible to NIR solar spectrum.

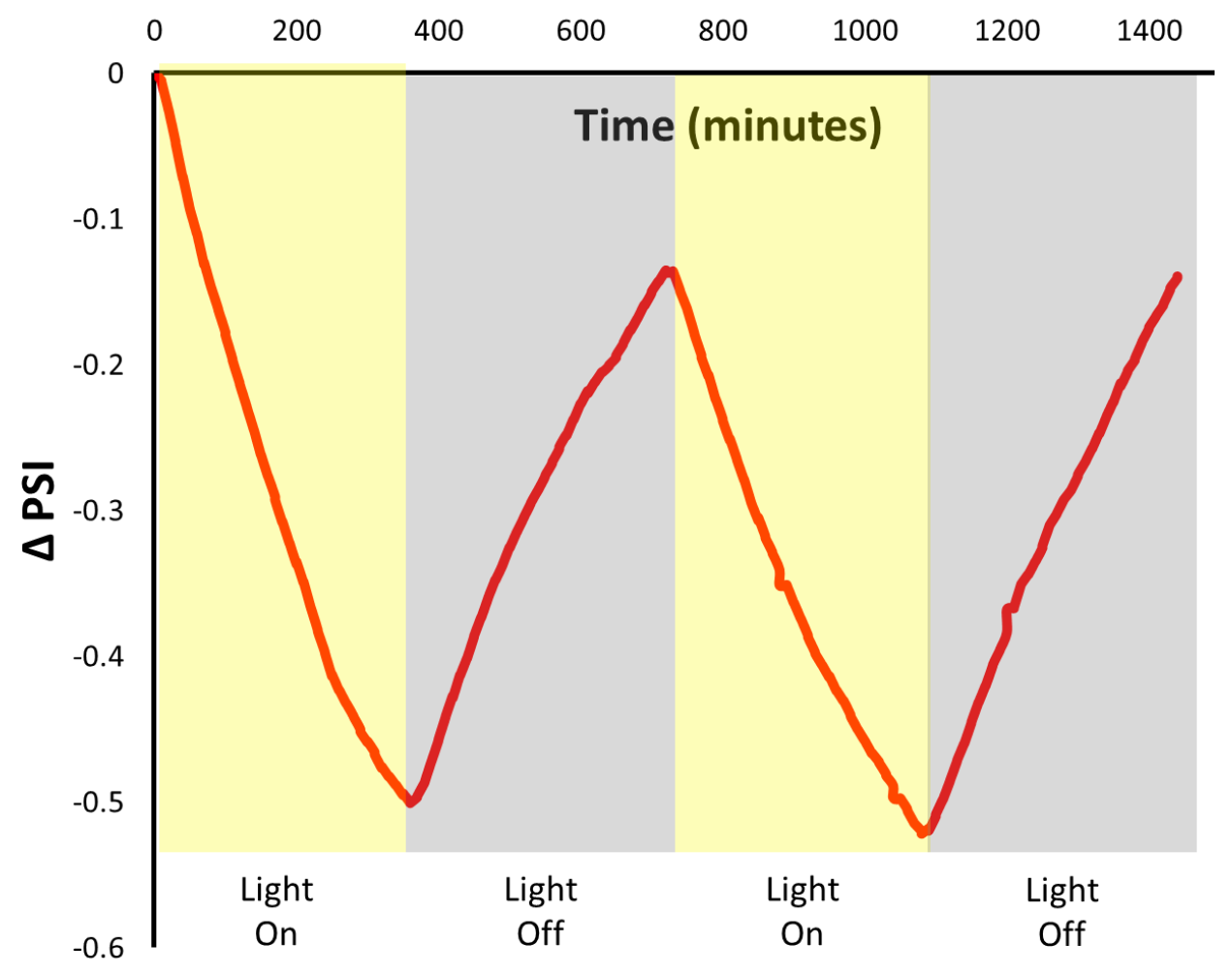

Figure 6.2. Change in pressure over time with intermittent irradiation of 1,4-DMN $(0.064$ M) with Rose Bengal $\left(1 \times 10^{-5}\right)$ under atmospheric pressure at $25^{\circ} \mathrm{C}$.

\subsubsection{Effect of temperature on oxygen consumption and release}

Typically, PV work is accomplished from the thermal expansion and contraction of a gas. The proposed system is unique because it can operate at constant temperature. Ideally, the rates of oxygen consumption could be tailored to fit the daylight hours and 
then release oxygen to fully regenerate over the night. The temperature of the system effects the oxygen consumption in two ways. Increasing the temperature results in destabilization of the 1,4-DMN EP which means the rate of oxygen release will be increased. Furthermore, the increased temperature lowers the amount of dissolved oxygen, further increasing the pressure.

The change in temperature should affect the rate of oxygen release inversely to the consumption since the observed pressure change is a result of the competing forward and back reaction rates. Lower temperatures would stabilize the 1,4-DMN EP resulting in a slower release of oxygen. To explore the effect of temperature, samples of 1,4-DMN with Rose Bengal were irradiated for 6 hours and then in the dark for 6 hours at $20^{\circ} \mathrm{C}, 25$ ${ }^{\circ} \mathrm{C}$, and $30^{\circ} \mathrm{C}$ as shown in Figure 6.3. 


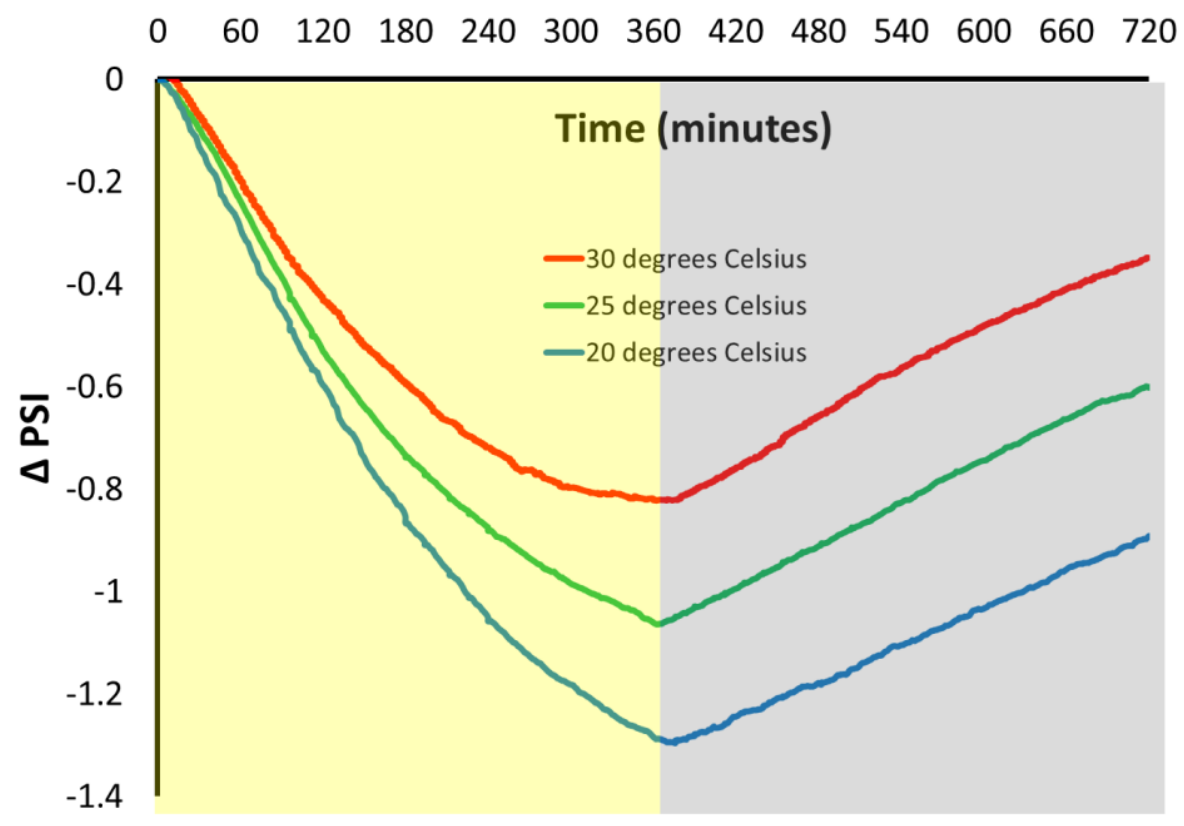

Figure 6.3. Oxygen consumption and release at 1,4-DMN $(0.064 \mathrm{M})$ with Rose Bengal $\left(1 \times 10^{-4} \mathrm{M}\right)$ under atmospheric pressure at $20{ }^{\circ} \mathrm{C}, 25{ }^{\circ} \mathrm{C}, 30^{\circ} \mathrm{C}$.

While at all temperatures the initial rate of oxygen consumption up until 60 minutes is similar, interestingly at $30{ }^{\circ} \mathrm{C}$ the decrease in pressure plateaus around 300 minutes even under continued irradiation. At a pressure decrease of 0.8 PSI approxminately $1 / 8$ of the $1,4-\mathrm{DMN}$ would be oxidized. Complete oxygen consumption from the headspace would result in a pressure change of approximately 3 PSI. Therefore, the plateau of pressure change is indicative of the competing reaction rates reaching equilibrium. A similar trend is observed at 25 and $20{ }^{\circ} \mathrm{C}$ but to a lesser extent. The initial rates of pressure decrease appear linear and the rate of oxidation is dominant, however, over time the rate of oxygen release becomes competitive and results in a slower rate of 
pressure decrease. By irradiating a sample at $25{ }^{\circ} \mathrm{C}$ continuously it was found that equilibrium was reached at a pressure decrease of 1.4 PSI as seen in Figure 6.5.

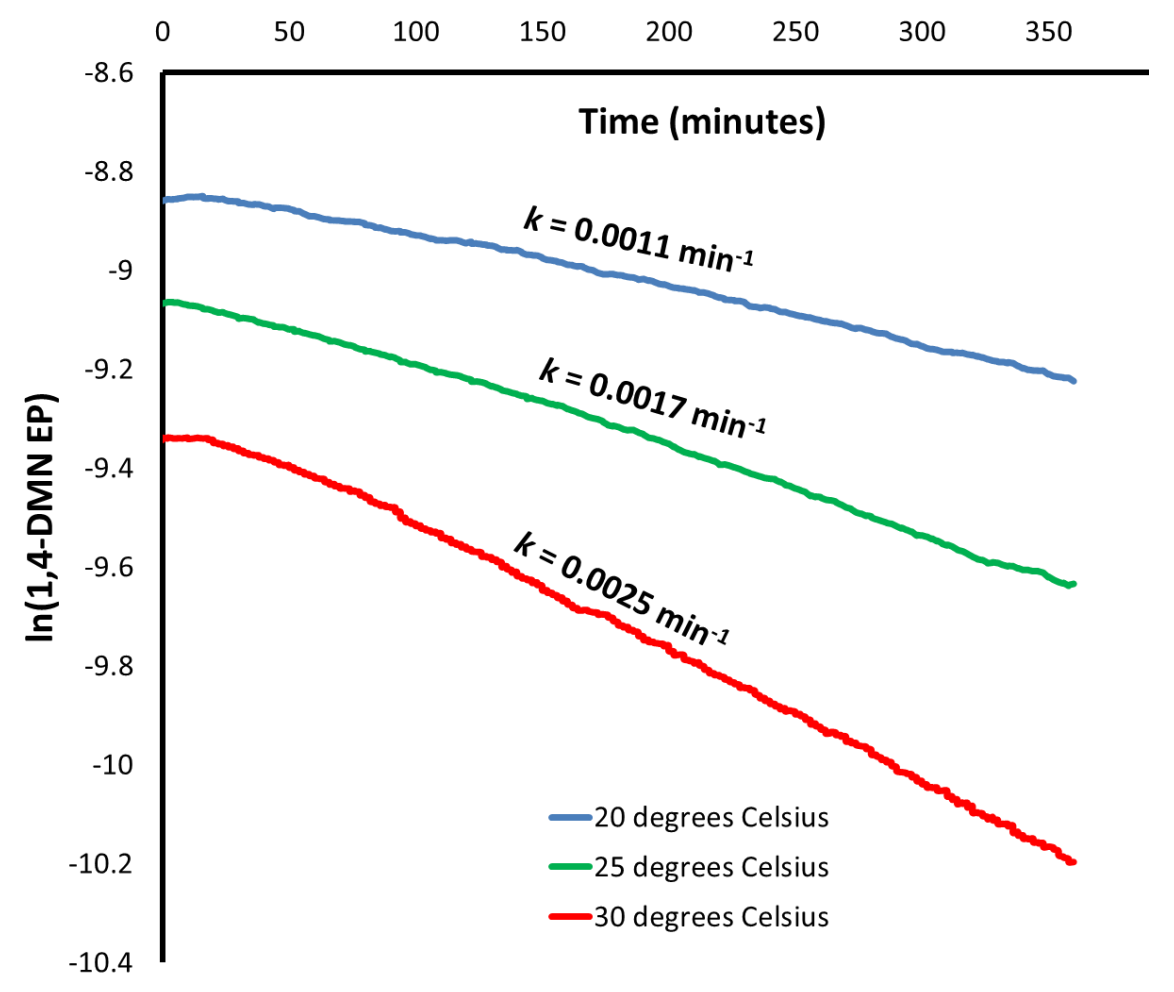

Figure 6.4. Rates of 1,4-DMN EP oxygen release determined by pressure increase at varying temperatures.

As might be expected, the rate of oxygen release in the dark shows an inverse trend. The fastest rate of pressure increase occurs at $30^{\circ} \mathrm{C}$ followed by $25^{\circ} \mathrm{C}$ and $20{ }^{\circ} \mathrm{C}$. At $30{ }^{\circ} \mathrm{C}$ it is evident that the of rate oxygen release slows over time resulting and therefore results in a nonlinear pressure increase. The decomposition of $1,4-\mathrm{DMN}$ is a unimolecular reaction and therefore should follow a first order rate. This would explain the slower rate of pressure increase over time. When plotted as the first order rates of the 
concentration of $1,4-\mathrm{DMN}$ EP the rate of oxygen release at $20{ }^{\circ} \mathrm{C}$ is $0.0011 \mathrm{~min}^{-1}$ which is less than half the rate at $30{ }^{\circ} \mathrm{C}\left(0.0025 \mathrm{~min}^{-1}\right)$.

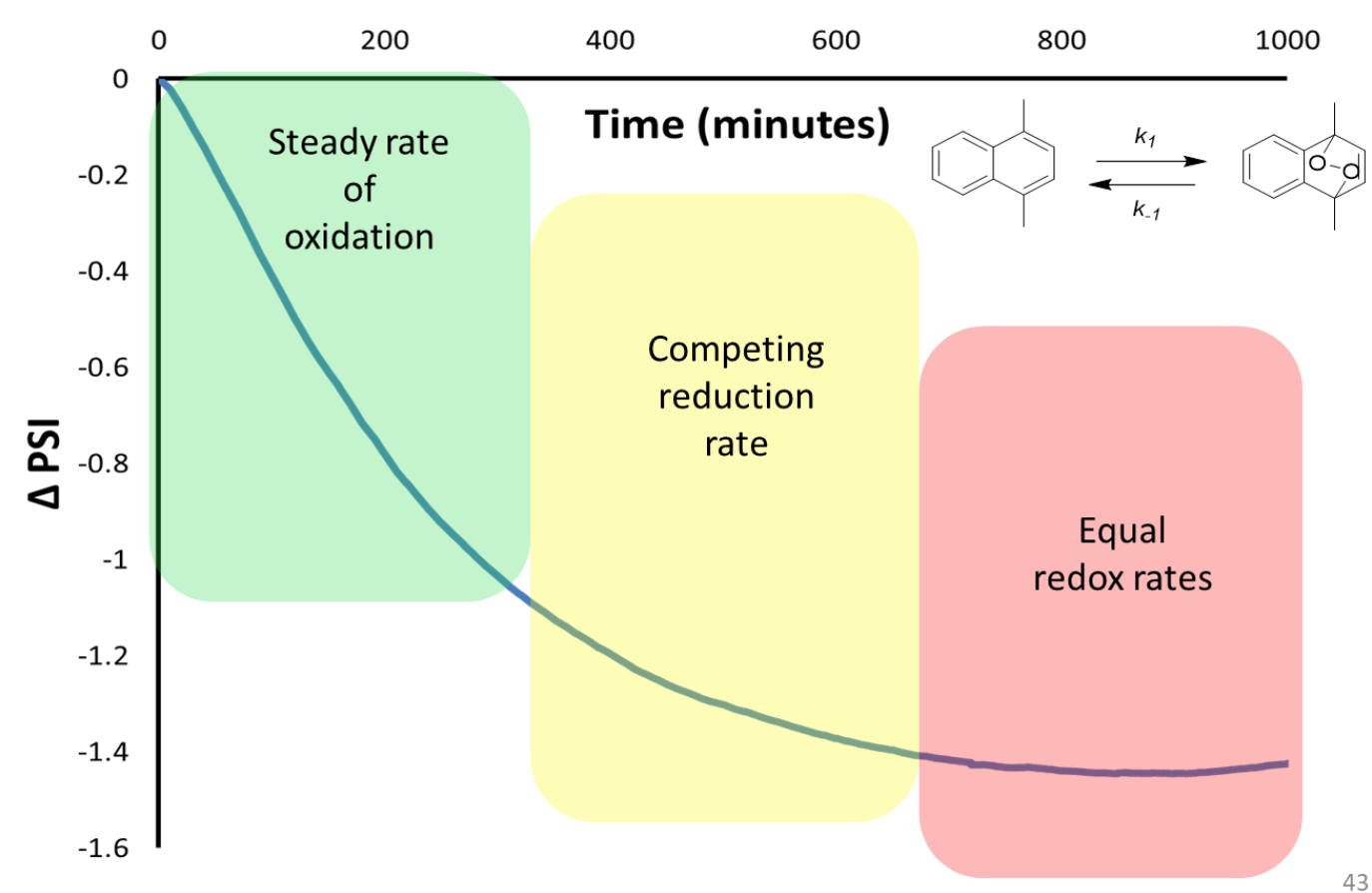

Figure 6.5. Continuous irradiation of Rose Bengal $\left(1 \times 10^{-4} \mathrm{M}\right)$ and 1,4-DMN $(0.064 \mathrm{M})$ under atmospheric conditions at $25^{\circ} \mathrm{C}$ until pressure decrease no longer occurs.

The results show a balance is needed to achieve optimization of the reversible pressure changes to perform PV work. At a constant temperature the rate of oxygen release is fixed, however, the rate of oxidation initially appears dependent on the rate of oxygen dissolution. To assist the oxygen dissolution, the pressure of the vessel was increased by 5.3 PSI compared to atmospheric pressure and continuously irradiated for 
20 hours. As can be seen in Figure 6.6, the increased pressure was able to push the equilibrium to consume more oxygen and therefore create a greater pressure decrease.

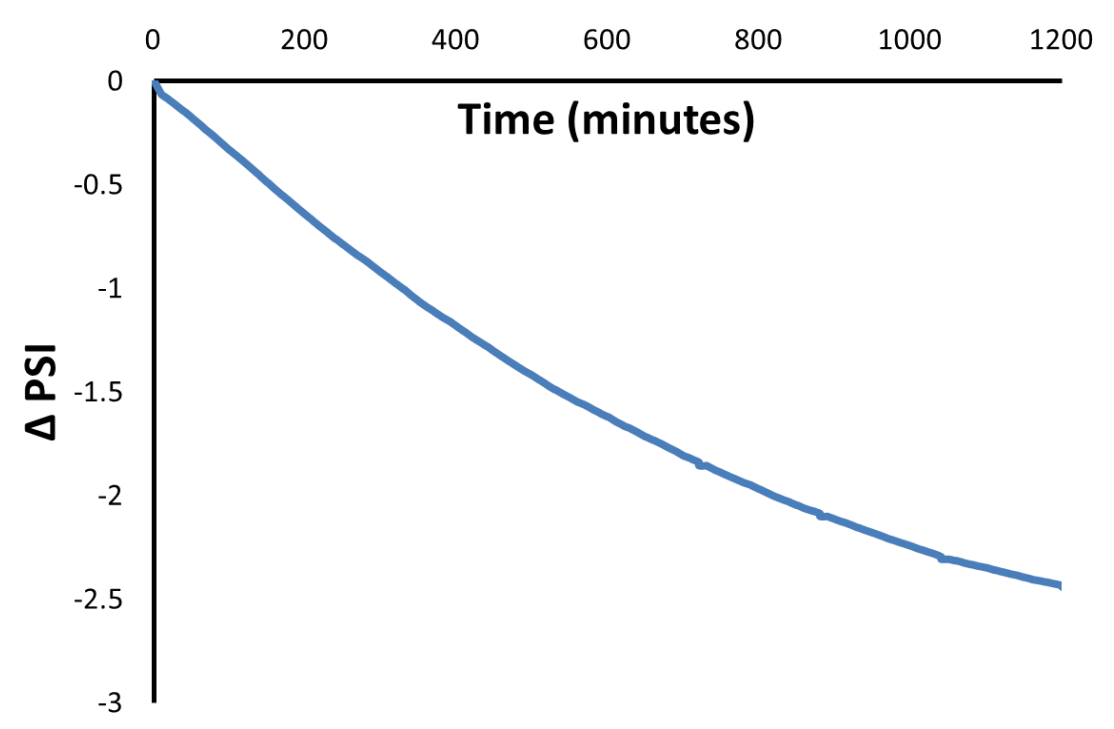

Figure 6.6. Continuous irradiation of Rose Bengal $\left(1 \times 10^{-4} \mathrm{M}\right)$ with 1 ,4-DMN $(0.064$ M) at $25^{\circ} \mathrm{C}$ under an increased pressure of 5.3 PSI compared to atmosphere (20.2 PSI).

The rate of oxidation was also found to increase by replacing the headspace with pure oxygen rather than atmospheric conditions. Replacing the headspace with oxygen increased the rate of oxidation significantly. A rate of pressure decrease of 0.0094 $\mathrm{PSI} / \mathrm{min}$ was achieved as compared to $0.0024 \mathrm{PSI} / \mathrm{min}$ under atmospheric conditions. However, at $25^{\circ} \mathrm{C}$ the only way to compensate for such a fast rate of oxidation is to lengthen the time in the dark to allow oxygen release back to the starting conditions.

\subsubsection{Longevity of oxygen consumption and release}


The proposed system is intriguing because of its catalytic nature. However, its true utility depends on the amount of cycles that may be performed. Inadvertent degradation of the 1,4-DMN over time would limit the life span of the system. To probe the stability of the system a sample with an oxygen atmosphere was put through 4 cycles of irradiation for 90 minutes followed by 3 hours of darkness (Figure 6.7).

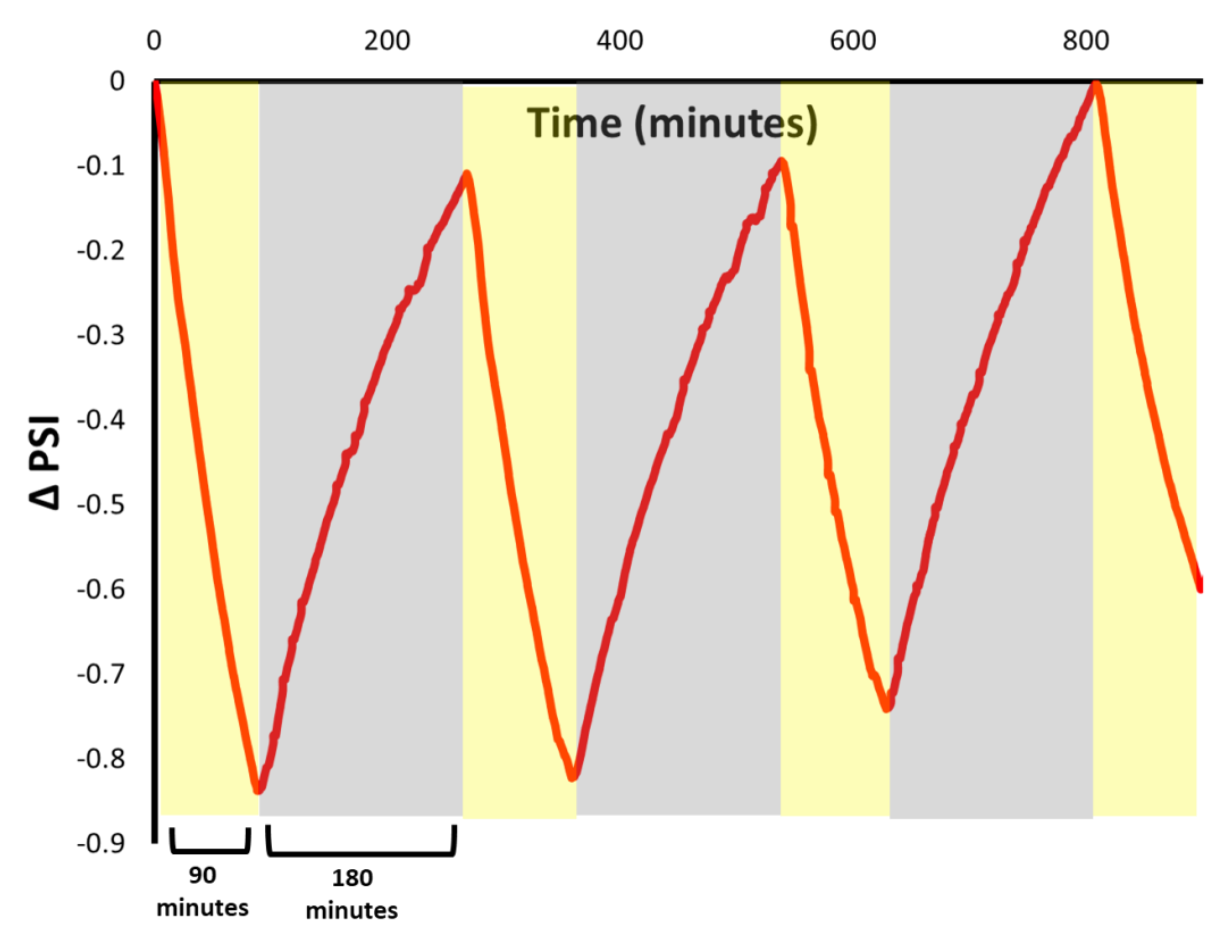

Figure 6.7. Pressure change over time of 3 cycles of irradiation Rose Bengal $\left(1 \times 10^{-4}\right.$ M) with 1,4-DMN $(0.064 \mathrm{M})$ for 90 minutes and 3 hours of darkness at $25^{\circ} \mathrm{C}$ under an oxygen atmosphere.

An apparent change in the rate of oxidation can be seen by the slowing rate of pressure decrease from each cycle of irradiation. Despite this, the similar rates of oxygen release indicate $1,4-\mathrm{DMN}$ is stable. Otherwise, if nonreversible oxidation was occurring 
then the release of oxygen would be slowed. The results suggest that instead photobleaching of Rose Bengal is resulting in a slower rate of oxidation. The bleaching of Rose Bengal would result in less ${ }^{1} \mathrm{O}_{2}$ production and therefore alter the rate of oxidation.

To further probe the source of the depleting rate of oxidation a combination UVVis spectroscopy was used to follow the concentration of Rose Bengal and 1,4-DMN. The absorbance of Rose Bengal and 1,4-DMN was followed at $554 \mathrm{~nm}$ and $289 \mathrm{~nm}$, respectively. Following two cycles of green LED irradiation for 90 minutes and 3.5 hours of darkness the absorbance spectrum indicated a $32 \%$ degradation to the Rose Bengal by the diminishing absorbance at $554 \mathrm{~nm}$. The absorbance of 1,4-DMN was within $5 \%$ of the initial absorbance suggesting the slowed rate of oxidation as previously shown is solely a result of photobleaching from the Rose Bengal.

\subsection{Conclusion}

The transformation of visible light energy to mechanical energy is unique. The presented system utilizes irradiation to excite a triplet-photosensitizer which can be quenched by ${ }^{3} \mathrm{O}_{2}$ to give rise to ${ }^{1} \mathrm{O}_{2}$. The ${ }^{1} \mathrm{O}_{2}$ may then react with $1,4-\mathrm{DMN}$ to produce its corresponding endoperoxide, 1,4-DMN EP, effectively trapping the oxygen in solution. Overtime the oxidation of 1,4-DMN results in the consumption of oxygen and in a closed system results in a pressure decrease. The 1,4-DMN EP is meta-stable near RT and can release the oxygen to return the pressure to the initial conditions while 
regenerating the 1,4-DMN. By cycling the irradiation on and off the system shows the capability of repeated cycles to preform PV-work.

The presented system shows a number of unique advantages compared to many other solar energy systems. Since the role of the triplet photosensitizer is only to produce ${ }^{1} \mathrm{O}_{2}$, a wide variety of dyes could be used to absorb across the visible spectrum including NIR light. However, from an energetic perspective this system is unique in the fact that efficiency increases at lower energy irradiation because each photon absorbed is used for ultimately the same goal of producing 1,4-DMN EP. Furthermore, the reverse reaction does not require light to release oxygen and therefore such a system could be used at night time which is a major challenge for most solar energy technologies. While photothermal, techniques have been used to transform solar energy into thermal energy, this system can be cycled at a constant temperature. Future research will focus on transforming this system to the solid state to avoid the necessity of oxygen dissolution.

\subsection{Materials and Methods}

\subsubsection{Chemicals used}

1,4-DMN of a purity $>99 \%$ was graciously donated by the 1,4-Group. All reactions were carried out in acetonitrile (HPLC grade) with Rose Bengal from Sigma Aldrich.

\subsubsection{General set up for monitoring change of pressure over time}

The change in pressure over time were monitored using sealed $50 \mathrm{~mL}$ reaction vessels with Omega gage pressure sensors to monitor the pressure every two minutes 
using the TracerDAQ Stripchart program. Green LEDs lining a steal tube were used to evenly irradiate up to twelve samples held in a Radleys Carousel 12 with an electronic timer to turn the lights on or off at determined time increments. The solutions were stirred at 500 RPM and a chiller was wrapped around the LED ring to control the temperature. The entire apparatus was covered to avoid inadvertent irradiation and irradiation was not initiated until temperature equilibration occurred as indicated by a plateau in pressure change. The headspace of the reaction vessel was either left as atmosphere or replaced with oxygen by purging with under vacuum or with pure oxygen three times and allowed to regain atmospheric pressure.

\subsection{References}

1. Fukuzumi, S. Artificial photosynthesis for production of hydrogen peroxide and its fuel cells. Biochim. Biophys. Acta (2015) doi:10.1016/j.bbabio.2015.08.012.

2. McCormick, T. M. et al. Reductive side of water splitting in artificial photosynthesis: new homogeneous photosystems of great activity and mechanistic insight. J. Am. Chem. Soc. 132, 15480-3 (2010).

3. Liu, H. et al. A review of anode catalysis in the direct methanol fuel cell. J. Power Sources 155, 95-110 (2006).

4. Baumgartner, B. Process for Converting Light to Mechanical Power. (1962).

5. Kim, D., Lee, H. S. \& Yoon, J. Highly bendable bilayer-type photo-actuators comprising of reduced graphene oxide dispersed in hydrogels. Sci. Rep. 6, 1-10 (2016).

6. Lee, E., Kim, D., Kim, H. \& Yoon, J. Photothermally driven fast responding photo-actuators fabricated with comb-type hydrogels and magnetite nanoparticles. Sci. Rep. 5, 1-8 (2015).

7. Masiero, S., Lena, S., Pieraccini, S. \& Spada, G. P. The direct conversion of light into continuous mechanical energy by photoreversible self-assembly: A prototype of a light-powered engine. Angew. Chemie - Int. Ed. 47, 3184-3187 (2008). 
8. Bauch, M., Krtitschka, A. \& Linker, T. Photooxygenation of oxygen-substituted naphthalenes. J. Phys. Org. Chem. 30, 1-11 (2017). 


\section{Conclusions}

The absorbance of light by molecules and the subsequent processes that occur are of great importance to develop future alternative energy sources and synthetic approaches. The integral measurement for the feasibility and widespread adoption of such processes is its quantum yield. This work focuses specifically on the importance of quantum yield with respect to both experimental design in its determination and it as a measure of efficiency for different photochemical processes.

While quantum yield is a broad term that may be used to describe a variety of photoinduced processes, it is typically measured using spectroscopic methods. This work has described simplified methods for the determination of singlet oxygen quantum yields as well as the photoreduction of potassium ferrioxalate. Both of the developed methods rely on monitoring the change of pressure over time. Singlet oxygen quantum yields were determined by the pressure decrease created by oxygen consumption though photochemical production of singlet oxygen that reacts with DMSO. Additionally, a new method for chemical actinometry using potassium ferrioxalate was developed by following the pressure increase over time from the production of carbon dioxide. The two methods avoid the need for spectroscopic monitoring to determine reaction kinetics. As such, this allows for both reactions to be monitored in real-time rather than gathering data points from aliquots at designated time periods, using expensive spectrometers that may not be available in all labs. While both methods were developed using a specialized photo-reactor with pressure sensors, a considerable effort was made to adjust both 
methods to use standard lab equipment for widespread implementation. This is specifically significant to standardize a method for the determination of quantum yields for precisely reported values.

While a wide variety of experiments have been developed for quantum yield measurements, all require either specialized chemicals or instrumentation. Some methods have proved more popular than others, however, a wide variety of methodologies are still reported. This may be, at least in part, why multiple different quantum yields are reported for the same systems. While such measurements have inherent difficulties due to the large number of photons absorbed per second and susceptibility of such systems to stray light, a normalized approach could help produce more consistent reported quantum yields.

The simplification of these methods may prove beneficial for the comparison of photocatalysts. Both methods described are comparably less labor intensive than existing traditional methods which can allow for faster screening of photocatalysts and optimization of light driven systems. Furthermore, they both benefit from the ability to collect rates at real time. This is important because it eliminates the need to remove the sample from the light source. The prevalence of stray light can be difficult to avoid while moving samples in order to complete such measurements. While both methods were carried out using a 12-well photoreactor with pressure sensors, it was also shown that standard lab equipment can be used. This results in an economic advantage which may allow widespread adoption of these newly developed methods. Future work may be 
focused on expanding these methods to show their accuracy for quantum yield determinations across multiple light driven systems.

Many different types of molecules have been developed for the absorption of light to initiate different desired processes, this work has placed a specific focus on tripletphotosensitizers. Fluorescein derivatives and a tellurorhodamine dye were used as tripletphotosenstizers under aerobic conditions to produce singlet oxygen. Both types of dyes incorporate heavy atoms in their structures to elicit the heavy atom effect. A focus on a quantitative understanding of the heavy atom effect in relation to the singlet oxygen quantum yield has been discussed. Fluorescein derivatives with heavy atoms ( $\mathrm{Br}$ or I) were computationally modelled to determine the heavy atom's contribution to frontier molecular orbitals. The results show that fluorescein derivatives with higher contributions from heavy atoms to the LUMO tend to have higher singlet oxygen yields. This may also be a plausible explanation as to the difference in photophysical properties of other heavy atom containing compounds such as tellurium containing chromophores. The tellurorhodamine studied has a high singlet oxygen yield, however, this is diminished upon oxidation to produce the telluroxide. While the telluroxide still contains a heavy atom $(\mathrm{Te})$ it is fluorescent with a low singlet oxygen quantum yield. Computationally modeling the percent contribution to the LUMO shows a large drop in contribution from the tellurium atom to the LUMO for the telluroxide. These results are in agreement with the trend among fluorescein derivatives and their respective heavy atom contributions. 
The ability to predict the quantum yields for population the triplet-excited-state is particularly significant for the development of more efficient photocatalysts. There are often many competitive relaxation pathways a chromophore can undergo which makes it difficult to predict how structural changes will effect triplet yields. While predictive modeling of excited-state chromophores can be dangerous in terms of over simplifying complex systems, the benefits well outweigh the risks. Having predictive models, for quantum yields of photocatalysts, would significantly ease synthetic labor. Often many derivatives of photocatalysts are developed in the search increased efficiency. Future research could look to utilize computational modeling to predict these properties in advance and could allow for faster progress of developing useful photocatalytic systems.

The model, in theory, could be used to either optimize the triplet yield or minimalize it in cases where a triplet state is not desired. While this study was limited to fluorescein derivatives this method may also find utilization for other classes of chromophores to help predict the properties of other derivatives. Future research should look to expand this model as experimental data on more derivatives becomes available. Additional factors, such as both singlet and triplet energy levels, could be included in future models to increase the accuracy of the predictive power for more complex systems.

Known triplet-photosensitizers were used in photochemical systems for conversion of light for potential use for photocatalysis and as an alternative energy source. A photocatalytic system using a unique tellurium-containing triplet-photosensitizer was explored for photocatalysis. While heavy atoms are often incorporated into the structure 
of triplet-photosensitizers typically halogens are used. Incorporation of tellurium as the heavy atom in rhodamine derivatives was used because of its unique reactivity. The tellurorhodamine used can create singlet oxygen under irradiation and also react with singlet oxygen at the tellurium heteroatom. The reaction of singlet oxygen with the tellurium heteroatom produced a telluroxide which was shown to act as an oxidant for the conversion of thiols to disulfides.

This is work is of particular significance because it demonstrates the utility of the reaction of the tellurium heteroatom with singlet oxygen to produce a telluroxide and thus overcomes the limitations of the transient nature singlet oxygen as an oxidant. The system allows for the catalytic oxidation of thiol substrates requiring only light and aerobic conditions. In future research, a similar photocatalytic process could be developed for other oxidations and would only require light and atmospheric air. Therefore, avoiding waste from stoichiometric reagents, byproducts, and high temperature or pressure conditions. Future research could use this system for other types of oxidations instead of thiols. The development of similar tellurium containing photocatalysts with structural modifications may be necessary to alter the redox potential to expand the substrate scope of oxidation reactions.

Another photochemical application utilizing triplet-photosensitizers described in this work is the use of the reaction of singlet oxygen with 1,4-dimethyl naphthalene. Similar to results observed from the oxidation of DMSO by singlet oxygen; upon irradiation of Rose Bengel in a closed system the pressure decreased overtime due to 
oxygen consumption. However, unlike the oxidation of DMSO this reaction is reversible near room temperature. In the absence of light the oxidized 1,4-dimethyl naphthalene will release oxygen to regenerate the 1,4-dimethylnapthalene resulting in a corresponding pressure increase. This cycle was repeated multiple times and showed the potential to produce pressure/volume work requiring only sunlight to operate. To our knowledge this is the first example of mechanical energy produced from visible light without using a photothermal effect.

While the results described are all at a constant temperature, the system may be further optimized by increasing the temperature for faster oxygen release or decreasing the temperature for to enhance the endoperoxide formation. Future research should examine the practicality of using such a system as an alternative energy source. Most likely, large areas of land would be needed similar to photothermal farms, however, such a facility would not be confined to only warm climates as photothermal farms require. Different derivatives of naphthalene could be used to optimize the rates of oxygen consumption and release dependent on the temperature as dictated by the climate or season. In theory the entire visible spectrum could be captured using different tripletphotosensitizers. However, more research is needed to limit photobleaching and examine possible side reactions among different photosensitizers.

While it is suggested such a system could convert light to PV work, many other applications exist. Specifically, oxygen storage is of great interest for medicinal, recreational, and emergency use. Theoretically the system could store $1.5 \mathrm{~L}$ of oxygen at 
STP using only $10 \mathrm{~mL}$ of 1,4-dimethyl naphthalene. The reversibility of the system could provide a reusable oxygen storage container that can selectively capture oxygen requiring only light to operate.

While the described work involves five unique projects, they are all intertwined by the common theme of photochemistry. Photochemistry, at its core, is the transformation of light energy. This work shows a wide variety of transformations. Light was used to initiate chemical reactions such as the oxidation of thiols to disulfides and the photoreduction of potassium ferrioxalate. Light was also converted to a pressure change by using a reversible oxygen consuming reaction. A focus was placed on triplet photosensitizers because of their ability to transfer energy from light to excite ground state oxygen to the more reactive singlet oxygen. It was shown that the influence of the heavy atom effect could be used quantitatively to predict the efficiency of singlet oxygen production. Triplet-photosensitizers were used to produce singlet oxygen for aerobic oxidation processes that represent the potential of such photocatalytic systems for future use in sustainable synthesis practices and alternative energy. The new methods developed for determination of both singlet oxygen quantum yields and for actinometry measurements will also facilitate discoveries in this area by providing a similar method for making these critical measurements. 